\title{
Beiträge zur Theorie Abel'scher Gruppen und ihrer Anwendung auf die Zahlentheorie.
}

Von K. Zsigmondy in Wien.

Bei den Untersuchungen über Abel'sche Gruppen, welche den Gegenstand der vorliegenden Arbeit bilden, findet öfters ein besonderes, dem Sieb des Eratosthenes nachgebildetes Verfahren - die Methode des Ausscheidens und Hinzufïgens - mit Vortheil Verwendung. Um jedoch den Zusammenhang der Untersuchungen nicht durch eine Erläuterung dieser Methode stören zu müssen, möge eine kurze Auseinandersetzung derselben nebst einigen ihre Bedeutung charakterisierenden Anwendungen dem eigentlichen Gegenstande dieser Arbeit vorangehen.

Was diesen selbst betrifft, werden zunächst A bel'sche Gruppen, deren Grad eine Primzahlpotenz ist, in Betracht gezogen und für diese die Existenz eines Basissystemes nachgewiesen. Hieran schließt sich naturgemäß die Bestimmung der Anzahl der möglichen Basissysteme einer solchen Abel'schen Gruppe auf Grund der Thatsache, dass die Gradzahlen der Individuen eines jeden derartigen Systemes und die Anzahl der Basiselemente eines jeden Grades unabhängig ron der Wahl des Basissystemes sind. Die zur Darlegung dieser Resultate durchgeführten Betrachtungen ermöglichen zugleich einen Einblick in den Auf bau der Untergruppen von einem gegebenen Grade und gestatten, die Anzahl derselben von einer bestimmten "Klasse" zu ermitteln.

Die gewonnenen Sätze können nunmehr leicht auf Abel'sche Gruppen von beliebig zusammengesetztem Grade übertragen werden, weil sich diese stets aus Abel'schen Gruppen, deren Grade Primzahlpotenzen sind, zusammensetzen lassen. Insbesondere wird die Anzahl der regulären Untergruppen eines gegebenen Grades bestimmt, eine Anzahl, welche im weiteren Verlaufe der Untersuchungen noch auf anderem Wege in doppelter Weise ermittelt wird und $\mathrm{zu}$ einer weitgehenden Verallgemeinerung der zahlentheoretischen Function $\varphi(m)$ Veranlassung gibt.

Schon bei den erwähnten Betrachtungen sind zwei Arten von Untergruppen aufgetreten:

1. Der Complex der Wurzeln der Gleichung

$$
x^{\delta}=1 \text {. }
$$

2. Die Gesammtheit der $\delta^{\text {ten }}$ Potenzen. 
Es werden die wichtigsten Eigenschaften beider Gruppengattungen, welche eine vollständige Analogie miteinander zeigen, entwickelt, und es wird insbesondere angegeben, welche Gruppen der $2^{\text {ten }}$ Art zugleich auch der ersten angehören.

Nachdem noch eine dritte Art von Untergruppen, die Multiplicatorengruppen, einer näheren. Betrachtung unterzogen worden sind, werden die allgemeinen Untersuchungen über A bel'sche Gruppen durch eine Reihe von Sätzen abgeschlossen, welche sich auf das aus den Elementen einer A bel'schen Gruppe gebildete System von Combinationen $\mathrm{zu}$ einer bestimmten Klasse beziehen.

Zur Veranschaulichung der entwickelten Theoreme dient zunächst das vollständige Restsystem modulo $m$, das eine Abel'sche Gruppe bezüglich der Addition darstellt, ferner das vollständige mit $m$ theilertremde Restsystem, welches wiederum eine Gruppe bezüglich der Multiplication constituiert. Zum Schlusse folgen einige Betrachtungen über die $\delta^{\text {ten }}$ Potenzreste.

\section{Abschnitt: Vorbemerkungen.}

\section{Nr. 1. Über die Methode des Ausscheidens und Hinzufügens.}

Alle Aufgaben, bei deren Lösung die bekannte zahlentheoretische Function $\mu(x)$ zur Verwendung gelangt, führen, so viel mir bekannt, in allgemeinster Fassung auf das folgende Problem:

"Ein System $T$ von beliebigen, aber wohldefinierten Objecten (Elementen), deren Anzahl auch unbegrenzt sein kann, sei gegeben; terner mögen in $T$ enthaltene Untersysteme erster Art $T_{i}$ $(i=1,2,3, \ldots)$ in der Weise definiert sein, dass, falls unendlich viele solcher Systeme vorhanden sind, jedes Object sich nur in einer endlichen Anzahl von ihnen vorfinde, und endlich möge der Inbegriff der gleichzeitig ${ }^{r}$ beliebigen dieser Systeme $T_{i_{1}}, T_{i_{2}}, \ldots, T_{i_{r}}$ angehörigen Elemente als Untersystem $r^{\text {tex }}$ Art mit dem Symbol $T_{i_{1}, i_{2}, \ldots, i_{r}}$ bezeichnet werden, wobei der einfacheren Darstellung willen auch der Fall vicht ausgeschlossen sei, dass die Systeme $T_{i}, \ldots, T_{i_{r}}$ kein gemeinsames Element besitzen, dass also das Untersystem $T_{i_{1}, \ldots, i_{r}}$ überhaupt kein Object enthält.

Es soll die Gesammtheit derjenigen Objecte des Systemes $T$, welche in keinem der Untersysteme $T_{i}$ (1) $(i=1,2, \ldots)$ enthalten sind, mit Hilfe des ursprünglichen Systemes und der Untersysteme $1_{0}^{\text {tex }}$ und höherer Art dargestellt werden."

Die Lösung dieses Problemes lässt sich ohne Schwierigkeit mittelst der Methode des Ausscheidens und Hinzufügens bewerkstelligen, wie ich das im Folgenden auseinandergesetzte Verfahren nennen will, welches einer bereits im Alterthume unter 
dem Namen "Sieb des Eratosthenes" zur Gewinnung von Primzahlen benützten Methode nachgebildet erscheint.

Man scheide zunächst aus dem Complexe $T$ die Elemente von $T_{1}$ aus. Die Gesammtheit der übrig bleibenden Objecte möge mit

$$
T \cdot T_{1}
$$

bezeichnet werden, wobei der Punkt über dem Subtractions-, be. ziehungsweise Additionszeichen hier, wie im Folgenden, daran erinnern soll, dass man es nicht mit arithmetischen Operationen im gewöhnlichen Sinne zu thun hat.

Aus dem Complexe $T-T_{1}$ sind hierauf diejenigen Objecte von $T_{2}$ auszuscheiden, welche nicht schon mit $T_{1}$ zugleich entfernt worden sind, d. h., da die Gesammtheit der diesen beiden Untersystemen gemeinsamen Objecte mit $T_{1,2}$ bezeichnet worden ist, die Elemente des Systemes $T_{2}^{r} \dot{-} T_{1,2}$. Als den Inbegriff derjenigen Objecte von $T$, welche weder in $T_{1}$, noch in $T_{2}$ enthalten sind, erhält man mithin den Complex

$$
T \div T_{1}-T_{2} \dot{+} T_{1,2}
$$

Aus diesem Systeme hat man nun weiters diejenigen Elemente des Untersystemes $T_{3}$, welche sich weder in $T_{1}$, noch in $T_{2}$ vorfinden, oder nach dem eben gewonnenen Resultate den Complex $T_{3}-T_{1,3}-T_{2,3} \dot{+} T_{1,2,3}$ auszuscheiden und bekommt somit das System

$$
T \cdot T_{1}-T_{2} \div T_{3} \doteqdot T_{1,2} \doteqdot T_{1,3} \doteqdot T_{2,3} \circ T_{1,2,3} .
$$

Setzt man dieses Verfahren fort, so ergibt sich schlieflich der Complex

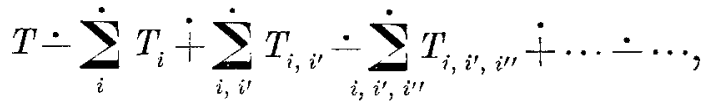

wo die Summen sich auf alle verschiedenen Untersysteme $1^{\text {ter }}$ und höherer Art zu beziehen haben.

Das System (I) enthält kein Object mehr der gegebenen Untersysteme $T_{i}(i=1,2,3, \ldots)$ und stellt somit die Lösung des Problemes (1) dar.

In der That findet sich das Element $A$ von $T$ nach Voraussetzung nur in einer endlichen Anzahl von Untersystemen $1^{\text {ter }}$ Art vor; es seien dies genau die Untersysteme $T_{i_{1}}, T_{i_{2}}, \ldots, T_{i_{2}}(r>0)$. Es tritt dann das Object $A$ in denjenigen und nur in denjenigen Untersystemen höherer Art $T_{j_{1}}, \ldots, j$, natürlich immer nur einmal auf, bei welchen das Indexsystem $j_{1}, \ldots, j_{s}$ irgend eine aus den Zahlen der Reihe $i_{1}, \ldots, i_{r}$ gebildete Combination vorstellt. In 
dem Complexe (I) kommt also das Element $A$ entsprechend den Summen

$$
1-\left(\begin{array}{l}
r \\
1
\end{array}\right)+\left(\begin{array}{l}
r \\
2
\end{array}\right)-\cdots=(1-1)^{r} \mathrm{mal}
$$

d. h. ïberhaupt nicht vor.

Auf Grund der Form des Complexes (I) lässt sich die Methode des Ausscheidens und Hinzufügens durch die folgende Regel charakterisieren:

Um die Gesammtheit derjenigen Objecte des Systemes $T$, welche in keinem der gegebenen Untersysteme $T_{i}(i=1,2,3, \ldots)$ enthalten sind, zu er(2) langen, hat man zu dem Systeme $T$ alle Untersysteme geradzahliger Art hinzuzufügen und hieraufaus dem so gewonnenen Complexe alle Untersysteme ungeradzahliger Art auszuscheiden.

\section{Nr. 2. Einige Anwendungen der Methode des Ausscheidens und} Hinzufügens.

Die Methode des Ausscheidens und Hinzufügens wird im Laufe der folgenden Untersuchungen vielfach Verwendung finden. Um jedoch ihre Bedeutung auch für die Zablentheorie im Allgemeinen darzulegen, sei es gestattet, einige dem zu behandelnden Gegenstande ferne liegende Anwendungen derselben, die zum Theile Erweiterungen bekannter Resultate ergeben werden, in dieser Nummer anzuführen.

Der Kürze halber soll, falls kein Irrthum unterlaufen kann, bei den in der Folge auftretenden Ausdrücken der Form (I) in Nr. 1 die Erklärung der Summenzeichen, welche sich auf die Combinationen zur $1^{\text {ten }}$, oder $2^{\text {ten }}$, oder $3^{\text {ten }}$ Klasse u. s. f. gebildet aus den die Untersysteme charakterisierenden Zahlen zu erstrecken haben, als bekannt, unterdrückt werden. Ebenso wird, um die Bildungsweise der erwähnten Ausdrücke stets deutlich hervortreten zu lassen, von der Einführung der Function $\mu(x)$ oder einer ihr ähnlichen Abstand genommen werden.

Wohl die wichtigste Specialisierung des allgemeinen Problemes (1) der Nr. 1 ist die, dass das System $T$ eine, wenn auch unendliche Gruppe ${ }^{1}$ ) von Elementen hinsichtlich gewisser Operationen bildet, und dass die Systeme $T_{1}, T_{2}, \ldots$ Untergruppen von $T$ darstellen. Dann ist natürlich von selbst das Untersystem $p^{\text {ter }}$ Art $T_{i_{1}, \ldots, i_{r}}$ die größte simultan in den Gruppen $T_{i_{1}}, \ldots, T_{i_{-}}$enthaltene Untergruppe, also der sogenannte größte gemeinsame Divisor von $T_{i}, \ldots, T_{i_{r}}$

1) Bezüglich der Definition einer Gruppe vergl. man Nr. 1 des folgenden. Abschnittes. 
Als erstes Beispiel hierfür möge das folgende dienen:

Es soll der Complex $C$ der positiven ganzen Zahlen $\alpha$, die nicht durch die beliebig angenommenen Zahlen $n_{1}, n_{2}, \ldots, n_{s}$ theilbar sind, angegeben werden.

In diesem Falle hat man für $T$ die Gesammtheit der positiven ganzen Zahlen, welche binsichtlich der Addition und Multiplication eine Gruppe bildet, für die Systeme $T_{i}(i=1,2, \ldots, s)$ jedoch die Untergruppen $n_{i} k(k=1,2,3, \ldots)$ zu setzen. Von selbst stellt sich dann der größte gemeinsame Divisor $T_{i_{1}, \ldots, i_{r}}$ durch die Gruppe $\dot{n}_{i_{1}, \ldots, i_{r}} k(k=1,2,3, \ldots)$, in welcher die Zahl $n_{i_{1}, \ldots, i_{r}}$ das kleinste gemeinsame Vielfache der Zahlen $n_{i}, \ldots, n_{i}$ bedeutet, dar und der gesuchte Complex $C$ wird entsprechend der Form (I) in Nr. 1 durch die Gruppen $n_{i_{1}, \ldots, i_{\mathrm{r}}} k(k=1,2,3, \ldots)$ ausgedrückt erscheinen.

Bezeichnet man also mit $f(x)$ eine willkürliche Function aller ganzen Zahlen $x$, welche bloß die Eigenschaft besitzt, dass die Reihe $\sum_{j=1}^{\infty}|f(j)|$ convergiert, so gelangt man zu der Gleichung

$$
\sum_{\alpha} f(\alpha)=\sum_{j=1}^{\infty} f(j)-\sum_{i} \sum_{j=1}^{\infty} f\left(j n_{i}\right)+\sum_{i, i^{\prime}} \sum_{j=1}^{\infty} f\left(j n_{i, i^{\prime}}\right)-\cdots
$$

Zählt man auf beiden Seiten bloß die bis zum Argumentswerte $m$ auftretenden Glieder, oder mit anderen Worten, setzt man $f(i)$ gleich 0 oder 1 , jenachdem $i$ gröber als $m$ ist, oder nicht, so ergibt sich für die Anzahl der Zahlen, die nicht größer als $m$ und durch keine der Zahlen $n_{1}, \ldots, n_{s}$ theilbar sind, der Ausdruck

$$
m-\sum_{i}\left[\frac{m}{n_{i}}\right]+\sum_{i, i^{\prime}}\left[\frac{m}{n_{i, i^{\prime}}}\right]-\cdots,
$$

welcher, sobald die Zahlen $n_{1}, \ldots, n_{s}$ speciell als theilertremd vorausgesetzt werden, eine bekannte Gestalt annimmt. ${ }^{1}$ )

$\left.{ }^{1}\right)$ Vergl. zar Verallgemeinerung der Function $\varphi(m)$ in der Zahlentheorie. Journ. f. r. u. a. Mathem. Bd. 111.

Bekanntlich hat der i. T. angeführte Ausdruck (I) dadurch auf ein besonderes Interesse Anspruch, dass er im Falle, als für $n_{1}, \ldots, n_{s}$ die $s$ ersten Primzahlen $p_{1}, \ldots, p_{s}$ gesetzt werden, für jeden Wert $m$, der zwischen $p_{s}$ und $p_{s+1}^{2}$ liegt, die um 1 vermehrte Anzahl der Primzahlen zwischen $p_{s}$ und $m$ durch $m$ und die $s$ ersten Primzahlen dargestellt liefert. Substituiert man nun in dem Ausdrucke (I) für die gröbte ganze unterhalb ron $\frac{m}{p_{i_{1}} p_{i_{2}} \cdots}$ liegende Zahl $\left[\frac{m}{p_{i_{1}} p_{i_{2}} \cdots}\right]$ die nächste ganze $\left\{\frac{m}{p_{i_{1}} p_{i_{2}} \cdots}\right\}$, so gibt der auf diese Art gewonnene Ausdruck genau die Anzahl der Primzahlen zwischen $m$ und $2 m$, wenn $2 m<p_{s+1}^{2}$ vorausgesetzt wird, an. Es folgt dies unmittelbar aus der Gleichung $[2 x]-[x]=\{x\}$. 
Das sogenannte Umkehrungsproblem in etwas erweiterter Form ausgesprochen möge als $\mathrm{zw}$ eites $\mathrm{B}$ eispiel angeführt werden.

Es bedeute die Zahl $N$ irgend ein Multiplum der zueinander theilerfremd angenommenen Zahlen $n_{1}, n_{2}, \ldots, n_{s}$; ferner sei unter $d$ ein Theiler von $N$ verstanden, welcher die ganze positive Zahl $m$ nicht überschreitet and die Form $\prod_{i=1}^{i=s} n_{i}^{k_{i}}$ besitzt, wobei die Exponenten $k_{i}$ irgend welche, für jeden Theiler $d$ natürlich bestimmte ganze Zahlen, die Null miteingeschlossen, vorstellen. Bezeichnet endlich $f(x, y, z)$ eine willkürlich gegebene Function von drei Argumenten und $\varepsilon$ eine beliebige ganze Zahl, so darf die über alle eben definierten Theiler $d$ von $N$ erstreckte Summe

$$
\sum_{d} f\left(\left[\frac{m}{d}\right], \frac{N}{d}, \varepsilon d\right)=F^{\left(n_{1}, \ldots, n_{\mathrm{e}}\right)}(m, N, \varepsilon)
$$

gesetzt werden, weil sie eine völlig bestimmte Funetion der Zahlen $m, N, \varepsilon$ und $n_{1}, \ldots, n_{s}$ darstellt, welche übrigens der Kürze wegen, wenn betreffs der Zahlen $n_{1}, \ldots, n_{s}$ kein Zweifel herrschen kann, auch mit $F(m, N, \varepsilon)$ bezeichnet werden mag.

Es handelt sich darum, die Summe

$$
\sum_{\alpha} f\left(\left[\frac{m}{\alpha}\right], \frac{N}{\alpha}, \varepsilon \alpha\right)
$$

welche sich bloß auf alle die Werte $\alpha$ derjenigen Theiler $d$ bezieht, die durch keine der $s^{\prime}$ der Reihe $n_{1}, \ldots, n_{s}$ entlehnten Zahlen $v_{1}, \ldots, v_{s^{\prime}}$ theilbar sind, durch die Summen $F^{\prime}$ auszudrücken.

Auf Grund der Formel (I) in Nr. 1 ergibt sich, wenn man als gegebenes System $T$ die Summe $F(m, N, \varepsilon)$ und als Untersystem $r^{\text {ter }}$ Art $T_{i_{1} \ldots i_{r}}$ die Summen $F\left(\left[\frac{m}{\nu_{i_{1}} \ldots \nu_{i_{r}}}\right], \frac{N}{\nu_{i_{1}} \ldots \nu_{i_{r}}}, \varepsilon \nu_{i_{1}} \ldots \nu_{i_{r}}\right)$ wählt, die Gleichung

$$
\begin{gathered}
\sum_{\alpha} f\left(\left[\frac{m}{a}\right], \frac{N}{a}, \varepsilon \alpha\right)=F(m, N, \varepsilon)-\sum_{v} F\left(\left[\frac{m}{v}\right], \frac{N}{\nu}, \varepsilon v\right)+ \\
+\sum_{v v^{\prime}} F\left(\left[\frac{m}{v v^{\prime}}\right], \frac{N}{v v^{\prime}}, \varepsilon v v^{\prime}\right)-\cdots+\cdots,
\end{gathered}
$$

deren linke Seite natürlich nichts anderes, als die Function $F^{v^{\prime}{ }^{\prime}+y^{\prime}}, \cdots, v_{*}(m, N, \varepsilon)$ vorstellt, falls die nach Ausscheidung der Zahlen $v_{1}, \ldots, v_{s}$ übrigbleibenden Zahlen der Reihe $n_{1}, \ldots, n_{s}$ mit 
$v_{s^{\prime}+1}, \ldots, v_{s}$ bezeichnet werden. Man erhält hieraus speciell für $s^{\prime}=s$ die Gleichung

$$
\text { (III) } f(m, N, \varepsilon)=F(m, N, \varepsilon)-\sum_{n} F\left(\left[\frac{m}{n}\right], \frac{N}{n}, \varepsilon n\right)+\cdots,
$$

welche, falls $\operatorname{man} m=\infty, N=0$ und $\varepsilon=1$ setzt und für $n_{1}, n_{2}, n_{3}, \ldots$ alle Primzahlen nimmt, selbstrerständlich unter Voraussetzung der absoluten Convergenz der auftretenden Reihen in die von Herrn $\mathrm{Paul} \mathrm{Bachmann}{ }^{1}$ ) als "Umkehrungsprincip“ bezeichnete Relation übergeht.

Es ist klar, dass, wenn umgekehrt die Function $F^{\prime}$ willkürlich angenommen und die Gleichung (III) zur Definition der Function $f$ benützt wird, sich ohne weiteres aus der Beziehung (III) die frühere Ausgangsrelation (II) ableiten lässt, so dass die beiden Gleichungen (II) und (III) als äquivalent zu betrachten sind. Es ist jedoch hierbei wohl zu beachten, dass sich die Summen auf der rechten Seite einer jeden Definitionsgleichung für $f\left(\left[\frac{m}{d}\right], \frac{N}{d}, \varepsilon d\right)$ nur auf jene Zahlen der Reihe $n_{1}, \ldots, n_{s}$ und deren Combinationen zu erstrecken haben, welche Theiler von $\frac{N}{d}$ sind.

Um diese Behauptung nachzuweisen, hat man nur alle Definitionsgleichungen derart anzuschreiben, dass die denselben Combinationen von $n_{1}, \ldots, n_{s}$ entsprechenden Glieder untereinander zu stehen kommen, und hierauf die rechten Seiten colonnenweise zu addieren. Betrachtet man dann eine bestimmte Function $F\left(\left[\frac{m}{d}\right], \frac{N}{d}, \varepsilon d\right)$, in welcher der Divisor $d$ von $N$ blob durch die Zahlen $n_{i_{2}}, \ldots, n_{i_{r}}$ der Reihe $n_{1}, \ldots, n_{s}$ theilbar ist, so kommt diese Function genau in den Colonnen, die durch die Reihe $1, n_{i_{1}}, \ldots, n_{i_{r}}$ und durch die Combinationen dieser Zahlen charakterisiert sind, je einmal und nur einmal vor. Im Ganzen tritt sie also $1-\left(\begin{array}{l}r \\ 1\end{array}\right)+\left(\begin{array}{l}r \\ 2\end{array}\right)-\cdots$ mal, d. h. überhaupt nicht auf.

Dieser Zusammenhang zwischen den Gleichungen (II) und (III) gestattet die folgende Anwendung:

Wählt man für jedes Wertepaar $N$ und $\varepsilon$ die Function $F(m, N, \varepsilon)$ gleich $m$, so nimmt die rechte Seite der Relation (III) die Gestalt $m-\sum_{n}\left[\frac{m}{n}\right]+\cdots$ an, und es stellt somit die linke Seite, d. h. die Function $f(m, N, \varepsilon)$ gemäß dem durch den Ausdruck (I) dieser Nummer vermittelten Satze die Anzahl der Zahlen,

1) Zahlentheorie, II. Theil, 1894, p. 311. 
die nicht größer als $m$ und durch die Divisoren $n_{1}, \ldots, n_{s}$ von $N$ nicht theilbar sind, dar. Unter diesen Voraussetzungen vereinigt dann der durch die Gleichung (II) gegebene Satz zwei in der bereits oben erwähnten Notiz: Zur Verallg. d. Fct. $\varphi(m)$ etc. $\left.{ }^{1}\right)$ als wesentlich von einander verschieden aufgefasste Relationen. Um die erste zu erhalten, hat man nur $N=\prod_{i=1}^{i=s} n_{i}$ zu setzen; die zweite ergibt sich durch die Specialisierung $N=\prod_{i=1}^{i=s} n_{i}^{\lambda_{i}}$ unter der Annahme $n_{i}^{\lambda_{i}}>m(i=1,2, \ldots, s)$. Nimmt man endlich $m=N$ und für $n_{1}, \ldots, n_{s}$ die verschiedenen Primfactoren von $m$, so drückt die Gleichung (II) den bekannten Satz $\sum_{d} \varphi(d)=m$ aus.

Es möge endlich noch eine dritte Anwendung der Methode des Ausscheidens und Hinzufügens hervorgehoben werden.

Die Gesammtheit derjenigen Congruenzen aller Grade, welche in Bezug auf den Primzahlmodul $p$ die Wurzel $\alpha_{i}$ besitzen und bei denen der Coefficient der höchsten Potenz von $x$ gleich der Einheit angenommen worden ist, bilden bezüglich der Multiplication als Operation eine Gruppe $T_{i}$, deren Elemente sämmtlich in der Form $\left(x-\alpha_{i}\right) f(x) \equiv 0$ enthalten sind.

Um den Complex jener Congruenzen, welche keine der verschiedenen Wurzeln $\alpha_{1}, \ldots, \alpha_{s}$ besitzen, zu erhalten, hat man nur aus der Gesammtheit $T$ aller Congruenzen aller Grade die Gruppen $T_{i}(i=1,2, \ldots, s)$ auszuscheiden. Das gesuchte Resultat stellt sich somit in der Gestalt (I) der Nr. 1 dar, wenn man einem bekannten Satze entsprechend für $T_{i_{1}, \ldots, i_{\sigma}}$ den Inbegriff der in der Form $\left(x-a_{i_{1}}\right) \ldots\left(x-a_{i_{r}}\right) f(x) \equiv 0$ enthaltenen Congruenzen setzt.

Behält man bloß die Congruenzen $n^{\text {ten }}$ Grades bei und bezeichnet man mit $f_{k}^{(i)}(x)\left(i=1,2, \ldots, p^{k}\right)$ alle modulo $p$ verschiedenen Polynome $k^{\text {ten }}$ Grades, in denen der Coefficient ron $x^{k}$ gleich der Einheit ist, mit $\Phi(f(x))$ aber eine modulo $p$ invariante Function, so sieht man ohne weiteres die Richtigkeit der Äquivalenz

$$
\begin{gathered}
\sum_{a} \Phi\left(f_{n}^{(\alpha)}(x)\right) \sim \sum_{j=1}^{p^{n}} \Phi\left(f_{n}^{(j)}(x)\right)-\sum_{i} \sum_{j=1}^{p^{n-1}} \Phi\left(\overline{x-\alpha_{i}} f_{n-1}^{(j)}(x)\right)+ \\
+\sum_{i i^{\prime}} \sum_{j=1}^{p^{n-2}} \Phi\left(\overline{x-\alpha_{i}} \overline{x-\alpha_{i}^{\prime}} f_{n-2}^{(j)}(x)\right)-\cdots+\cdots
\end{gathered}
$$

1) 1. c. p. 345 Z. 5 v. u. und p. 346 Z. 8 v. o. 
ein, wo das $\alpha$ alle diejenigen Werte der Reihe $1,2, \ldots, p^{n}$ anzunehmen hat, für welche die zugehörigen Congruenzen $f_{n}^{(\alpha)}(x) \equiv 0$ $(\bmod p)$ keine der $W$ urzeln $\alpha_{1}, \ldots, a_{s}$ besitzen. $\left.{ }^{1}\right)$

Die Äquivalenz (IV) ist grundlegend für die Theorie der wurzellosen Congruenzen und ich werde demnächst in einem Autsatze diesen Gegenstand eingehender erörtern.

\section{Abschnitt. Über die Theorie Abel'scher Gruppen.}

Nr. 1. Über Gruppen im Allgemeinen.

In der vorliegenden Nr. sollen einige bekannte Sätze und Definitionen aus der Gruppentheorie zusammengestellt werden, welche im weiteren Verlaufe der Untersuchungen eine Rolle zu spielen. geeignet sind.

Ein System $T$ von wohldefinierten untereinander verschiedenen Elementen $A_{1}, A_{2}, A_{3}, \ldots$, deren Anzahl auch unbegrenzt sein kann, soll die folgenden drei Eigenschaften besitzen:

1. Aus je zwei Elementen von $T$ lässt sich mit Hilfe einer gegebenen Vorschrift $\mathfrak{B}$ ein neues Element desselben Systemes eindeutig herleiten. Das Resultat der Verknuipfung der beiden Elemente $A_{i}$ und $A_{k}$ entsprechend der Vorschrift $\mathfrak{B}$ möge mit

bezeichnet werden.

$$
\mathfrak{B}\left(A_{i}, A_{k}\right)
$$

2. Aus der Identität

$$
\mathfrak{B}\left(A_{i}, A_{k}\right)=\mathfrak{B}\left(A_{i}, A_{h}\right) \text { oder auch } \mathfrak{B}\left(A_{k}, A_{i}\right)=\mathfrak{B}\left(A_{h}, A_{i}\right)
$$

folgt die Übereinstimmung der Elemente $A_{l}$ und $A_{h}$.

3. Es gilt das associative Gesetz:

$$
\mathfrak{B}\left(\mathfrak{B}\left(A_{i}, A_{k}\right), A_{h}\right)=\mathfrak{B}\left(A_{i}, \mathfrak{B}\left(A_{k}, A_{h}\right)\right) .
$$

Ein solches System $T$ wird, je nachdem es eine unendliche oder endliche Anzahl von Elementen umfasst, eine unendliche oder endliche Gruppe beziuglich der Vorschrift $\mathfrak{B}$ genannt.

So bildet z. B. die Gesammtheit der Substitutionen von $n$ Elementen eine endliche Gruppe, wenn man die Zusammensetzung je zweier Substitutionen zu einer einzigen als Vorschrift $\mathfrak{B}$ betrachtet, während der Inbegriff aller zu einer gegebenen Zahl $m$ theilerfremden ganzen Zahlen eine unendliche Gruppe vorstellt, bei welcher die Multiplication als Vorschrift $\mathfrak{B}$ dient.

1) Vergl. den Jahresbericht der Math. Vereinigung 1894, p. 109 und Wiener Sitzungsberichte CIII: Über die Anzahl derjenigen ganzen ganzzahligen Functionen $n^{\text {ten }}$ Grades von $x$, welche in Bezug auf einen gegebenen Primzablmodul eine vorgeschriebene Anzahl von Wurzeln besitzen.

Monatih. f. Mathematik u. Physiks. VII. Jahrg. 
Man kann die eben für eine Gruppe gegebene Definition dadurch erweitern, dass man in den Bedingungen 1., 2. und 3 . an die Stelle der absoluten Gleichheit oder Identität zweier Elemente die relative Gleichbeit derselben, d. h. ibre Äquivalenz in Bezug auf eine Eigenschaft $\mathfrak{x}$ treten lässt.

Gelingt es nämlich alle Elemente eines Systemes $T$ der Art in Klasen $T_{i}(i=1,2,3, \ldots)$ einzutheilen, dass je 2 Individuen von $T$ in eine und dieselbe Klasse oder in zwei verschiedene Klassen eingereiht werden, jenachdem sie eine bestimmte Eigenschaft $\mathfrak{x}$ besitzen oder nicht, so können alle Elemente einer und derselben Klasse einander hinsichtlich $\mathfrak{x}$ vertreten, sind also in Bezug auf $x$ äquivalent. Indem man aus jeder Klasse $T_{i}$ ein Element $A_{i}$ beliebig herausgreift, gelangt man zu dem Begriffe eines innerhalb des Complexes $T$ vollständigen Systemes $\Gamma$ von untereinander in Bezug auf $\mathfrak{x}$ nicht äquivalenten Elementen $A_{1}, A_{2}, A_{3}, \ldots$.

Genügt auherdem das System $T$ den Bedingungen 1., 2. und 3., in denen natürlich für jede Gleichung eine Äquivalenz in Bezug. auf $\mathfrak{x}$ zu setzen ist, so soll gesagt werden, dass das vollständige System $\Gamma$ nicht äquivalenter Elemente $A_{1}, A_{2}, \ldots$ eine Gruppe hinsichtlich der Vorschrift $\mathfrak{B}$.bilde.

Die Berechtigung hierzu ist unmittelbar einzusehen; denn existiert ein Verfahren $\mathfrak{V}^{\prime}$ um aus irgend einem Elemente von $T$ das ihm äquivalente des Systemes $\Gamma$ herzuleiten, so bildet der Complex $\Gamma$ auch im früheren. Sinne eine Gruppe bezüglich der Vorschrift $\mathfrak{B}$ and $\mathfrak{B}^{\prime}$ zusammengenommen.

In diesem Sinne constituiert z. B. das in Bezug auf den Primzahlmodul $p$ genommene vollständige Restsystem ohne die Null

$$
1,2,3, \ldots, \overline{p-1}
$$

eine Gruppe hinsichtlich der Multiplication.

Einerseits lässt sich nämlich das System $T$ aller durch $p$ nicht theilbaren Zahlen auf Grund der Eigenschaft $x$ der ganzen Zahlen bei der Division durch $p$ einen bestimmten Rest zu lassen, in Congruenz-Klassen eintheilen; andererseits genügt es den Bedingungen 1., 2. und 3., wenn man für jede Gleichung eine Congruenz modulo $p$ setzt und für die Vorschrift $\mathfrak{B}$ die Multiplication nimmt.

Der einfacheren Ausdrucksweise halber soll im Folgenden die Vorschrift $\mathfrak{B}$ Multiplication genannt und das Resultat $\mathfrak{B}\left(A_{i}, A_{k}\right)$ der Verknüpfung der beiden Elemente $A_{i}$ and $A_{k}$ kurz mit $A_{i} A_{i}$ bezeichnet werden, wie man dies bekanntlich bei den Substitutionen zu thun pflegt. Endlich möge das Gleichheitszeichen in dem angegebenen, erweiterten Sinne eines Äquivalenzzeichens Verwendung tinden. 
Von selbst leuchtet nunmehr ein, was unter dem Producte von 2 oder mehreren Elementen (Factoren) und unter einer Potenz $A_{i}^{3 u}$ eines Elementes $A_{i}$ zu verstehen sein wird.

Es werden im Folgenden nur endliche Gruppen betrachtet. Die Anzahl der Elemente einer solchen Gruppe $\Gamma$ wird ihr Grad genannt.

Auf Grund des Postulates 2. erkennt man ohne Weiteres, dass das Product $A_{i} X$ mit dem Factor $X$ zugleich die endliche Gruppe $\Gamma$ durchlauft. Es existiert also insbesondere innerhalb der Gruppe $\Gamma$ ein und nur ein Element $E$, welches die Gleichung:

$$
A_{i} E=A_{i}
$$

befriedigt. Nach Multiplication beider Seiten derselben mit $A_{k}$ folgt vermöge der Bedingungen 3. und 2. die Beziehung

$$
E A_{k}=A_{k} \text {, }
$$

aus welcher sich wiederum die Gleichheit der Elemente $A_{k} E A_{k}$ und $A_{k} A_{k}$, also 'auch diejenige von $A_{k} E$ und $A_{k}$ ergibt.

Jedes Individuum bleibt mithin nach der Multiplication mit $E$ ungeändert. Das Element $E$ wird daher passend die.Einheit der Gruppe $\Gamma$ genannt and mit 1 bezeichnet werden.

Da die Anzahl der Elemente der Gruppe $\Gamma$ beschränkt, die Anzahl der Potenzen'eines jeden Elementes $A_{i}$ aber unbeschränkt ist, müssen schließlich bei fortgesetztem Potenzieren zwei Potenzen von $A_{i}$, z. B. $A_{i}^{\mu+\nu}$ und $A_{i}^{v}$ einander gleich werden, woraus die Beziehung $A_{i}^{n}=1$ folgt.

Der niedrigste positive Exponent $\delta$, der für $\mu$ gesetzt, diese Gleichung betriedigt, wird der Grad des Elementes $A_{i}$ genannt und es ist klar, dass die Gleichung $A_{i}^{u}=1$ dann und nur dann bestehen kann, wenn $\mu$ ein Multiplum von $\delta$ ist.

Es ergibt sich nunmehr von selbst, was man unter einer Potenz mit negativem Exponenten zu verstehen haben wird.

Alle Elemente der Reihe

$$
1, A_{i}, A_{i}^{2}, \ldots, A_{i}^{\delta-1}
$$

sind voneinander verschieden und es leuchtet ein, dass dieses Elementensystem selbst eine Gruppe bezüglich der Multiplication bildet. Ihrer ausgezeichneten Form halber soll diese Gruppe eine reguläre heissen.

Jede Gruppe $P$ in Bezug auf dieselbe Vorschritt $\mathfrak{B}$, deren Elemente sämmtlich unter denen von $\Gamma$ enthalten sind, wird eine Untergruppe von $\Gamma$ genannt; es ist klar, dass hierzu das Bestehen 
der Bedingung 1. für alle Elemente von $P$ ausreicht, da ja die Postulate 2, und 3. von selbst befriedigt erscheinen.

Bezeichnet man mit dem Symbol $P A_{j}$ den Complex der Elemente ron $P$ nach Multiplication eines jeden mit $A_{j}$, so kann man, wie bekanntlich eine einfache Überlegung lehrt, die Gruppe $\Gamma^{\top}$ durch die Systeme

$$
P ; P A_{1} ; P A_{2} ; \ldots ; P A_{k}
$$

darstellen, falls nur das Element $A_{i}(i=1,2, \ldots, k)$ sich in keinem der Systeme $P A_{j}\left(j=0,1,2, \ldots,(i-1) ; A_{0}=1\right.$ vorfindet.

Daraus folgt, dass der Grad einer jeden Untergruppe, also. auch speciell der Grad eines jeden Elementes von $T$ ein Theiler des Grades von $\Gamma$ sein muss.

So oft die Gruppe $F$ sich in der Form (2) darstellen lässt, soll gesagt werden, dass die Gruppe $\Gamma$ als durch Composition des Systemes $P$ mit dem Systeme $1, A_{1}, \ldots, A_{k}$ entstanden gedacht werden kann, wobei jedoch wohl zu beachten ist, dass sich bei der

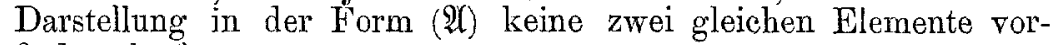
finden dürfen.

Tritt zu den drei oben angegebenen Eigenschaften einer endlichen Gruppe $\Gamma$ das Bestehen des commutativen Gesetzes

$$
A_{i} A_{k}=A_{k} A_{i}
$$

zwischen je zwei Elementen als vierte hinzu, so heißt die Gruppe eine Abel'sche. ${ }^{1}$ )

Es ist klar, dass jede Untergruppe einer Abel'schen Gruppe wieder eine solche vorstellt.

\section{Nr. 2. Über das Basissystem einer Abel'schen Gruppe, deren Grad eine Primzahlpotenz ist.}

Es soll zunächst, und zwar auf inductivem Wege, gezeigt werden, dass jede Abel'sche Gruppe H, deren Grad eine Primzahlpotenz $p^{\pi}$ ist, sich durch Composition aus lauter regulären Gruppen erzeugen lässt.

Nach einer Bemerkung der vorigen Nummer kann die Gruppe II nur Elemente umfassen, deren Gradzahlen als Theiler von $p^{\pi}$ selbst Potenzen von $p$ sind. Sei $p^{\alpha_{1}}$ der höchste Grad, welcher von den Elementen der Gruppe $H$ überhaupt erreicht wird, und eines derjenigen Elemente, welche diesen Grad tbatsäichlich besitzen, möge mit $A_{1}$ bezeichnet werden.

1) H. Weber, ellipt. Fct. u. algebr. Zahlen. 1891, p. 177; oder auch Lehrbuch der Algebra. $189 \tilde{0}$, p. 476. 
Angenscheinlich hat dann das System

$\left(\mathrm{E}_{1}\right)$

$$
A_{1}^{k_{1}} \quad\left(k_{1}=0,1, \ldots,\left(p^{\alpha_{1}}-1\right)\right)
$$

die beiden folgenden Eigenschaften:

1. Es stellt eine Gruppe dar.

2. Die Zahl $p^{\alpha_{1}}$ ist der niedrigste positive Exponent, auf den alle Elemente von $H$ erhoben werden müssen, um ausnahmslos gleich der Einheit $A_{0}$ zu werden.

Ist die Gruppe $\mathrm{H}$ identisch mit $\left(\mathrm{E}_{1}\right)$, so versteht sich die zu beweisende Behauptung von selbst; denn in diesem Falle kann die Gruppe $H$ als durch Composition des Systemes $\left(\mathrm{F}_{1}\right)$ mit der Einheit $A_{0}$ erzengt gedacht werden.

Finden sich hingegen innerhalb des Complexes $\mathrm{H}$ neben den Elementen von $\left(\mathrm{E}_{1}\right)$ noch andere vor, so lässt sich eine Zahl $p^{\alpha_{2}}$, nicht größer als $p^{a_{1}}$, und entsprechend derselben ein Element $A_{2}$ vom Grade $p^{\alpha_{2}}$ der Gruppe $H$ der Art bestimmen, dass das System

$$
A_{1}^{k_{1}} A_{2}^{k_{2}} \quad\left(\begin{array}{rl}
k_{i} & =0,1, \ldots,\left(p^{a_{i}}-1\right) \\
i & =1,2
\end{array}\right)
$$

wieder die beiden Bedingungen erfüllt:

1. Es stellt eine Gruppe dar.

2. Die Zahl $p^{\alpha_{2}}$ ist der niedrigste positive Exponent, auf den alle Elemente von $\mathrm{H}$ erhoben werden müssen, um ausnahmslos die Form $A_{1}^{k_{1}}$ anzunehmen.

Der Nachweis hierfür soll gleich ganz allgemein geführt werden:

Angenommen, es sei, wie dies bei dem Systeme $\left(E_{1}\right)$ der Fall ist, gelungen, aus der Gruppe $\mathrm{H}(p-1)$ Elemente $A_{i}$ mit nicht zunehmenden Gradzahlen $p^{\alpha_{i}}(i=1,2, \ldots, \overline{p-1})$ der Art auszuwählen, dass das System

$$
\left(\mathrm{E}_{\varrho-1}\right) \quad A_{1}^{k_{1}} A_{2}^{k_{2}} \ldots A_{\varrho-1}^{k_{\varrho-1}} \quad\left(\begin{array}{c}
k_{i}=0,1, \ldots,\left(p^{\alpha_{i}}-1\right) \\
i=1,2, \ldots, \overline{\rho-1}
\end{array}\right)
$$

die beiden folgenden Bedingungen erfüllt:

1. Es ist selbst eine Gruppe; enthält also keine zwei gleichen Elemente, oder keine Potenz von $A_{i}$ hat die Form

$$
A_{0} A_{1}^{k_{1}} \ldots A_{i-1}^{k_{i-1}} \quad(i=1,2, \ldots, \overline{\rho-1}),
$$

ausgenommen natürlich die $0^{\text {te }}$, die $\pm\left(p^{\alpha_{i}}\right)^{\text {te }}, \ldots$ Potenz;

2. Die Zahl $p^{\alpha_{i}}$ ist der niedrigste positive Exponent, auf den alle Elemente von $\mathrm{H}$ erhoben werden müssen, um ausnahmslos die Form $A_{0} A_{1}^{k_{1}} \ldots A_{i-1}^{k_{i-1}} \quad(i=1,2, \ldots, \rho-1)$ zu erhalten; 
so lässt sich, falls die Gruppe $H$ durch das System $\left(E_{e-1}\right)$ noch nicht erschöpft ist, innerhalb derselben ein $\rho^{\text {tes }}$ Element $A_{\varrho}$ vom Grade $p^{\alpha} \varrho$, der nicht größer als $p^{\sigma^{\circ} \varrho-1}$ ist, der Art finden, dass das System

$$
A_{1}^{k_{1}} \ldots A_{\varrho}^{k_{\varrho}} \quad\left(\begin{array}{c}
\left.k_{i}=0,1, \ldots, p^{a_{i}}-1\right) \\
i=1,2, \ldots, \rho
\end{array}\right)
$$

wieder die Forderungen 1. und 2., nachdem in denselben die Zahl $\overline{\rho-1}$ durch $\rho$ ersetzt worden ist, erfüllt, dass es also auf Grund der Bedingung 1. als durch Composition der $\rho$ regulären Gruppen

$$
1, A_{i}, \ldots, A_{i}^{p^{\alpha_{i}}-1}\left(\begin{array}{c}
i=1,2, \ldots, \rho \\
\rho>1
\end{array}\right)
$$

entstanden gedacht werden kann.

In der That, es muss nach den gemachten Annahmen einen niedrigsten positiven Exponenten $\mu$, nicht größer als $p^{\alpha_{\varrho}-1}$, geben, auf welchen alle Elemente der Gruppe $H$ erhoben werden miissen, um ausnahmslos gleich Elementen von $\left(\mathbf{E}_{o-1}\right)$ zu werden; denn gemäß der Voraussetzung 2. findet dies sicher bereits beim Exponenten $p^{\alpha} \varrho-1$ statt.

Diese Zahl $\mu$ muss eine Potenz $p^{\alpha_{\varrho}}$ von $p$ sein; denn bestünde die Gleichung

$$
\mu=\mu^{\prime} p^{\alpha_{e}} \quad\left(\begin{array}{l}
\mu^{\prime}>1 \\
\mu^{\prime} \text { nicht } \equiv 0(\bmod p)
\end{array}\right),
$$

so liefe sich eine ganze Zahl $\eta$ gemäß der Congruenz $\eta \mu^{\prime} \equiv 1$ $\left(\bmod p^{\sigma}\right)$ bestimmen, und der Exponent $\eta \mu^{\prime} p^{\alpha}$ e, d. h. auch schon die Zahl $p^{\alpha} \varrho$ hätte gegen die Annahmè dieselbe Eigenschaft, wie $\mu$.

Aus der Bedeutung von $p^{\alpha} e$ ist klar, dass im Systeme $\mathrm{H}-\mathrm{E}_{\varrho-1}$ wenigstens ein Element $C$ vorkommt, welches erst auf $\left(p^{\alpha} \varrho\right)^{\text {te }}$ Potenz erhoben und nicht früher die Form

$$
A_{1}^{k_{1}} A_{2}^{k_{2}} \ldots A_{\varrho-1}^{k_{\varrho}-1}
$$

annimmt. Aus der Gleichung

$$
C^{p^{\alpha_{\varrho-1}}}=A_{1}^{k_{1} p^{\alpha_{Q-1}-\alpha_{\varrho}}} \ldots A_{\varrho-2}^{k_{\varrho-2} p^{\alpha_{Q-1}-\alpha_{\varrho}}} A_{\varrho Q-1}^{k_{Q-1} p^{\alpha_{Q-1}-\alpha_{\varrho}}}
$$

folgt aber, dass das Element $A_{\varrho-1}^{k_{Q-1} p^{\alpha_{Q-1}-\alpha_{Q}}}$, weil die linke Seite derselben nach der Bedingung 2. die Form $A_{1}^{k_{1}^{\prime}} \ldots A_{o-2}^{k_{e-2}^{\prime}}$ besitzt, ebenfalls dieselbe Form hat, dass also gemäß der Forderung 1. die 
Zahl $k_{\rho-1}$ ein Multiplum von $p^{\alpha} \varrho$ ist. Unter Berücksichtigung dieses Umstandes erbält man die Gleichung

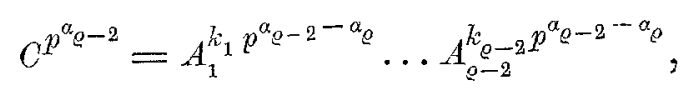

aus welcher sich analog, wie früher, die Theilbarkeit des Exponenten $k_{e-2}$ durch $p^{a_{o}}$ ergibt. In dieser Weise fortfahrend erkennt man, dass allgemein die Zahl $k_{i}$ gleich $\mu_{i} p^{\alpha} e^{e}$ $(i=1,2, \ldots, \rho-1)$ ist, wobei mit $\mu_{i}$ eine ganze $Z$ ahl bezeichnet wird.

Das Element

$$
C A_{1}^{-\mu_{1}} \ldots A_{Q-1}^{-\mu_{Q-1}}
$$

besitzt mithin den Grad $p^{\alpha_{0}}$ und nimmt auf keine niedrigere Potenz, als die $\left(p^{a_{Q}}\right)^{\text {te }}$ erhoben, die Gestalt $A_{1}^{k_{1}} \ldots A_{e_{-1}}^{k_{Q-1}}$ an; es kann somit als gesuchtes Element $A_{o}$ betrachtet werden.

Von dem auf diese Art gefundenen Systeme $\left(\mathrm{E}_{\varrho}\right)$ kann man in analoger Weise zu einem Systeme $\left(\mathrm{E}_{\ell+1}\right)$ gelangen u. s. f., bis die ganze Gruppe $\mathrm{H}$ erschöpft erscheint.

Man ist also zu dem folgenden Theorem gelangt:

Es gibt innerhalb einer jeden Abel'sehen Gruppe H, deren Grad eine Primzahlpotenz $p^{x}$ ist, Elemente $A_{i}$ vom Grade $p^{\alpha_{i}}(i=1,2, \ldots, s)$ der Art, dass jedes Element der Gruppe H in der (1) Form

$$
A_{1}^{k_{1}} A_{2}^{k_{2}} \ldots A_{s}^{k_{s}} \quad\left(\begin{array}{c}
k_{i}=0,1, \ldots,\left(p^{a_{i}}-1\right) \\
i=1,2, \ldots, s
\end{array}\right)
$$

und zwar jedes nur einmal enthalten ist.

Der Inbegriff solcher Elemente $A_{1}, \ldots, A_{s}$, die alle natürlich von der Einheit versehieden sind, soll ein Basissystem der A bel'schen Gruppe H genannt werden.

Schon aus dem oben geführten Existenzbeweis für dasselbe geht hervor, dass es verschiedene Basissysteme einer und derselben A bel'schen Gruppe gibt und es ist von Interesse, ihre Anzahl zu bestimmen.

Augenscheinlich ist die Anzahl $Z_{\sigma}$ derjenigen Elemente innerhalb der Gruppe $\mathrm{H}$, welche den vorgegebenen Grad $p^{\sigma}$ besitzen, völlig unabhängig von der Wahl des Basissystemes. Überdies lässt sich die Zahl $Z_{\sigma}$ leicht auf Grund des Satzes (1) angeben.

Denkt man sich nämlich die Elemente des Basissystemes $A_{1}, \ldots, A_{s}$ wieder der Art geordnet, dass ihre Gradzablen nicht 
zunehmen, so existiert, wenn der Exponent $\sigma$ gröber als $\alpha_{1}$ ist, überhaupt kein Element vom Grade $p^{\sigma}$.

Andernfalls mögen $i$ der Exponenten $\alpha_{j}(j=1,2, \ldots, s)$ nicht kleiner als $\sigma$ sein. Alsdann finden sich innerhalb jeder der Gruppen

$$
1, A_{j}, \ldots, A_{j}^{p^{\alpha_{j}}-1} \quad(j=1,2, \ldots, i)
$$

genau $p^{\sigma}$ Elemente, welche einen nicht größeren Grad als $p^{\sigma}$ besitzen, weil ein jedes derartige Element die Form $A_{j}^{k p^{\alpha_{j}}-\sigma}$ $\left(k=0,1, \ldots,\left(p^{\sigma}-1\right)\right)$ haben muss. Die Untergruppe

$$
A_{1}^{k_{1}} \ldots A_{i}^{k_{i}} \quad\left(\begin{array}{l}
k_{j}=0,1, \ldots,\left(p^{\alpha_{j}}-1\right) \\
j=1,2, \ldots, i
\end{array}\right)
$$

entliält daher $p^{i \sigma}$, die ganze Gruppe $\mathrm{H}$ aber $p^{i \sigma+\alpha_{i+1}+\cdots+\alpha_{s}}$ derartig'e Elemente.

Die Anzahl der Elemente von einem nicht großeren Grade als $p^{\sigma-1}$ ist nach dem eben gewonnenen Resultate gleich $p^{i(\sigma-1)+a_{i+1}+\cdots+\alpha_{s}}$, weil der Exponent $(\sigma-1)$ nicht kleiner als $\alpha_{i+1}$ sein kann.

Bildet man die Differenz der beiden gefundenen Zahlen, so erhält man die gesuchte Zahl $Z_{\sigma}$ und man ist somit zu dem folgenden Satz gelangt:

Bezeichnet man mit $i$ die Anzahl der Zahlen $\alpha_{j}(j=1,2, \ldots, s)$, welchenichtkleiner als $\sigma$ sind, so ist die Anzahl der Elemente rom Grade $p^{\sigma}$ innerhalb der Gruppe H gleich dem Producte

$$
p^{i \sigma+a_{i+1}+\cdots+a_{s}} \quad\left(1-\frac{1}{p^{i}}\right) .
$$

Sei die Gruppe $H$ mit Hilte eines zweiten Basissystemes $A_{1}^{\prime}, \ldots, A_{s^{\prime}}^{\prime}$, dessen Elemente der Reibe nach die nicht zunehmenden Gradzahlen $p^{\alpha_{1}^{\prime}}, p^{\alpha_{2}^{\prime}}, \ldots, p^{\alpha_{\partial^{\prime}}^{\prime}}$ besitzen mögen, dargestellt.

Wählt man die positive Zahl $\sigma$ weder größer als $\alpha_{s}$, noch größer als $\alpha_{s^{\prime}}^{\prime}$, so gibt das Theorem (2) bei Zugrundelegung des Basisssystemes $A_{j}$, bezw. $A_{j^{\prime}}^{\prime}$, für $Z_{\sigma}$ den Ausdruck $p^{s(\sigma-1)}\left(p^{s}-1\right)$, bezw. $p^{s^{\prime}(\sigma-1)}\left(p^{s^{\prime}}-1\right)$, woraus unmittelbar die Gleichheit der beiden Zahlen $s$ und $s^{\prime}$ folgt.

Seien ferner genau $j_{s}$ von den Exponenten $\alpha_{j}(j=1,2, \ldots, s)$ gleich $\alpha_{s}$ und $j_{s}^{\prime}$ der Exponenten $\alpha_{j}^{\prime}(j=1, \ldots, s)$ gleich $\alpha_{s}^{\prime}$. 
Angenommen nun, es wäre $\alpha_{s}<\alpha_{s}^{\prime}$, so erhielte man, die Zahl $\sigma$ gleich $\alpha_{s}+1$ gesetzt, wieder nach dem Satze (2) die Gleichung

$$
p^{\left(s-j_{s}\right)(\sigma-1)+j_{s} \alpha_{s}}\left(p^{s-j_{s}}-1\right)=p^{s(\sigma-1)}\left(p^{s}-1\right),
$$

aus welcher der Widerspruch $j_{s}=0$ sich ergeben würde. Es kann mithin die Zahl $\alpha_{s}$ nicht kleiner als $\alpha_{s}^{\prime}$ und aus demselben Grunde die Zahl $\alpha_{s}^{\prime}$ nicht kleiner als $\alpha_{s}$ sein, d. h. es muss die Gleichung $\alpha_{s}=\alpha_{s}^{\prime}$ bestehen.

Indem man nun abermals $\sigma=\alpha_{s}+1$ setzt, erhält man nach dem eben angewandten Schlusse die Relation:

$$
p^{\left(s-j_{s}\right)(\sigma-1)+j_{s} \alpha_{s}}\left(p^{s-j_{s}}-1\right)=p^{\left(s-j_{s}^{\prime}\right)(\sigma-1)+j_{s}^{\prime} \alpha_{s}}\left(p^{s-j_{s}^{\prime}}-1\right),
$$

aus welcher wieder die Beziehung $j_{s}=j_{s}^{\prime}$ folgt.

In dieser Weise fortfahrend erkennt man die Identität der beiden Reihen $\alpha_{1}, \ldots, \alpha_{s}$ und $\alpha_{1}^{\prime}, \ldots, \alpha_{s^{\prime}}^{\prime}$; das heibt:

Die Gradzahlen der Individuen eines Basissystemes und die Anzahlder Basiselemente eines (3) jeden Grades innerhalb einer Abel'schen Gruppe, deren Grad eine Primzahlpotenz ist, sind unabhängig von der Wahl des Basissyistemes.

Es ist nunmehr leicht, die Anzahl der verschiedenen Basissysteme der betrachteten Abel'schen Gruppe H zu bestimmen.

In einem jeden in die bekannte Anordnung gebrachten Basissysteme $A_{1}^{\prime}, \ldots, A_{s}^{\prime}$ besitzt erstens das $p^{\text {te }}$ Element $A_{\varrho}^{\prime}$ nach dem Satze (3) nothwendiger Weise den Grad $p^{\alpha} \alpha_{Q}$, zweitens darf sich erst die $\left(p^{\alpha}\right)^{\text {te }}$ und keine frühere Potenz von $A_{\varrho}^{\prime}$ in dem Systeme

$$
A_{1}^{\prime k_{1}} \ldots A_{\varrho-1}^{\prime k_{e-1}} \quad\left(\begin{array}{c}
k_{i}=0,1, \ldots,\left(p^{\alpha_{i}}-1\right) \\
i=1,2, \ldots, \rho-1
\end{array}\right)
$$

vorfinden.

Umgekehrt lehrt das vorhin beim Existenzbeweise angewandte Verfahren, dass jedes Element $\bar{A}_{e}^{\prime}$, welches diese beiden Bedingungen erfüllt, wirklich als $p^{\text {tes }}$ Basiselement genommen werden kann, wie immer die vorangehenden Basiselemente $A_{1}^{\prime}, \ldots, A_{e_{-1}^{\prime}}^{\prime}$ gewählt worden sein mögen.

Um nun die Anzahl $y_{o}$ dieser für $\bar{A}_{o}^{\prime}$ möglichen Elemente zu

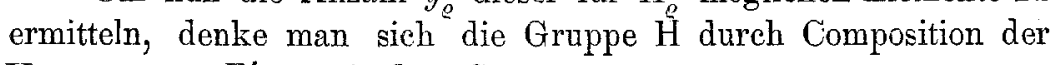
Untergruppe $\left(E_{e-1}^{\prime}\right)$ mit dem Systeme

$$
\left(\overline{\mathrm{E}}_{s-\varrho+1}^{\prime}\right) A_{\varrho}^{\prime k_{e}} A_{\varrho+1}^{\prime k_{\varrho+1}} \ldots A_{s}^{\prime k_{s}} \quad\left(\begin{array}{c}
k_{j}=0,1, \ldots,\left(p^{\alpha_{j}}-1\right) \\
j=\rho, \rho+1, \ldots, s
\end{array}\right)
$$


dargestellt. Jedes Element der Untergruppe $\left(\overline{\mathrm{E}}_{s-\varrho+1}^{\prime}\right)$ vom Grade $p^{\alpha}{ }^{\alpha}$ gibt mit jedem Elemente $\left(\mathrm{E}_{g-1}^{\prime}\right)$, dessen Grad nicht größer als $p^{\alpha} \varrho$ ist, combiniert, ein Element der verlangten Eigenschaft; und zwar sind dies die einzigen Elemente dieser Art, wie eine einfache Überlegung lehrt.

Man erhält also nach dem Satze (2) die Gleichung

$$
y_{\varrho}=p^{\sum_{j=\varrho}^{s} \alpha_{j}}\left(1-\frac{1}{p^{v_{\varrho}}}\right) \cdot p^{p^{\varrho-1} a_{\varrho}},
$$

wenn genau $\nu_{\varrho}-1$ von den auf $\alpha_{\varrho}$ folgenden Exponenten $\alpha_{\varrho+1}, \alpha_{\varrho+2}, \ldots, \alpha_{s}$ gleich $\alpha_{o}$ sind.

Die Anzahl $y_{o}$ bängt selbstverständlich nicht von der Natur der dem Elemente $A_{\rho}^{\prime}$ vorangehenden Basiselemente ab und es ergibt sich somit für die Gesammtzahl $y$ der nicht bloß durch die Anordnung der Elemente verschiedenen Basissysteme das Product

$$
\frac{\prod_{\varrho=1}^{\varrho=s} y_{\varrho}}{n_{1} ! n_{2} ! \ldots n_{r} !}
$$

wenn genau $n_{1}$ der Elemente $\alpha_{j}(j=1,2, \ldots, s)$ gleich $\overline{\alpha_{1}}$ oder $\alpha_{1}$; $n_{2}$ der Elemente $\alpha_{j}$ gleich $\bar{\alpha}_{2}$ oder $\alpha_{n_{1}+1}$ u. s. f., $n_{r}$ der Elemente $\alpha_{j}$ gleich $\bar{\alpha}_{r}$ oder $\alpha_{s}$ sind, wobei natürlich $\bar{\alpha}_{1}>\bar{\alpha}_{2} \cdots>\bar{\alpha}_{r}$ rorauszusetzen ist.

Berücksichtigt man die Relationen $v_{1+j_{1}}=n_{1}-j_{1}\left(j_{1}=0,1\right.$, $\left.\ldots, \overline{n_{1}-1}\right) ; \nu_{1+n_{1}+j_{2}}=n_{2}-j_{2}\left(j_{2}=0,1, \ldots, \overline{n_{2}-1}\right)$ u. s. f., so erkennt man leicht die Richtigkeit der Gleichung

$$
y=\frac{p^{\varrho=1} \sum_{1}^{g}(2 \varrho-1) \alpha_{\varrho}}{n_{1} ! \ldots n_{r} !} \prod_{\varrho=1}^{\varrho=r}\left\{\left(1-\frac{1}{p^{n_{o}}}\right) \ldots\left(1-\frac{1}{p}\right)\right\},
$$

welche nach Einführung der Größen $\overline{\alpha_{j}}$ das folgende Theorem ergibt: Besitzt eine Abel'sche GruppeH, deren Grad eine Primzahlpotenz $p^{\pi}$ ist, ein Basissystem, welches aus $n_{1}$ Elementen vom Grade $p^{a_{1}}$, aus $n_{2}$ Elementen vom Grade $p^{\alpha_{2}}, \ldots$ aus $n_{r}$ Elementen (4) vom Grade $p^{\alpha_{r}}$ zusammengesetzt ist, so gibtes genau

$$
\prod_{j=1}^{j=r} \frac{p^{n_{j}\left(2 \overline{n_{1}+\cdots+n_{j}}-n_{j}\right) a_{j}}\left(1-\frac{1}{p^{n_{j}}}\right) \cdots\left(1-\frac{1}{p}\right)}{n_{j} !}
$$

verschiedene Basissysteme dieser Gruppe $\left(\alpha_{1}>\alpha_{2}>\ldots>\alpha_{r}\right)$. 
Wenn man bedenkt, dass die reguläre Gruppe $1, A_{j}, \ldots, A_{j}^{p^{\alpha_{j}-1}}$ gerade $\varphi\left(p^{\alpha_{j}}\right)$ Elemente vom Grade $p^{\alpha_{j}}$ enthält, so findet man auf Grund des Satzes (4) für die Anzahl der verschiedenen Systeme regalärer Gruppen, aus denen sich die Gruppe $\mathrm{H}$ componieren läsșt, ohne Mühe den Ausdruck

$$
\prod_{j=1}^{j=r} \frac{p^{n_{j}\left(2 \overline{n_{1}+\cdots+n_{j}}-n_{j}-1\right) \alpha_{j}}}{n_{j} !} \frac{\left(1-\frac{1}{p^{n_{j}}}\right)}{1-\frac{1}{p}} \cdots \frac{1-\frac{1}{p^{2}}}{1-\frac{1}{p}} \cdot \frac{1-\frac{1}{p}}{1-\frac{1}{p}} .
$$

Dieses Product wird speciell für $r=1$ und $n_{1}=1$ selbst gleich der Einheit, woraus man schließt, dass jede reguläre Gruppe, die ja von selbst eine Abel'sche ist, nicht weiter in reguläre Gruppen decomponabel sein kann, sobald ihr Grad gleich einer Potenz einer Primzahl ist; ein Satz, der sich übrigens auch auf anderem Wege sehr leicht nachweisen lässt.

Selbstverständlich kann der oben geführte Existenzbeweis eines Basissystemes wörtlich auf eine jede A bel'sche Gruppe angewendet werden, welche dadurch charakterisiert erscheint, dass alle ihre Elemente Gradzahlen besitzen, die Potenzen einer und derselben Primzahl sind, woraus folgt, dass der Grad einer jeden solchen Gruppe selbst eine Potenz dieser Primzahl sein muss.

\section{Nr. 3. Über die Untergruppen einer Abel'schen Gruppe, deren Grad eine Primzahlpotenz ist.}

Da jede Untergruppe einer Abel'schen Gruppe H, deren Grad eine Primzahlpotenz $p^{\text {tr }}$ ist, wieder eine Gruppe der gleichen Beschaffenheit vorstellt, muss auch sie nach dem Satze (1) der vorigen Nr. ein Basissystem besitzen. Naturgemäb kann dieses Basissystem, wenn man den Satz (3) derselben Nr. beriücksichtigt, einen Eintheilungsgrund für alle Untergruppen eines vorgegebenen Grades $p^{\beta}$ abgeben, indem man alle jene Untergruppen dieses Grades als zu einer und derselben Klasse gehörig betrachtet, welche Basissysteme von derselben Elementenzahl und den gleichen Gradzahlen besitzen.

Haben äie Basiselemente $B_{1}, \ldots, B_{r}$ einer bestimmten solchen Untergruppe $\overline{\mathrm{H}}$ die nicht zunehmenden Gradzablen $p^{\beta_{1}}$, bezw. $p^{\beta_{2}}, \ldots$, bezw. $p^{\beta_{r}}\left(\beta_{r}>0\right)$, so soll demgemäh gesagt werden, die Gruppe $\overline{\mathrm{H}}$ gehörezur Klasse $\left(\beta_{1}, \ldots, \beta_{r}\right)$.

Da die Gleichung $\beta_{1}+\beta_{2}+\cdots+\beta_{r}=\beta$ bestehen muss, braucht man, um alle die die einzelnen Klassen charakterisierenden Zahlsysteme $\beta_{1}, \ldots, \beta_{x}$ zu erhalten, bloß die Zahl $\beta$ auf alle möglichen Arten in ganzzahlige, positive Addenden zu zerlegen; und die Frage nach der Gesammtzahl der Untergruppen vom Grade $p^{\beta}$ 
kommt somit auf die Bestimmung der Anzahl der einer Klasse $\left(\beta_{1}, \ldots, \beta_{r}\right)$ angehörigen Untergruppen zurück.

Um diese letztere Anzahl zu ermitteln, kann man einen ähnlichen Weg, wie in der vorigen Nr. bei der Bestimmung der Anzahl der Basissyteme einer A bel'schen Gruppe einschlagen.

Zuvor möge aber eine auch für das Folgende wichtige, abkürzende Ausdrucksweise eingeführt werden:

Bezeichnet man mit $L$ und $M$ zwei beliebige Individuen irgend einer Gruppe $\Gamma$, so soll ein jedes in $\Gamma$ enthaltene Element, dessen $\delta^{\text {te }}$ Potenz mit $L$ multipliciert zum Resultat $M$ gibt, eine $W$ u rze 1 der Gleichung

$$
L X^{\delta}=M
$$

im Gebiete $\Gamma$ genannt werden.

Weil das $p^{\text {te }}$ Basiselement $B_{\varrho}$ der der Klasse $\left(\beta_{1}, \ldots, \beta_{r}\right)$ angehörigen Untergruppe $\overline{\mathrm{H}}$ ein solches Element vom Grade $p^{\beta}$ innerhalb der Gruppe $\mathbf{H}$ vorstellt, welches erst auf die $\left(p^{\beta} \rho\right)^{\text {te }}$ Potenz erhoben und nicht früher in dem Systeme

$\left(\mathrm{E}_{\varrho-1}\right) \quad B_{1}^{k_{1}} \ldots B_{\varrho-1}^{k_{\varrho-1}} \quad\left(\begin{array}{c}k_{i}=0,1, \ldots,\left(p^{\beta_{i}}-1\right) \\ i=1,2, \ldots, p-1\end{array}\right)$

vorkommt, lässt sich dasselbe als eine derjenigen Wurzeln der Gleichung

$$
X^{p^{\beta_{Q}}}=1
$$

im Gebiete $\mathrm{H}$ charakterisieren, welche keine der Gleichungen

$$
X^{p^{\beta_{\varrho}-1}}=C_{i} \quad\left(i=1,2, \ldots, p^{\varrho-1}\right)
$$

befriedigen, wo für $C_{1}, C_{2}, \ldots$ der Reihe nach alle Wurzeln der Gleichung

$$
X^{p}=1
$$

im Gebiete $\left(E_{o-1}\right)$ za setzen sind.

Die Zahl der Wurzeln der Gleichung (I) wurde bereits in der vorigen Nr. ermittelt; sie ist identisch mit der Anzahl $\mathfrak{A}$ derjenigen Elemente von $\mathrm{H}$, deren Gradzahlen den Exponenten $p^{\beta} \varrho$ nicht übertreffen und betrïgt somit

$$
p^{j_{Q} \beta_{Q}+\alpha_{j}+1}+\cdots+\alpha_{s},
$$

wenn die Gruppe $\mathrm{H}$ zur Klasse $\left(\alpha_{1}, \ldots, \alpha_{s}\right)$ gehört und $j_{0}$ der Elemente $\alpha_{i}(i=1,2, \ldots, s)$ nicht kleiner als $\beta_{e}$ sind. 
Was die Gleichungen (II) anlangt, beachte man, dass jedes Element $C_{i}$ als Wurzel der Gleichung (III) im Gebiete $\left(\mathrm{E}_{\varrho-1}\right)$ die Form $B_{1}^{k_{1} p^{\beta_{2}-1}} \ldots B_{\varrho-1}^{k_{o-1} p^{\beta_{g}-1-1}}$ besitzen, sich also in die Gestalt.

$$
\left(B_{1}^{k_{1} p^{\beta_{1}-\beta_{Q}}} \ldots B_{Q}^{k_{Q-1}-1} p^{\beta_{Q}-1-\beta_{Q}}\right)^{p^{\beta_{0}-1}}
$$

bringen lassen muss, wo unter $k_{1}, \ldots, k_{o-1}$ ganze Zahlen zu verstehen sind. Daraus folgt, dass jede Gleichung (II) gewiss eine Wurzel und damit entsprechend der Gleichung $X^{p^{\beta} e^{-1}}=1$, wie leicht nachzuweisen ist, $\left.{ }^{1}\right)$ im Gebiete $\mathrm{H}$ genau $p^{j_{Q}\left(\beta_{e}-1\right)+a_{j_{0}}+1+\ldots+\alpha_{s}}$ Wurzeln besitzt.

$\mathrm{Da}$ jedoch $p^{a^{-1}}$ solcher Gleichungen auftreten, beträgt die Gesammtzahl $\mathfrak{B}$ der Wurzeln aller Gleichungen (II)

$$
p^{j_{Q} \beta_{g}+\alpha_{j_{g}}+1+\cdots+\alpha_{s}-\left(j_{e}-\bar{Q}-1\right)} \text {. }
$$

Setzt man also die Existenz der Gruppe $\left(\mathrm{E}_{e-1}\right)$ mit ảen Basiselementen $B_{1}, \ldots, B_{\rho-1}$ voraus, so lehrt die Vergleichung derbeiden Zahlen $\mathfrak{A}$ und $\mathfrak{Q} \mathfrak{B}$, dass dann und nur dann auch Elemente $B_{e}$ existieren, wenn die Ungleichung

$$
j_{o}>p-1
$$

erfüllt wird; und zwar kann man dann das Basiselement $B_{\varrho}$ aut

erlei Arten wählen.

$$
p^{j_{Q} \beta_{Q}+\alpha_{j_{Q}}+1+\cdots+\alpha_{s}}\left(1-\frac{1}{p^{j_{\varrho}-\bar{\varrho}-1}}\right)
$$

Werden die Gleichungen $\alpha_{1} \geqq \alpha_{2} \geqq \cdots \geqq a_{s}$ berücksichtigt, so ergibt sich, dass die Beziehung $j_{Q}>\overline{p-1}$ stets erfüllt ist, sobald die Relation

$$
\beta_{e} \leqq \alpha_{e}
$$

besteht, und dass umgekehrt diese letztere Beziehung eine nothwendige Folge der ersteren ist.

Man kann somit das folgende Theorem aussprechen:

Eine der Klasse $\left(\alpha_{1}, \ldots, \alpha_{s}\right)$ angehörige Gruppe hat dann und nur dann Untergruppen von der (1) Klasse $\left(\beta_{1}, \ldots, \beta_{r}\right)$, wenn die $r$ Bedingungen

erfüllt sind.

$$
\beta_{e} \leqq \alpha_{Q} \quad\left(\begin{array}{c}
p=1,2, \ldots, r \\
r \leqq s
\end{array}\right)
$$

1) Man vergleiche die nächste Nr. 
Aus diesem Satze folgt sofort die Existenz von Untergruppen eines jeden Grades $p^{\beta}$, der kleiner als der Grad $p^{x t}$ der Gruppe $H$ ist.

Die Annahme nämlich, dass der Exponent $\beta$ kleiner als $\alpha_{1}$, oder gleich einer der Summen $\alpha_{1}+\cdots+\alpha_{i}(i=1,2, \ldots, \overline{\rho-1})$ sei, erledigt sich von selbst. Andernfalls jedoch kann man die Zahl $r$ der Art bestimmen, dass die Beziehung

$$
\alpha_{1}+\cdots+\alpha_{r-1}<\beta<\alpha_{1}+\cdots+\alpha_{r}
$$

besteht, dass also nach dem Satze (1) Untergruppen der Klasse $\left(\alpha_{1}, \ldots, \alpha_{r-1}, \beta-\sum_{i=1}^{r-1} \alpha_{i}\right)$ existieren. Es leuchtet dies übrigens auch ohne Weiteres ein.

Erfüllen die Zahlen $\beta_{i}(i=1,2, \ldots, r)$ thatsächlich die durch den Satz (1) festgestellten Bedingungen, so wird nach dem Ausdrucke (IV) die Gesammtzahl der möglichen Basissysteme von Gruppen der Klasse $\left(\beta_{1}, \ldots, \beta_{r}\right)$ durch das Product

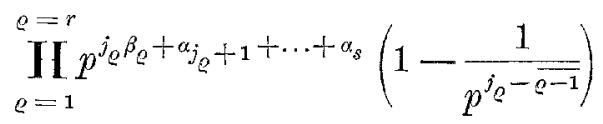

bestimmt erscheinen.

Sieht man von der Reihenfolge der Elemente innerhalb eines jeden Basissystemes ab, so erhält man, falls $m_{1}$ Zahlen der Reihe $\beta_{1}, \ldots, \beta_{r}$ gleich $\gamma_{1}, \ldots, m_{t}$ Zablen der Reihe $\beta_{1}, \ldots, \beta_{r}$ gleich $\gamma_{t}$ $\left(\gamma_{1}>\gamma_{2}>\cdots>\gamma_{t}\right)$ and dementsprechend auch $m_{1}$ Zahlen der Reihe $j_{1}, \ldots, j_{r}$ gleich $h_{1}, \ldots, m_{t}$ Zahlen der Reihe $j_{1}, \ldots, j_{r}$ gleich $h_{t}$ sind, als Anzahl der verschiedenen Basissysteme den Ausdruck

$$
\begin{aligned}
& \prod_{\varrho=1}^{\varrho=t} \frac{1}{m_{\varrho} !} p^{m_{\varrho}\left(h_{\varrho} \gamma_{\varrho}+a_{h_{\varrho}}+1+\cdots+\alpha_{s}\right)}\left(1-\frac{1}{p^{h_{\varrho}-\overline{m_{1}+m_{2}+\cdots+1 a_{\varrho}}+m_{\varrho}}}\right) \cdots \\
& \cdots\left(1-\frac{1}{p^{h_{e}-\overline{m_{1}+m_{2}+\ldots+m_{Q}}+1}}\right) .
\end{aligned}
$$

Da jedoch nach dem Satze (4) der vorigen Nr. je

$$
\prod_{\varrho=1}^{\varrho=t} \frac{p^{m_{\varrho}\left(2\left(m_{1}+\ldots+m_{\varrho}\right)-m_{\varrho}\right) r_{\varrho}}}{m_{\varrho} !}\left(1-\frac{1}{p^{m_{\varrho}}}\right) \cdots\left(1-\frac{1}{p}\right)
$$

verschiedene Basissysteme einer und derselben Gruppe angehören, gelangt man zu dem folgenden Theorem: 
Eine der Klasse $\left(\alpha_{1}, \ldots, \alpha_{s}\right)$ angehörige Abelsche Gruppe besitzt genau

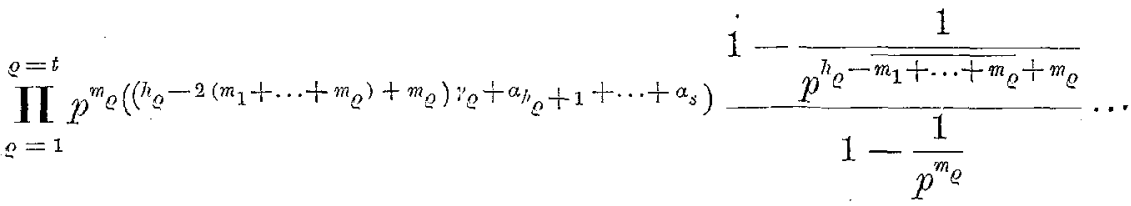

$$
\begin{aligned}
& \cdots \frac{1-\frac{1}{p^{h_{e}-\overline{m_{1}}+\cdots+\overline{m_{e}}+1}}}{1-\frac{1}{p}}
\end{aligned}
$$

Untergruppen mit $m_{1}$ Basiselementen vom Grade $p^{\nu_{t}}, \ldots, m_{t}$ Basiselementen vom Grade $p^{\gamma_{t}}$, vorausgesetzt, dass die Bedingungen

$$
\gamma_{Q} \leqq \alpha_{m_{1}+\ldots+m_{e}} \text { für } p=1,2, \ldots, t
$$

erfüllt sind und unter $h_{\varrho}$ die Anzabl derjenigen Zahlen der Reihe $\alpha_{1}, \ldots, \alpha_{s}$ verstanden wird, welche nicht kleiner als $\gamma_{0}$ sind $\left(\gamma_{1}>\gamma_{2}>\cdots>\gamma_{t}\right)$.

Dieser Satz bestimmt die Anzahl $\mathfrak{A F}_{\beta_{1}}, \ldots, \beta_{r}$ derjenigen Untergruppeu der Gruppe $\mathrm{H}$, welche zur Klasse $\left(\beta_{1}, \ldots, \beta_{r}\right)$ gebören; um also die Gesammtzahl der Untergruppen rom Grade $p^{\beta}=p^{\beta_{1}+\cdots+\beta_{r}} \mathrm{zu}$ erhalten, hat man die Summe

$$
\sum_{\beta_{1}+\ldots+\beta_{e}=\beta} \mathfrak{A}_{\beta_{1}, \ldots, \beta_{e}}
$$

zu bilden, welche sich auf alle jene ganzzahligen, positiven Darstellungen $\beta_{1}+\cdots+\beta_{o}$ der Zahl $\beta$ zu erstrecken hat, die den Bedingungen des Theoremes (1) Genüge leisten. Satz :

Setzt man speciell $s=1$, so erhält man den folgenden

Jede reguläre Gruppe vom Grade $p^{-\tau}$ enthält nur eine Untergruppe vom Grade $p^{\beta}(\beta<\pi)$.

Letztere kann leicht angegeben werden und ist selbst regulär. Als Illustration der vorstehenden Betrachtungen möge das folgende Beispiel dienen:

Die Gruppe $\mathrm{H}$ vom Grade $p^{\pi}$ gehöre der Klasse $\left(\alpha_{1}, \ldots, \alpha_{s}\right)$ an, deren Zahlen $\alpha_{1}, \ldots, \alpha_{s}$ die Bedingungen $\alpha_{i}>\alpha_{i+1}+1$ $\left(\begin{array}{c}\alpha_{s}>1 \\ i=1, \ldots, s-1\end{array}\right)$ erfüllen. Es soll die Anzahl der Untergruppen von $\mathrm{H}$ vom Grade $p^{\pi-1}$ bestimmt werden. 
In diesem Falle sind, wie eine einfache Überlegung lehrt, nur die Zerlegungen $\alpha_{1}+\cdots+\alpha_{i-1}+\left(\alpha_{i}-1\right)+\alpha_{i+1}+\cdots+\alpha_{s}$ $(i=1,2, \ldots, s)$ der Zahl $\beta=\pi-1$ brauchbar. Man siebt, dass unter dieser Annahme stets alle Zahlen $m_{\rho}$ gleich 1 sind, während $h_{e}$ den Wert $p$ erhält; es besteht somit die Gleichung. $\mathfrak{H}_{a_{1}, \ldots, \alpha_{i}-1, \ldots, \alpha_{s}}=p^{i-1}$ and die gesuchte Anzahl wird gleich $\frac{p^{s}-1}{p-1}$.

Schließlich mag noch darauf hingewiesen werden, dass auf Grund des Theoremes (2) der vorigen Nr. leicht die Anzahl der regulären Untergruppen vom Grade $p^{\sigma}$ der Gruppe $\mathrm{H}$ angegeben werden kann.

Bezeichnet man nämlich mit $A$ ein Element vom Grade $p^{\sigma}$, so enthält die Reihe $1, \ldots, A^{p^{\sigma}-1}$ genau $\varphi\left(p^{\sigma}\right)$ Elemente rom selben Grad und keines dieser Elemente kommt in einer andern Untergruppe vom Grade $p^{\sigma}$ vor. D. h.:

Die Anzahlder reguaren Untergruppenvom Grade $p^{\sigma}$ einer Abel'schen Gruppe der Klasse $\left(a_{1}, \ldots, \alpha_{s}\right)$ ist gleich

$$
\frac{p^{i \sigma+a_{i+1}+\ldots+\alpha_{s}}\left(1-\frac{1}{p^{i}}\right)}{\varphi\left(p^{\sigma}\right)}
$$

wenn mit $i$ die Anzahl der Zahlen $\alpha_{j}(j=1,2, \ldots, s)$ bezeichnet wird, welche nicht kleiner als $\sigma$ sind.

Da jede Gruppe von einem Primzahlgrad regulär ist, erhält man aus diesem Satze, indem man $\sigma=1$ setzt, in Ửbereinstimmung: mit dem Theoreme (2) den folgenden:

Die betrachtete Abel'sche Gruppe hat genau $\frac{p^{s}-1}{p-1}$ Untergruppen vom Grade' $p$.

\section{Nr. 4. Uber das Basissystem einer Abel'schen Gruppe, deren Grad eine beliebig zusammengesetzte Zahl ist.}

Es sei eine Abel'sche Gruppe H rom Grade $n$ gegeben, wobei die Zahl $n$ in ihre Primfactoren zerlegt gleich dem Producte $i=\hbar$

ПI $p_{i}^{\pi_{i}}$ angenommen wird.

$i=I$ Ohne Weiteres lässt sich die Existenz von zweierlei Arten von Untergruppen innerhalb der Gruppe H feststellen: 
Es bildet erstens die Gesammtheit der Wurzeln einer jeden Gleichung

$$
X^{\delta}=1
$$

im Gebiete $\mathrm{H}$ stets eine Unteruppe $\mathrm{H}_{\delta}$ von $\mathrm{H}$, weil aus den beiden Beziehungen $A_{1}^{\delta}=1$ and $A_{2}^{\delta}=1$ vermöge des commutativen Gesetzes auch die Relation $\left(A_{1} A_{2}\right)^{\delta}=1$ folgt.

Um ebenso die zweite Art von Untergruppen einfach zu charakterisieren, ist es vortheilhaft, die folgende Ausdrucksweise einzuführen:

Im Falle, als die Gleichung

$$
X^{\delta}=M
$$

im Gebiete $\mathrm{H}$ eine Wurzel besitzt, soll gesagt werden, das Element $M$ sei eine $\delta^{\text {te }}$ Potenz innerhalb der Gruppe $\mathrm{H}$.

Da neben den Gleichungen $S_{1}^{\delta}=A_{1}$ und $S_{2}^{\delta}=A_{2}$ auch die Gleichung $\left(S_{1} S_{2}\right)^{\delta}=A_{1} A_{2}$ besteht, stellt der Inbegriff aller $\delta^{\text {ten }}$

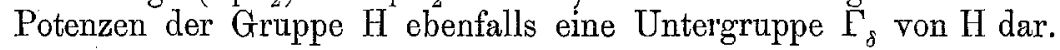

Weiters ist klar, dass außer dem Elemente $S_{1}$ auch jedes Element des Systemes

die Gleichung

$$
\mathrm{H}_{\delta} S_{1}
$$

$$
X^{\delta}=A_{1}
$$

befriedigt und dass dies die einzigen Individuen der Gruppe $\mathrm{H}$ sind, welche diese Eigenschaft besitzen.

Denn aus der Gleichung $S^{\delta}=A_{1}$, d. h. $S^{\delta}=S_{1}^{\delta}$, folgt die Beziehung $\left(S S_{1}^{-1}\right)^{\delta}=1$.

Bezeichnet man mit

$$
A_{0}, A_{1}, \ldots, A_{k-1} \quad\left(A_{0}=1\right)
$$

alle verschiedenen $\delta^{\text {ten }}$ Potenzen der Gruppe $H$ und mit $S_{i}$ irgend eine Wurzel der Gleichung:

$$
X^{\delta}=A_{i}(i=0,1, \ldots, \overline{k-1}),
$$

so stellen entsprechend der eben angeführten Thatsache die Systeme

$$
\mathrm{H}_{\delta} ; \mathrm{H}_{\delta} S_{1} ; \ldots ; \mathrm{H}_{\delta} S_{k-1}
$$

successive alle Wurzeln aller Gleichungen (I) dar. Die Reihe (î) enthält mithin alle Elemente der Gruppe $H$ und ein jedes nur einmal, weil ein jedes Individuum derselben eine und nur eine der Gleichungen (I) befriedigt. 
Man schließt daraus den Satz, dass das Product der Gradzahlen der beiden Gruppen $\mathrm{H}_{\delta}$ und $\Gamma_{\delta}$ gleich dem Grade von $\mathrm{H}$ ist. Wählt man nun speeiell für $\delta$ einen solchen Theiler von $n$, dass die beiden Zahlen $\frac{n}{\delta}$ und $\delta$ theilerfremd sind, so können sich keine zwei $\delta^{\text {ten }}$ Potenzen $A_{m}$ und $A_{e}$ in einem und demselben Systeme

$$
\mathrm{H}_{\delta} S_{i}\left(i=0,1, \ldots, \overline{k-1} ; S_{0}=1\right)
$$

des Schemas (î) vorfinden.

Es bestünde ja sonst die Gleichung $A_{m}^{\delta}=A_{e}^{\delta}$, aus welcher die Beziehung $\left(S_{m} S_{e}^{-1}\right)^{\delta^{2}}=1$ folgen würde. Dann müsste der Grad des Productes $S_{m} S_{e}^{-1}$, welcher von selbst Theiler von $n$ ist, auch Theiler von. $\delta^{2}$, also auch von $\delta$ selbst sein. Die Relation $\left(S_{m} S_{e}^{-1}\right)^{\delta}=1$ würde aber den Widerspruch $A_{m}=A_{e}$ nach sich ziehen.

Es findet sich somit in jedem Systeme $\mathrm{H}_{\delta} S_{i}(i=0, \ldots, \overline{k-1})$ eine $\delta^{\text {te }}$ Potenz vor; und da es gleichgiltig ist, welches Element des Systemes $\mathrm{H}_{\delta} S_{i}$ an die Stelle des Elementes $S_{i}$ gesetzt wird, kann speciell für dasselbe die eben hervorgehobene $\delta^{\text {te }}$ Potenz genommen werden.

Man erkennt alsdann, dass die Gruppe $\mathrm{H}$ als durch Composition der beiden Untergruppen $\mathrm{H}_{\delta}$ und $\Gamma_{\delta}$ erzeugt, gedacht werden kann.

Es ist klar, dass die Untergruppe $\Gamma_{\delta}$ kein Element enthält, dessen Grad durch einen Divisor $\tau$ von $\delta$ theilbar ist.

Denn käme in der Gruppe $\Gamma_{\delta}$ das Element $A$ vom Grade $\mu \tau$ vor, so enthielte dieselbe Gruppe auch die Potenz $A^{u}$ vom Grade $\tau$, was unmöglich der Fall sein kann, weil das Element $A^{\mu}$ als Wurzel der Gleichung $X^{\delta}=1$ der Gruppe $\mathrm{H}_{\delta}$ angehört.

Es lässt sich also, indem man speciell $\delta=p_{1}^{\tau_{1}}$ setzt, die Abel'sche Gruppe

$\mathrm{H}$

in die beiden Componenten

$$
\mathrm{H}_{p_{1} \pi_{1}} \text { und } \Gamma_{p_{1} \pi_{1}}
$$

zerfällen, wobei die letztere nur Elemente umfasst, deren Gradzahlen den Primtheiler $p_{1}$ nicht besitzen.

Wendet man dieselben Betrachtungen auf die Abel'sche Gruppe $\Gamma_{p_{1}} \pi_{1}$ an, so lässt sich auch diese in zwei Untergruppen der Art zerlegen, dass die eine von ihnen bloß Elemente umfasst, deren 
Gradzahlen Potenzen von $p_{2}$ sind, während die andere kein Element enthält, dessen Grad den Primfactor $p_{2}$ besitzt.

Indem man nun weiters die nämlichen Überlegungen wieder auf diese letztere Untergruppe anwendet u. s. f., gelangt man schlieblich zu einer Untergruppe, die bloß solche Elemente umfasst, deren Gradzahlen den einzigen Primtheiler $p_{h}$ besitzen.

Man hat demnach die Gruppe $\mathrm{H}$ in lauter Untergruppen zerlegt, deren Gradzahlen gemäß einer Bemerkung am Schlusse der Nr. 2 durch Primzahlpotenzen dargestellt werden, deren Eigenschaften also in den beiden vorangehenden Nummern eingehend untersucht worden sind.

Da die Gradzahlen der zu componierenden Gruppen untereinander multipliciert den Grad von $\mathrm{H}$ ergeben müssen, können sie nur der Reihe nach beziehungsweise die Werte $p_{1}^{\pi_{1}}, p_{2}^{\pi_{2}}, \ldots, p_{h}^{\pi_{h}}$. besitzen, woraus ohne Mühe der folgende Satz erschlossen werden kann :

Die Gruppe H lässt sich als Resultat der

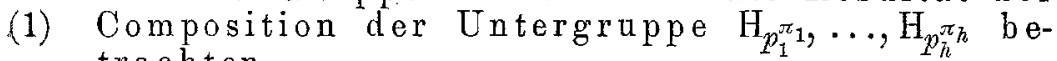
trachten.

Berücksichtigt man überdies den. Satz (1) der Nr. 2, so kann man das folgende von Kronecker ${ }^{1}$ ) bewiesene, fundamentale Theorem ausprechen:

Es gibt innerhalb einer jeden Abel'schen Gruppe H Elemente $B_{i}$ vom Grade $n_{i}(i=1,2, \ldots, s)$ der Art, dass ein jedes Element der Gruppe $\mathrm{H}$ in der Form

$$
\prod_{i=1}^{i=s} B_{i}^{u_{i}}\left(\mu_{i}=0,1, \ldots, \overline{n_{i}-1}\right)
$$

und zwar jedes nur einmal enthalten ist.

Der Inbegriff solcher Elemente $B_{1}, \ldots, B_{s}$ wird ein Basissystem der Abel'schen Gruppe $\mathrm{H}$ genannt.

Die Gradzahlen $n_{i}$ der Basiselemente können beliebige ganze Zahlen sein; sie werden hier absichtlich mit Rücksicht auf die Anwendungen (vergl. Abschnitt III) nicht gleich Primzahlpotenzen vorausgesetzt, wie dies nach dem eingeschlagenen Beweisverfahren möglich gewesen wäre.

Die Anzahl der Basiselemente ist in dem hier behandelten allgemeinen Falle durchaus nicht constant, wie dies nach Satz.(3) der Nr. 2 für A bel'sche Gruppen, deren Gradzahlen Primzahlpotenzen sind, gilt.

1) Monatsber. d. Berliner Akad, vom Dec. 1870; Werke I., p. 278.

Vergl. auch Weber, Ellipt. Fet. u. allg. Z,, 1891, p. 178. 
Ist nämlich einerseits der Grad $n_{i}$ des Basiselementes $B_{i}$ gleich dem Producte $n_{i}^{\prime} n_{i}^{\prime \prime}$, wo $n_{i}^{\prime}$ und $n_{i}^{\prime \prime}$ zueinander relativ prim vorausgesetzt werden, so kann das Basiselement $B_{i}$ als äquivalent den beiden Basiselementen $B_{i}^{n_{i}^{\prime}}$ und $B_{i}^{n_{i}^{\prime \prime}}$ zusammengenommen betrachtet werden, indem die reguläre Gruppe

$$
1, B_{i}, \ldots, B_{i}^{n_{i}-1}
$$

durch Composition der beiden regulären Untergruppen

$$
1, B_{i}^{n_{i}^{\prime}}, \ldots, B_{i}^{n_{i}^{\prime}\left(n_{i}^{\prime \prime}-1\right)} \text { und } 1, B_{i}^{n_{i}^{\prime \prime}}, \ldots, B_{i}^{n_{i}^{\prime \prime}\left(n_{i}^{\prime}-1\right)}
$$

erzeugt werden kann.

Andererseits ist klar, dass die beiden Gruppen $B_{i}^{\mu_{j}}$ $\left(\mu_{j}=0, \ldots, \overline{n_{i}-1}\right)$ und $B_{k}^{\mu_{j}}\left(\mu_{j}=0, \ldots, \overline{n_{k}-1}\right)$, falls ihre Gradzablen $n_{i}$ und $n_{k}$ theilerfremd sind, mit einander componiert die eine Gruppe $\left(B_{i} B_{k}\right)^{\mu_{j}}\left(\mu_{j}=0, \ldots, \overline{n_{i} n_{k}-1}\right)$ ergeben, dass also die beiden Basiselemente $B_{i}$ und $B_{k}$ durch das eine $\left(B_{i} B_{k}\right)$ ersetzbar sind.

Während die erstere Bemerkung die schon bekannte Thatsache bestätigt, dass die Basiselemente der Art gewählt werden können, dass ihre Gradzahlen Primzahlpotenzen sind, lehrt die letztere, dass diese $\mathrm{Wahl}$ auch so getroffen werden kann, dass keine zwei Gradzahlen theilerfremd sind, bezw. dass jeder Grad ein. Multiplum des folgenden ist. ${ }^{1}$ )

Im Falle, dass je zwei der Gradzahlen $n_{i}$ zueinander theilerfremd sind, ist die Gruppe regulär.

Unter allen Basissystemen sind diejenigen, welche die größte Zahl von Elementen besitzen, ausgezeichnet. Auf sie hat ja auch der oben angegebene Existenzbeweis geführt. Ihre Anzahl lässt. sich mit Hilfe des Satzes (4) der Nr. 2 leicht angeben.

Da die Gruppe $\mathrm{H}$ durch Composition aus den Untergruppen $\mathrm{H}_{p_{i}^{\pi_{i}}}(i=1, \ldots, h)$ hervorgeht, sind die Classen $\left(\alpha_{1}^{(i)}, \ldots, \alpha_{r_{i}}^{(i)}\right)$ $(i=1, \ldots, h)$, zu denen bezw. dic angeführten Componenten gehören, auch als charakteristisch für die Gruppe $\mathrm{H} z u$ betrachten; und es soll gesagt werden, die Gruppe $H$ gehöre zur Klasse $\left(\alpha_{1}^{(i)}, \ldots, \alpha_{r_{i}}^{(i)}\right)(i=1,2, \ldots, h)$.

Die Zahlen $\alpha_{1}^{(i)}, \ldots, \alpha_{r_{i}}^{(i)}$ sind natürlich nichts anderes, als die Exponenten derjenigen Potenzen von $p_{i}(i=1,2, \ldots, h)$, welche man erhält, sobald man die Gradzahlen $n_{1}, \ldots, n_{s}$ der Reihe nach in ihre Primfactoren zerlegt.

1) Vergl. Kronecker l. c. 
Nr. s. Über die Untergruppen einer allgemeinen Abel'schen Gruppe.

Behält man die eben eingeführte Bezeichnungsweise bei und versteht man unter

$$
j_{1}, j_{2}, \ldots, j_{t}
$$

irgend $t$ der Reihe $1, \ldots, h$ angehörige Zahlen, so gilt, da jede Untergruppe einer Abel'schen Gruppe selbst eine solche ist, gemäb dem Satze (1) der vorigen Nr. das folgende Theorem:

Eine jede der Gruppe H angehörige Untergruppe $\overline{\mathrm{H}}$ von dem zusammengesetzten Grade II $p_{j_{i}}^{k_{j}}$ kann als durch Composition von $t$ bestimmten $i=1$

Untergruppen $\overline{\mathrm{E}}_{p_{j_{i}}}^{k_{j_{i}}}$ mit den bezüglichen Gradzahlen $p_{j_{i}}^{k_{j_{i}}}(i=1, \ldots, t)$ entstanden gedachtwerden. $\left(k_{j_{i}} \leqq \pi_{j_{i}}\right)$ Es bedarf kaum der Erwähnung, dass die Gruppen $\overline{\mathrm{H}}_{p_{j_{i}}^{k_{j}}}$ selbst Untergruppen der Systeme $\mathrm{H}_{p_{j_{i}}^{\tau} j_{i}}$ darstellen.

Auf Grund des Theoremes (1) der Nr. 3 erhält man also den folgenden Satz:

Eine Abel'sche Gruppeder Klasse $\left(\alpha_{1}^{(i)}, \ldots, \alpha_{r_{i}}^{(i)}\right)$ $(i=1,2, \ldots, h)$ besitztdann und nurdannzur Klasse $\left(\beta_{1}^{\left(j_{i}\right)}, \ldots, \beta_{r_{i}^{\prime}}^{\left(j_{i}\right)}\right)(i=1, \ldots, t)$ gehörige Untergruppen, wenn die $\sum_{i=1}^{t} r_{j_{i}}^{r}$ Bedingungen

$$
\beta_{k}^{\left(j_{i}\right)} \leqq \alpha_{k}^{\left(j_{i}\right)}\left(\begin{array}{c}
k=1,2, \ldots, r_{i}^{\prime} \\
i=1,2, \ldots, t
\end{array}\right)
$$

erfüllt sind. $\left(r_{j_{i}} \geqq r_{j_{i}}^{\prime}\right)$.

Wird diesen Forderungen Genüge geleistet, so lässt sich die Zahl der möglichen Untergruppen der betrachteten Klasse auf Grund des Theoremes (2) der angeführten Nummer leicht angeben.

Ebenso lehren die auf dieses Theorem folgenden Bemerkungen derselben Nummer, dass die Gesammtzahl der Untergruppen des gegebenen Grades $\prod_{i=1}^{i=t} p_{j_{i}}^{k_{j_{i}}}$ dureh das Product

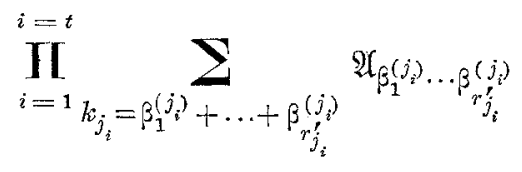


ausgedrückt werden kann, wo unter $\mathfrak{A}_{\beta_{1}^{\left(j_{i}\right)} \ldots \beta_{r_{j_{i}}}^{\left(j_{j}\right)}}$ die Anzahl der Untergruppen der Klasse $\left(\beta_{1}^{\left(j_{i}\right)}, \ldots, \beta_{r_{j_{i}}^{\prime}}^{\left(j_{i}\right)}\right)$ zu verstehen ist und sich die Summe auf alle die Bedingungen des Theoremes (2) erfüllenden positiven ganzzahligen Systeme $\beta_{1}^{\left(j_{i}\right)}, \ldots, \beta_{r_{j}^{\prime}}^{\left(j_{i}\right)}$ zu erstrecken hat.

Es ist klar, dass stets wenigstens eine Untergruppe der Gruppe $H$ von jedem Grade existiert, welcher den Grad von. $\mathrm{H}$ theilt.

Im Falle, als die Exponenten $k_{j_{i}}$ gleich den Zahlen $\pi_{j_{i}}$ für $i=1, \underset{i=t}{2, \ldots}, t$ sind, gibt es natürlich nur eine Untergruppe vom Grade $\prod_{i=1} p_{j_{i}}^{k_{j_{i}}}$ weil dann die systeme $\overline{\mathrm{H}}_{p_{j_{i}}^{\eta_{j}}}$ mit den Gruppen $\mathrm{H}_{p_{j_{i}}^{\pi_{j}}}$ identisch werden.

Was die der Gruppe H angehörigen regulären Untergruppen von einem zusammengesetzten Grade betrifft, beachte man, dass sich dieselben wieder in reguläre Gruppen, deren Grade aber Primzahlenpotenzen sind, zerlegen lassen, wie dies schon in der vorigen $\mathrm{Nr}$. ausgeführt worden ist.

Man erbält also unter Berücksichtigung des Satzes (4) der Nr. 3 das tolgende Theorem:

Gehört eine Abel'sche Gruppe der Klasse $\left(\alpha_{1}^{(i)}, \ldots, \alpha_{r_{i}}^{(i)}\right)(i=1, \ldots, h)$ an, so besitzt sie

$$
\frac{1}{\varphi(\delta)} \prod_{i=1}^{i=t} p_{j_{i}}^{l_{j} j_{j}}+\alpha_{j_{j_{i}}}^{\left(j_{j_{i}}\right)}+\cdots+\alpha_{r_{j}}^{\left(j_{j}\right)}\left(1-\frac{1}{p_{j_{i}}^{j_{j}}}\right)
$$

(3)

reguläre Untergruppen vom Grade $\delta=\prod_{i=1}^{i=t} p_{j_{i}}^{k_{j}}$, wenn mit $l_{j_{i}}$ die Anzahl derjenigen Zahlen der Reihe $a_{1}^{\left(j_{i}\right)}, \ldots, \alpha_{r_{i}}^{\left(j_{i}\right)}$ bezeichnet wird, welche nicht kleiner als $k_{j_{i}} \operatorname{sind} .\left(k_{j_{i}} \leqq a_{1}^{\left(j_{i}\right)}\right)$

Speciell erhält man z. B. als Anzahl der Untergruppen vom Grade $\prod_{i=1}^{i=t} p_{j_{i}}$, welche von selbst alle regulär sein müssen, das Product $\prod_{i=1}^{i=t} \frac{p_{j_{i}}^{r^{j}} j_{i}-1}{p_{j_{i}}-1}$. Ferner ist klar, dass wenn die Gleichungen

$$
r_{j_{i}}=1 \quad(i=1,2, \ldots, t)
$$


bestehen, nur ein e reguläre Untergruppe vom Grade $\prod^{i=t} p_{j_{i}}^{k_{j}}$ und somit ïberhaupt nur eine Untergruppe von diesem Grade existiert. (Vergl. Satz 4.)

Das Theorem (3) lehrt zugleich die Anzahl der innerhalb der Gruppe $\mathrm{H}$ auftretenden Elemente von einem jeden Grade, welcher Theiler des Grades von $\mathrm{H}$ ist, kennen, eine Anzahl, deren Eigenschaften später noch untersucht werden sollen.

Schlieblich sei noch das folgende Theorem erwähnt:

Eine reguläre Gruppe enthält nur reguläre Untergruppen.

Man gelangt zu demselben ohne Schwierigkeit, wenn man neben dem Satze (1) dieser Nr. den Satz (3) der Nr. 3 in Betracht zieht. Vielleicht entbehrt es aber nicht des Interesses, auch einen Beweis für diesen Satz unabhängig von der im Vorstehenden entwickelten Theorie zu führen.

Die vorgelegte reguläre Gruppe $\Gamma$ sei durch die Reihe

$$
1, B, \ldots, B^{n-1}
$$

gegeben.

Aus einer beliebigen Untergruppe $\Delta$ von $\Gamma$ wähle man ein Element $B^{\alpha}$, dessen Gradzahl den größten Wert $\lambda$ unter den Gradzahlen aller Elemente der betrachteten Untergruppe $\Delta$ besitzt.

Der Exponent $\alpha$ dieses Elementes hat dann die Form $\frac{n}{\lambda} \alpha^{\prime}$, wo unter $\alpha^{\prime}$ eine zu $\lambda$ relativ prime Zahl zu verstehen ist. Man erkennt nun leicht, dass die reguläre Untergruppe

$$
1, B^{\alpha^{\prime} \frac{n}{\lambda}}, \ldots, B^{(\lambda-1) \alpha^{\prime} \frac{n}{\lambda}}
$$

von $\Delta$ nicht nur das Element $B^{\frac{n}{\lambda}}$, sondern auch allgemein ein jedes Element von $\Gamma$, dessen Grad $\lambda^{\prime}$ ein Theiler von $\lambda$ ist, enthält.

In der That besitzt ein solches Element die Form $B^{\frac{n}{\lambda^{\prime}}} a^{\prime \prime}$, wo die beiden Zahlen $\alpha^{\prime \prime}$ und $\lambda^{\prime}$ theilerfremd sind, so dass die in Betracht kommende Congruenz

$$
\alpha^{\prime \prime} \frac{n}{\lambda^{\prime}}=x \alpha^{\prime} \frac{n}{\lambda}(\bmod n)
$$

stets lösbar ist.

Nimmt man nun an, dass die Reihe (I) die Gruppe $\Delta$ noch nicht erschöpft, so muss sich mindestens ein Element $B^{\beta}$ innerhalb 
der letzteren vorfinden, dessen Grad $\mu$ kein Theiler der Zahl $\lambda$ ist. Neben dem Elemente $B^{\beta}$ kommt aber dann auch sicher das Product

$$
B^{y \frac{n}{2}+\beta}
$$

in der Gruppe $\Delta$ vor, wo mit $y$ eine vorläufig noch willkürliche ganze Zahl bezeichnet wird.

Da die Zahl $\beta$ die Form $\beta^{\prime} \frac{n}{u}$ besitzt, in der unter $\beta^{\prime}$ eine zu $\mu$ theilerfremde Zahl zu verstehen ist, kann der Exponent $y \frac{n}{\lambda}+\beta^{\prime} \frac{n}{\mu}$ des obigen Productes auf die Gestalt

$$
\frac{n}{v}\left(y \frac{v}{\lambda}+\beta^{\prime} \frac{v}{\mu}\right)
$$

gebracht werden, falls mit $v$ das kleinste gemeinschaftliche Vielfache von $\lambda$ und $\mu$ bezeichnet wird.

Beachtet man, dass die beiden Zahlen $\frac{v}{\lambda}$ und $\beta^{\prime} \frac{v}{u}$ theilertremd sind, weil der Quotient $\frac{v}{\lambda}$ nur Primfactoren, die in $\mu$, also nicht in $\beta^{\prime}$ aufgehen, enthält, und wählt man für $y$ das Product derjenigen Primzahlen, welche etwa in $\lambda$ und $\mu$ in gleich hoher Potenz enthalten sind, so sieht man, dass der Factor $y \frac{v}{\lambda}+\beta^{\prime} \frac{v}{\mu}$ relativ prim zu $v$ wird. Das Element $B^{y \frac{n}{\lambda}+\beta}$, welches der Gruppe $\Delta$ angehört, besitzt mithin den Grad $v$, der größjer als $\lambda$ ist.

Die Annahme, dass die Reihe (I) noch nicht die Gruppe $\Delta$ erschöpfe, führt mithin auf ein Resultat, das der Definition der Zahl $\lambda$ widerspricht. Das heisst: Die Gruppe $\Delta$ ist identisch mit der Reihe (I), ist also regulär, wie nachzuweisen war.

\section{Nr. 6. Über die Gleichung $\boldsymbol{L} \boldsymbol{X}^{\delta}=\boldsymbol{M}$.}

Da das Product $L X$ mit $X$ zugleich die ganze Gruppe $H$, der das Element $L$ angehört, durchläuft, wird es einmal und nur einmal gleich irgend einem bestimmten Element $M$ von H. D. b.: Jede.Gleichung ersten Grades $L X=M$ besitzt (1) im Gebiete jeder Gruppe, der die Elemente $L$ und $M$ angehören, eine und nur eine Wurzel.

Auf Grund derselben Betrachtungen erhält man die Relation:

$$
\Pi L X=\Pi X,
$$

in der sich die Productzeichen auf alle Elemente $X$ der Gruppe $\mathrm{H}$ vom Grade $n$ zu beziehen haben. Ist diese eine $\mathrm{Abel}$ 'sche, so folgt vermöge des commutativen Gesetztes die Gleichung

$$
L^{n}=1 \text {. }
$$


D. h. :

In jeder Abel'sehen Gruppe vom Grade $n$ hat die Gleichung $X^{n}=1$ alle $n$ Elemente zu Wurzeln.

Dieser Satz gilt übrigens allgemein für jede Gruppe vom Grade $n$, weil der Grad eines jeden Elementes derselben Theiler von $n$ sein muss.

Man schließt daraus, dass ein System von $n$ Zahlen im gewöhnlichen complexen Gebiet dann und nur dann hinsichtlich der Multiplication Gruppeneigenschaft besitzt, wenn es abgesehen von der Einheit mit der Gesammtheit der $n^{\text {ten }}$ Einheitswurzeln übereinstimmt. Bekanntlich lässt sich eine solche Gruppe durch die Potenzen einer primitiven $n^{\text {ten }}$ Einheitswurzel darstellen; sie ist also regulär.

Es wurde bereits in der Nr. 4 darauf hingewiesen, dass die Gesammtheit der Wurzeln der Gleichung:

$$
X^{\delta}=1
$$

im Gebiete der Abel'schen Gruppe $\mathrm{H}$ selbst eine Untergruppe $\mathrm{H}_{\delta}$ von $\mathrm{H}$ bildet.

Der Grad von $H_{\delta}$ ist natürlich stets Theiler von $n$, jedoch nach dem Satze (2) dann und nur dann ein Multiplum von $\delta$, wenn die Zahl $n$ durch $\delta$ theilbar ist; denn in diesem Falle existiert nach den Ausführungen der vorigen Nr. sicher eine Untergruppe von $\mathrm{H}$ vom Grade $\delta$.

$\mathrm{Um}$ aber den Grad von $\mathrm{H}_{\delta}$ wirklich zu bestimmen, setzen wir hier, wie überhaupt im Folgenden, voraus, die Gruppe $\mathrm{H}$ sei durch die Basiselemente $B_{i}$ mit den Gradzahlen $n_{i}(i=1,2, \ldots, s)$, die beliebig zusammengesetzte Zahlen sein können, gegeben.

Dann folgt aus der Gleichung

$$
\left(\prod_{i=1}^{i=s} B_{i}^{\alpha_{i}}\right)^{\delta}=1
$$

auf Grund der Eindeutigkeit der Darstellung eines jeden Elementes von $\mathbb{H}$ durch die Basis $B_{i}$ die Beziehung

$$
\alpha_{i} \delta=\mu_{i} n_{i}
$$

für $i=1,2, \ldots, s$, in welcher $\mu_{i}$ eine ganze Zahl bedeutet. Bezeichnet man daher mit $\delta_{i}$ den gröbten gemeinsamen Theiler zwischen $\delta$ und $n_{i}$, so muss der Exponent $\alpha_{i}$ ein Multiplum von $\frac{n_{i}}{\delta_{i}}$ sein. 
Umgekehrt wenn die Zahl $\alpha_{i}$ ein Multiplum von $\frac{n_{i}}{\delta_{i}}(i=1, \ldots, s)$ ist, so stellt das Element $\prod_{i=1} B_{i}^{\alpha_{i}}$ eine Wurzel der Gleichung: $X^{\delta}=1$ vor.

Die Gruppe $\mathrm{H}_{\delta}$ erscheint somit durch die Gesammtheit der Elemente

$$
\prod_{i=1}^{i=s} B_{i}^{\frac{n_{i}}{\delta_{i}} \mu_{i}} \quad\left(\begin{array}{c}
\mu_{i}=0,1, \ldots, \overline{\delta_{i}-1} \\
i=1, \ldots, s
\end{array}\right)
$$

dargestellt, woraus sich die beiden folgenden Theoreme ergeben: Die Zahl der Wurzeln der Gleichung $X^{\delta}=1$ im Gebiete einer Abel'schen Gruppe, deren Basiselemente die Gradzahlen $n_{i}(i=1,2, \ldots, s)$ besitzen, $i=s$

(3) wird durch das Product $\prod_{i=1} \delta_{i}$ bestimmt, wenn unter $\delta_{i}$ der grölote gemeinsame Theiler von $\delta$ und $n$ verstanden wird.

Die Gruppen $H_{\delta}$ und $H_{\delta^{\prime}}$ sind identisch, wenn (4) die Zahlen $\delta$ und $\delta^{\prime}$ denselben gröhten gemeinsamen Theiler mit $n$ besitzen.

Die zwei folgenden speciellen Fälle, bei denen die Zahl $\delta$ als Divisor von $n$ angenommen wird, mögen herrorgehoben werden:

Ist erstens der Quotient $\frac{n}{\delta}$ zu $\delta$ relativ prim, so besitzt die Gruppe $\mathrm{H}_{\delta}$ den Grad $\delta$, wie dies auch aus den Betrachtungen der Nr. 4 folgt.

Sind zweitens je zwei der Zahlen $n_{1}, \ldots, n_{s}$ mit einander theilertremd, ist also die Gruppe $\mathrm{H}$ regulär, so besitzt die Untergruppe $\mathrm{H}_{\delta}$ ebenfalls den Grad $\delta$.

Es ist klar, dass jedes Element, das simultan die beiden Gleichungen

$$
X^{\delta}=1 \text { und } X^{\delta^{\prime}}=1
$$

befriedigt, einen Grad besitzt, der gleichzeitig $\delta$ und $\hat{o}^{\prime}$ und damit auch den gröliten gemeinsamen Divisor $=$ von $\delta$ und $\delta^{\prime}$ theilt. Ein jedes solche Element ist also auch Wurzel der Gleichung:

$$
X^{\tau}=1 \text {. }
$$

Da ebenso die Umkehrung hiervon gilt, folgt der Satz:

Der grölte gemeinsame Divisor derbeiden (5) Gruppen $H_{\delta}$ und $H_{\delta^{\prime}}$ wird $d \mathbf{u r c h}$ die Untergruppe $\mathrm{H}_{\tau}$ gebildet, wo $\tau$ den größten gemeinsamen Theiler der beiden Zahlen $\delta$ und $\delta^{\prime}$ vorstellt. 
Aus diesem Satze erkennt man wieder die Giltigkeit des Satzes (4), wenn man den größten gemeinsamen Divisor einerseits zwischen $\mathrm{H}$ und $\mathrm{H}_{\delta}$, andererseits zwischen $\mathrm{H}$ und $\mathrm{H}_{\delta^{\prime}}$ bildet ${ }^{1}$ ).

Schließlich sei bemerkt, dass das Product aller Elemente von $\mathrm{H}_{\delta}$ leicht auf Grund der Form (I) derselben gebildet werden kann. Man erhält als Resultat das Element

$$
\prod_{i=1}^{i=s} B_{i}^{\frac{n_{i}\left(\delta_{i}-1\right)}{2}} \delta_{1} \ldots \delta_{i-1} \delta_{i+1} \ldots \delta_{s},
$$

welches sich anf die Einheit reduciert, vorausgesetzt, dass man nicht alle Zahlen $\delta_{1}, \ldots, \delta_{i-1}, \hat{o}_{i+1}, \ldots, \delta_{s}$ ungerade, die Zahl $\delta_{i}$ aber gerade annimmt. In diesem Falle ergibt sich für das Product aller Elemente von $\mathrm{H}_{\delta}$ das Individuum $B_{i}^{\frac{n_{i}}{2}}$. Satz:

Hieraus folgt, indem man $\delta=n$ setzt, ohne Weiteres der

Besitzt das Element $B_{i}$ allein unter allen Basiselementen der Gruppe $H$ einen geraden (6) Grad, so ist das Product aller Individuen von $\mathrm{H}$ gleich $B_{i}^{\frac{n_{i}}{2}}$. In allen andern Fällen erhält man für dasselbe die Einbeit.

Was die allgemeine Gleichung

$$
L X^{\delta}=M
$$

betrifft, genügt es nach dem Satze (2) jene Fälle zu betrachten, in denen der Exponent $\delta$ die Zahl $n$ nicht überschreitet.

Es ist klar, dass die Gleichung (II) dann und nur dann wenigstens eine Wurzel and damit nach den Ausführungen der Nr. 4 auf Grund des Satzes (3) genau $\prod_{i=1} \delta_{i}$ Wurzeln besitzen wird, wenn das Product $L^{-1} M$ eine $\delta^{\text {te }}$ Potenz vorstellt.

Die Gesammtheit der $\delta^{\text {ten }}$ Potenzen innerhalb der Gruppe $\mathrm{H}$ erscheint ihrer Definition gemäß durch das System der Elemente

(III) $\quad \prod_{i=1}^{i=s} B_{i}^{\delta \alpha_{i}}$ oder $\prod_{i=1}^{i=s} B_{i}^{\delta_{i} \alpha_{i}} \quad\left(\alpha_{i}=0,1, \ldots,\left(\frac{n_{i}}{\delta_{i}}-1\right)\right)$

1) Bezüglich der Ausdehnung dieses Satzes auf mehrere Systeme vergleiche man die Bemerkungen zum Satze (9), sowie auch die nächste Nr. 
dargestellt, indem ja die Reihe $0, \hat{\delta}, \ldots,\left(\frac{n_{i}}{\delta_{i}}-1\right) \delta$ modulo $n_{i}$ vollständig mit der Reihe $0, \delta_{i}, \ldots,\left(\frac{n_{i}}{\delta_{i}}-1\right) \delta_{i}$ übereinstimmt.

Aus dieser Darstellung ergeben sich ohne Weiteres die drei folgenden Sätze (7), (8) und (9).

Die $\delta^{\text {ten }}$ Potenzen innerhalb der Gruppe $\mathrm{H}$ bilden selbst eine Gruppe $\Gamma_{\delta}$ rom Grade

$$
\prod_{i=1}^{\frac{n}{i=s} \delta_{i}} .
$$

Es folgt dieser Satz naturlich auch aus dem Satze (3) auf Grund des Schemas (î) der Nr. 4.

Wenn die beiden Zahlen $\delta$ und $\sigma^{\prime}$ mit $n$ den-

selben gröbten gemeinsamen Divisor besitzen, so sind die Gruppen der $\delta^{\text {ten }}$ und $\delta^{\text {ten }}$ Potenzen miteinander identisch.

Mithin existieren nur soviele verschiedene Systeme von $\delta^{\text {ten }}$ Potenzen innerhalb der Grappe H, als es Theiler ò von $n$ gibt, je $:\left(\frac{n}{\delta}\right)$ Systeme fallen zusammen.

Den größ $\beta$ ten gemeinsamen Theiler der beiden (9)

Gruppen $\Gamma_{\delta}$ und $\Gamma_{\delta^{\prime}}$ stellt die Untergruppe $\Gamma_{\frac{\delta \delta^{\prime}}{\tau}}$ dar,

wenn unter c der gröbte gemeinsame Divisor der beiden Zahlen $\delta$ und $\delta^{\prime}$ verstanden wird.

Dieser Satz lässt sich übrigens verallgemeinern: Versteht man unter $v\left(\bar{\delta}_{1}, \ldots, \bar{\delta}_{r}\right)$ das kleinste gemeinsame Vielfache der Zahlen $\bar{\delta}_{1}, \ldots, \hat{o}_{r}$, so bildet das System der $v\left(\bar{\delta}_{1}, \ldots, \bar{\delta}_{r}\right)^{\text {ten }}$ Potenzen die größte gemeinsame Untergruppe der $\overline{\hat{o}}_{1}^{\text {ten }},{\overline{\delta_{2}}}_{2}^{\text {ten }}, \ldots, \bar{\delta}_{r}^{\text {ten }}$ Potenzen.

Damit wird zugleich die Frage nach dem Complex derjenigen Elemente von $\mathrm{H}$, welche weder $\bar{\delta}_{1}^{\text {te }}$, noch $\bar{\delta}_{2}^{\text {te }}, \ldots$, noch $\overline{\hat{\delta}}_{r}^{\text {te }} \mathrm{Po}_{0}$ tenzen sind, erledigt.

Gemälb dem Ausdrucke (I) der Nr. 1 im I. Abschn. stellt sich derselbe in der Form

$$
\mathrm{H}-\dot{\mathbf{Z}}_{\bar{\delta}} \Gamma_{\bar{\delta}} \dot{+} \sum_{\bar{\delta}, \overline{\delta^{\prime}}} \Gamma_{v\left(\bar{\delta}, \overline{\delta^{\prime}}\right)} \dot{-} \cdots
$$

dar. Daraus ergibt sich z. B., dass die Anzahl derjenigen Elemente einer regulären Gruppe vom Grade $n$, welehe weder 
$\bar{\delta}_{1}^{\text {te }}$, noch $\bar{\delta}_{2}^{\text {te }}, \ldots$, noch $\bar{\delta}_{r}^{\text {te }}$ Potenzen sind, gleich dem Producte $n \prod_{i=1}^{i=r}\left(1-\frac{1}{\delta_{i}}\right)$ ist, wenn je zwei der Zahlen $\bar{\delta}_{1}, \ldots, \bar{\delta}_{r}$ theilerfremd. sind und überdies $n$ ein Multiplum des Productes $\bar{\delta}_{1} \bar{\delta}_{2} \ldots \bar{\delta}_{r}$ vorstellt.

Für das Product aller $\delta^{\text {ten }}$ Potenzen erhält man das Element

$$
\prod_{i=1}^{i=s} B_{i}^{\frac{n_{i}}{2}}\left(\frac{n_{i}}{\delta_{i}}-1\right) \frac{n_{1}}{\delta_{1}} \frac{n_{2}}{\delta_{2}} \cdots \frac{n_{i-1}}{\delta_{i-1}} \frac{n_{i+1}}{\delta_{i+1}} \cdots \frac{n_{s}}{\delta_{s}},
$$

welches sich auf die Einheit reduciert, vorausgesetzt, dass man nicht alle Zahlen $\frac{n_{1}}{\delta_{1}}, \ldots, \frac{n_{i-1}}{\delta_{i-1}}, \frac{n_{i+1}}{\delta_{i+1}}, \ldots, \frac{n_{s}}{\delta_{s}}$ ungerade, die Zahl $\frac{n_{i}}{\delta_{i}}$ aber gerade annimmt. In diesem Falle ergibt sich nämlich für das. Product das Individuum $B_{i}^{\frac{n_{i}}{2}}$.

Wie man sieht, besitzt die Gruppengattung $\Gamma_{\delta}$ ganz analoge Eigenschaften, wie die Gruppengattung $\mathrm{H}_{\delta}{ }^{1}$ ); es ist daher interessant, auf ihr gegenseitiges Verhältnis näher einzugehen.

Die Form (III), welche alle $\delta^{\text {ten }}$ Potenzen von $\mathrm{H}$ charakterisiert, lehrt, dass jeder Exponent, auf den alle $\delta^{\text {ten }}$ Potenzen erhoben werden müssen, um ausnahmslos gleich der Einheit zu werden, nothwendig ein Multiplum des kleinsten gemeinsamen Vielfachen $v$ der Zahlen $\frac{n_{1}}{\delta_{1}}, \cdots, \frac{n_{s}}{\delta_{s}}$ sein muss; umgekehrt leuchtet ein, dass die Zahl $v$ thatsächlich die angeführte Forderung erfüllt. Die Gleichung

$$
X^{v}=1
$$

besitzt also alle $\delta^{\text {ten }}$ Potenzen zu Wurzeln und es existiert keine analoge Gleichung niedrigeren Grades von derselben Eigenschaft.

Die Gruppe $\Gamma_{\delta}$ ist mithin Untergruppe vom $\mathrm{H}_{v}$.

Keineswegs gilt aber im Allgemeinen das Umgekehrte; denn die Elemente von $\mathrm{H}_{v}$ stimmen gemäß der Form (I) genau mit. den Individuen

$$
\prod_{i=1}^{i=s} B_{i}^{{\frac{n_{i}}{\tau_{i}} \mu_{i}}_{i}} \quad\left(\begin{array}{c}
\mu_{i}=0,1, \ldots, \overline{\tau_{i}-1} \\
i=1,2, \ldots, s
\end{array}\right)
$$

1) Diese Analogie geht noch weiter, indem sich innerhalb der Gruppe $\Gamma_{\delta}$ Elemente auffinden lassen, welche vollständig den der Gruppe $\mathrm{H}_{\delta}$ angehörigen Individuen vom Grade $\delta$ entsprechen. Man vergleiche diesbezüglich den $\S .3 \mathrm{der}$ Nr. 3 des nächsten Abschnittes. 
überein, wo unter $\tau_{i}$ der größte gemeinsame Divisor zwischen $n_{\imath}$ und $v$, also ein Multiplum von $\frac{n_{i}}{\dot{\delta}_{i}}$ zu verstehen ist, und werden somit insgesammt dann und nur dann entsprechend der Form (III) gleichzeitig $\delta^{\text {te }}$ Potenzen sein, wenn sich zu jedem Wertsystem $\mu_{1}, \ldots, \mu_{s}\left(\mu_{i}=0,1, \ldots, \overline{\tau_{i}-1}\right)$ Zahlen $x_{1}, \ldots, x_{s}$ der Art finden lassen, dass die Congruenzen

$$
x_{i} \delta_{i} \equiv \frac{n_{i}}{\tau_{i}} \mu_{i}\left(\bmod n_{i}\right) \text { für } i=1,2, \ldots, s
$$

erfüllt sind.

Diese Congruenzen haben aber stets und nur dann Wurzeln, wenn die $s$ Bedingungen

$$
\tau_{i}=\frac{n_{i}}{\delta_{i}} \quad(i=1,2, \ldots, s)
$$

zu Recht bestehen, d. h. wenn kein Primfactor von $\delta_{i}$ in höherer Potenz in irgend einer der Zahlen

$$
\frac{n_{j}}{\delta_{j}}(j=1,2, \ldots, \overline{i-1}, \overline{i+1} \ldots s),
$$

wie in $\frac{n_{i}}{\delta_{i}}$ enthalten ist für $i=1,2, \ldots, s$.

Man ist mithin zu dem folgenden Theorem gelangt:

Das System der $\delta^{\text {ten }}$ Potenzen erschöpf́t dann und nurdann die Gesammtheit der Wurzeln der Gleichung $X^{v}=1$, wenn jeder Primfactor von $\delta$, (10) der wenigstens in $z$ wei der Zahlen $n_{1}, \ldots, n_{s}$ in verschiedener Potenz aufgeht, die Zahl $\delta$ in einer Potenz theilt, welche nicht kleiner ist als die höchste Potenz, mit der er in die Zahlen $n_{1}, \ldots, n_{s}$ eingeht.

Natürlich ist in allen den Fällen, in denen die Bedingungen dieses Satzes erfüllt sind, ein Kriterium für die $\delta^{\text {ten }}$ Potenzen gewonnen:

Das Element $A$ ist dann und nur dann $\delta^{\text {te }}$ Potenz, wenn die Gleichung $A^{v}=1$ besteht und je nachdem diese Beziehung stattfindet oder nicht, hat die Gleichung $X^{\delta}=A$ genau $\prod_{i=1} \delta_{i}$ Wurzeln oder überhaupt keine. 
Unter den speciellen Fällen des Theoremes (10) mögen die beiden folgenden besonders hervorgehoben werden:

Sind erstens die beiden ganzen Zahlen $\frac{n}{\delta}$ and $\delta$ theilerfremd, so stimmen die $\delta^{\text {ten }}$ Potenzen mit der Gruppe $\mathrm{H}_{n}$ überein. In diesem Falle sind nämlich die beiden Gruppen $\mathrm{H}_{v}$ nnd $\mathrm{H}_{\frac{n}{\delta}}$ identisch, wie sich leicht aus der Form (I) schließen lässt.

Nimmt man zweitens je zwei der Gradzahlen $n_{1}, \ldots, n_{s}$ mit einander theilerfremd an, so erhält mau den folgenden Satz:

Innerhalb einer regulären Gruppe vom Grade $n$ stimmen die $\delta^{\text {ten }}$ Potenzen mit den verschiedenen

Wurzeln der Gleichung $X^{\frac{n}{t}}=1$ überein, wo unter $\tau$ der gröbte gemeinsame Theiler $z$ wischen $n$ und $\delta \mathrm{zu}$ verstehen ist.

Wie man sieht, gestalten sich die Verhältnisse im Falle, als eine reguläre Gruppe $\mathrm{H}$

$$
1, B, B^{2}, \ldots, B^{n-1}
$$

vorliegt, sehr einfach: Für die Untergruppe $\mathrm{H}_{n}$ kann die Gruppe $\Gamma_{\delta}$, d. h. die Reihe $1, B^{\delta}, \ldots, B^{\left(\frac{n}{\tau}-1\right) \delta}$ gesetzt werden, so dass das Schema (î) der Nr. 4 die Form

$\left(\mathfrak{A}_{\delta}\right)\left\{\begin{array}{l}(0) \quad 1, B^{\delta}, B^{2 \delta}, \ldots, B^{\left(\frac{n}{\tau}-1\right) \delta} \\ (1) \quad B, B^{\delta+1}, B^{2 \delta+1}, \ldots, B^{\left(\frac{n}{\tau}-1\right) \delta+1} \\ \cdot \cdot \cdot \cdot \cdot \cdot \cdot \cdot \cdot \cdot \cdot \cdot \cdot B^{\left(\frac{n}{\tau}-1\right) \delta+\overline{\tau-1}}\end{array}\right.$

annimmt, sobald man in demselben für den Index $\delta$ die Zahl $\frac{n}{\tau}$ und für das Element $S_{i}$ die Potenz $B^{i}(i=1,2, \ldots, \overline{\tau-1})$ substituiert.

Das Schema $\left(\mathfrak{A}_{\delta}\right)$ spielt bei manchen Untersuchungen eine Rolle und es mag daher eine Zusammenstellung einiger Eigenschaften desselben hier Platz finden.

Wie man sieht, zerfallt die Gruppe $H$ in $\tau$ Klassen mit je $\frac{n}{\tau}$ Elementen der Art, dass die Klasse $(0)$ mit allen $\delta^{\text {ten }}$ Potenzen 
von $\mathrm{H}$ und überhaupt die Klasse $(i)(i=0,1, \ldots, \overline{z-1})$ mit der Gesammtheit derjenigen Elemente von $\mathrm{H}$ identisch ist, welche auf die $\left(\frac{n}{\tau}\right)^{\text {te }}$ Potenz erhoben ein und dasselbe Element $B^{i \frac{n n}{\tau}}$ ergeben. Die Gleichung $X^{\frac{n}{\tau}}=B^{i \frac{n}{\tau}}$ ist also für alle Elemente der $i^{\text {ten }}$ Klasse charakteristisch. 1 )

Es erweist sich übrigens als vortheilhaft, die Anzahl der Klassenzeiger zu erweitern; naturgemäß wird unter einer Klasse mit einem die Zahl $\overline{\tau-1}$ übersteigenden Zeiger $k$ diejenige Klasse des Schemas $\left(\mathfrak{H}_{\delta}\right)$ zu verstehen sein, deren Zeiger mit $k$ modulo $\tau$ congruent ist.

Gehört dann das Element $A$ der $i^{\text {ten, }}$, das Element $A^{\prime}$ jedoch der $i^{\text {ten }}$ Klasse an, so wird sich nach dieser Festsetzung das Product $A A^{\prime}$ in der $\left(i+i^{\prime}\right)^{\text {ten }}$ Klasse vorfinden müssen, und man erkennt, dass das Resultat der Multiplication eines jeden Elementes einer Klasse mit irgend einem Elemente einer $2^{\text {ten }}$ Klasse stets nur ein Individuum einer und derselben $3^{\text {ten }}$ Klasse sein kann.

1) Die Elemente

$$
1, B^{\frac{n}{\tau}}, \ldots, B^{(\tau-1) \frac{n}{\tau}}
$$

bilden natïrlich die Gesammtheit der $\left(\frac{n}{\tau}\right)^{\text {ten }}$ Potenzen der Gruppe $H$, und zwar gehören je $\theta$ von ihnen den Klassen

$$
(0) ;(\Theta) ; \ldots ;\left(\left(\frac{\tau}{\theta}-1\right) \theta\right)
$$

des Schemas $\left(\mathfrak{H}_{\delta}\right)$ an, falls' unter $\theta$ der größte gemeinsame Divisor zwischen $\tau$ und $\frac{n}{\tau}$ verstanden wird.

Es folgt dies daraus, dass die Congruenz

$$
j-f-x \tau \equiv y \frac{n}{\tau}(\bmod n)
$$

zunächst die Beziehung

$$
j \equiv 0(\bmod \theta) \text { und hierauf } \frac{j}{\theta}+x \frac{\tau}{\theta} \equiv 0\left(\bmod \frac{n}{\tau \theta}\right)
$$

nach sich zieht. Während die erstere dieser beiden Congruenzen die Klassen angibt, welche überhaupt $\left(\frac{n}{\tau}\right)^{\text {te }}$ Potenzen enthalten können, vermittelt die letztere die Anzahl der in einer solchen Klasse enthaltenen $\left(\frac{n}{\tau}\right)^{\text {ten }}$ Potenzen.

Bezeichnet man nämlich mit $\alpha$ eine bestimmte Wurzel der letzteren Congruenz, so hat eine jede andere Wurzel $x$ die Form $\alpha+x^{\prime} \frac{n}{\tau \theta}\left(x^{\prime}= \pm 1, \pm 2, \ldots\right)$. Es existieren also für das Product $x \tau$ höchstens $\Theta$ modulo $n$ verschiedene Zahlen, die der aufgestellten Forderung möglicher Weise Genüge leisten. Thâtsächlich findet sich nun zu jedem solchen Wert $x$ eine entsprechende Lösung $y$ der ursprünglichen Congruenz, so dass sich in jeder möglichen Klasse $(j)$ genau $\theta$ Elemente der bezeichneten Art vorfinden. 
Es sei bemerkt, dass einige der oben für die Gruppe der $\delta^{\text {ten }}$ Potenzen hervorgehobenen Sätze allgemein für die $i^{\text {te }}$ Klasse des Schemas $\left(\mathfrak{U}_{\delta}\right)$ gelten.

So ist es klar, dass, wenn die beiden Zahlen $\delta$ und $\delta^{\prime}$ mit $n$ denselben grölten gemeinsamen Theiler $\tau$ besitzen, auch das Schema $\left(\mathfrak{H}_{\delta^{\prime}}\right)$ abgesehen von der Aufeinanderfolge der Elemente die nämlichen Klassen, wie das Schema $\left(\mathfrak{H}_{\delta}\right.$ ) umfasst. (Vergl. Satz (8).)

Ebenso besteht der folgende Satz:

Ist die Zahl o das kleinstegemeinsame Vielfache der beiden Zahlen $\delta_{1}$ und $\grave{o}_{2}$, sostimmen die (12) Elemente der Klasse (i) im Schema $\left(\mathfrak{U}_{\delta}\right)$ mit der Gesammtheit der gemeinsamen Elemente der Klassen $(i)$ in $\left(\mathfrak{A}_{\delta_{1}}\right)$ and $\left(\mathfrak{H}_{\delta_{2}}\right)$ überein. (Vgl. Satz (9).) Gehört nämlich das Element $S=B^{k}$ im Schema $\left(\mathfrak{A}_{\delta_{1}}\right.$ ) derselben Klasse (i), wie im Schema $\left(\mathfrak{A}_{\delta_{2}}\right)$ an, so bestehen die beiden Gleichungen:

$$
B^{k}=B^{i+\delta_{1} \mu_{1}} \text { und } B^{k}=B^{i+\delta_{2} \mu_{2}}
$$

aus denen sich die beiden Congruenzen

$$
k-i \equiv \mu_{1} \dot{\delta}_{1} \text { und } k-i \equiv \mu_{2} \delta_{2} \quad(\bmod n)
$$

ergeben. Versteht man also unter $\tau_{1}$, bezw. $\tau_{2}$ das größte gemeinsame Maß3 zwischen $\delta_{1}$, bezw. $\delta_{2}$ und $n$, so muss die Differenz $k-i$ durch $\tau_{1}$ und $\tau_{2}$, mithin auch durch das kleinste gemeinsame Vielfache $\tau$ dieser beiden Zahlen theilbar sein. Da aber, wie eine einfache Überlegung lehrt, ${ }^{1}$ ) die Zahl $\tau$ der größte gemeinsame Theiler zwischen $n$ und dem kleinsten gemeinsamen Vielfachen $\delta$ von $\hat{\delta}_{1}$ und $\delta_{2}$ ist, hat die Congruenz

$$
k-i \equiv x \grave{o}(\bmod n)
$$

1) Es bestehen nämlich unter den gemachten Annahmen die Gleichungen:

$$
\begin{array}{ll}
\delta_{1}=\delta_{1}^{\prime} \tau_{1} ; & n=n_{1} \tau_{1} \\
\delta_{2}=\delta_{2}^{\prime} \tau_{2} ; & n=n_{2} \tau_{2},
\end{array}
$$

wobei $\delta_{1}^{\prime}$ mit $n_{1}$ und $\delta_{2}^{\prime}$ mit $n_{2}$ theilerfremd ist. Ferner leuchtet von selbst ein, dass die beiden Zahlen $\delta$ und $n$ Multipla von $\tau$ sind, so dass man

setzen kann.

$$
\delta=\bar{\delta} \tau \text { and } n=\bar{n} \tau
$$

Aus diesen Gleichungen folgt unmittelbar, dass jeder Primzahlfactor $p$ von $\bar{n}$, auch Primfactor von $n_{1}$ und $n_{2}$ sein muss, also weder in $\delta_{1}^{\prime}$ noch in $\delta_{2}^{\prime}$ aufgehen darf, das heißt, er ist in $\delta_{1}$, bezw, in $\delta_{2}$ in der nämlichen Potenz enthalten, wie in $\tau_{1}$, bezw. $\tau_{2}$. Das Gleiche gilt dann aber anch beziuglich des kleinsten gemeinsamen Vielfachen $\delta$ ron $\delta_{1}$ und $\delta_{2}$ und desjenigen der beiden Zahlen $\tau_{1}$ und $\tau_{2}$, so dass der Quotient $\frac{\delta}{\tau}$ überhaupt den Primfactor $p$ nicht besitzt. 
eine Wurzel; das Element $S$ gehört demnach der Klasse (i) im Schema $\left(\mathfrak{U}_{\delta}\right)$ an.

Findet sich umgekehrt das Element $S$ in der Klasse (i) des Schemas $\left(\mathfrak{A}_{\delta}\right)$ vor, so gilt natürlich dasselbe für $\left(\mathfrak{H}_{\delta_{1}}\right)$ und $\left(\mathfrak{H}_{\delta_{2}}\right)$. Überhaupt leuchtet ein, dass die Klassen $(i),\left(i+\tau_{1}\right), \ldots$, $\left(i+\left(\frac{\tau}{\tau_{1}}-1\right) \tau_{1}\right)$ des Schemas $\left(\mathfrak{A}_{\delta}\right)$ zusammengenommen genau die $i^{\text {te }}$ Klasse von $\left(\mathfrak{A}_{\delta_{1}}\right)$ constituieren.

Aus dem Theoreme (12) folgt speciell der Satz:

Ist die Zahl $\delta$ in ihre Primfactoren zerlegt gleich dem Producte $q_{1}^{k_{1}} \ldots q_{r}^{k_{r}}$, so gehört das Element $S$ dann und nur dann der $i^{\text {ten }}$ Klasse des Schemas $\left(\mathfrak{A}_{\delta}\right)$ an, wenn dasselbe auch in jedem Schema $\left(\mathfrak{A}_{q_{j} k^{k}}\right)(j=1,2, \ldots, r)$ in der Klasse $(i)$ enthalten ist.

Schlieblich soll noch untersucht werden, inwieferne das Schema $\left(\mathfrak{H}_{\delta}\right)$ von der Wahl des Basiselementes abhängt; es können ja an Stelle von $B$ genau $\varphi(n)$ der Gruppe $H$ angehörige Elemente genommen werden.

Setzt man für $B$ im Schema $\left(\mathfrak{A}_{\delta}\right)$ das Element $B^{\circ}$, oder erhebt man, was dasselbe ist, alle Individuen von $\left(\mathfrak{H}_{\delta}\right)$ auf die $p^{\text {te }}$ Potenz, wobei $\rho$ theilerfremd mit $n$ vorausgesetzt wird, so erkennt man zunächst, dass die Klassen als solche, abgesehen von der Reihenfolge ihrer Elemente ungeändert bleiben. Denn die Reihe

$$
0, \delta, \ldots,\left(\frac{n}{\tau}-1\right) \delta
$$

welche alle modulo $n$ verschiedenen Zahlen angibt, die mit $n$ den Theiler $\tau$ gemeinsam haben, kann modulo $n$ abgesehen von der Aufeinanderfolge der Zahlen auch in der Form

$$
0, \text { pò, ..., }\left(\frac{n}{\tau}-1\right) \text { pò }
$$

geschrieben werden.

Was hingegen die Reihenfolge der Klassen selbst betrifft, so wird die Ordnungszahl $j$ der trüheren $i^{\text {ten }}$ Klasse des Systemes $\left(\mathfrak{A}_{\delta}\right)$ in der neuen Anordnung durch die Congruenz

bestimmt.

$$
j \equiv i_{p} \quad(\bmod \tau)
$$

Es folgt hieraus zunächst der Satz, dass alle Klassen, deren Ordnungszahl (i) denselben größten gemeinsamen Theiler $\Theta$ mit der Zahl $\tau$ besitzen, sich durch die Neuanordnung nur untereinander vertauschen; gehört also die Zahl $\rho$ zum Exponenten $k$ modulo $\frac{\tau}{\bar{\theta}}$, so vertauschen sich je $k$ der $\varphi\left(\frac{\tau}{\tilde{\theta}}\right)$ Klassen eines jeden solchen Systemes. 
Vergleicht man ferner zwei Nenanordnungen des Systemes $\left(\mathfrak{A}_{\delta}\right)$, welche zu den Zahlen $\rho$ und $\rho^{\prime}$ gehören, so liefert die erwähnte Congruenz ohne Weiteres den Satz, dass die Wahl zweier Basiselemente $B^{\varrho}$ und $B^{\varrho^{*}}$, deren Exponenten modulo $\tau$ congruent sind, identische Klassenanordnungen ergibt.

Dieser Satz lehrt zunächst, dass es sicher nicht mehr als $\tau$ in der Klassenanordnung unterschiedene Schemata $\left(\mathfrak{A}_{\delta}\right)$ geben kann. Da jedoch die Zahlen $p$ relativ prim zu $n$, also auch relativ prim zu dem Theiler $\tau$ von $n$ sein müssen, gibt es sogar nicht mebr als $p(\tau)$ verschiedene Systeme $\left(\mathfrak{U}_{\delta}\right)$. Es kann aber anch deren nicht weniger geben, indem stets Zahlen $p$ existieren, welche modulo $\tau$ mit einer gegebenen zu $\tau$ theilerfremden Zahl congruent sind. Die Verhältnisse gestalten sich am einfachsten, wenn in den Zahlen $n$ und $\tau$ dieselben Primzahlen enthalten sind, so dass jede $\mathrm{zu} \tau$ relativ prime Zahl auch $\mathrm{zu} n$ relativ prim sein muss. In diesem Falle ordnen sich alle möglichen $p(n)$ Basiselemente $B^{o}$ den $\varphi(\tau)$ verschiedenen Systemen $\left(\mathfrak{H}_{\delta}\right)$ der $\operatorname{Art} z u$, dass zu jedem speciellen Systeme $\frac{\varphi(n)}{\varphi(\tau)}$ Basiselemente, welche dieselbe Klassenanordnung ergeben, gehören.

Im Falle, dass die Zahl $\div$ gerade ist, erscheint die Klasse $\left(\frac{\tau}{2}\right)$ dadurch ausgezeichnet, dass sie, wie die Klasse (0) unabhängig von der Wahl des Basiselementes ist.

Nr. 7. Über die Anzahl der Elemente eines bestimmten Grades innerhalb einer Abel'schen Gruppe.

Es sei die gegebene Zahl ò in ihre Primfactoren zerlegt gleich dem Producte II $q_{i}^{k_{i}}$; dann kann man die Elemente vom Grade o als diejenigen Wurzeln der Gleichung

$$
X^{\delta}=1
$$

im Gebiete $H$ auffassen, welche nicht zugleich Wurzeln irgend einer der Gleichungen

$$
X^{\frac{\delta}{q_{j}}}=1 \quad(j=1,2, \ldots, r)
$$

sind; denn der Grad einer solchen Wurzel ist wohl Theiler von $\delta$, aber nicht von $\frac{\delta}{q_{j}}(j=1, \ldots, r)$, mithin gleich $\delta$. 
Um den Complex aller Elemente rom Grade ô za finden, hat man mithin nur aus der Gruppe $\mathrm{H}_{\delta}$ alle Untergruppen $\mathrm{H}_{\delta}$ $(j=1, \ldots, r)$ auszuscheiden, was mit Hilfe der Methode des Ausscheidens und Hinzufügens leicht bewerkstelligt werden kann.

Berücksichtigt man das Theorem (5) der vorigen Nr., so ergibt sich der gesuchte Complex nach dem Ausdrucke (I) der Nr. 1 im I. Abschn. in der Form

$$
\mathrm{H}_{\delta} \div \sum_{j} \mathrm{H}_{\frac{\delta}{q_{j}}}+\sum_{j, j^{\prime}} \mathrm{H}_{\frac{\delta}{q_{j} q_{j^{\prime}}}} \cdot \cdots
$$

Nun wird gemä6 dem Theoreme (3) der vorigen Nummer der Grad der Gruppe $\mathrm{H}$ \& durch das Product

$$
\begin{aligned}
& \frac{\delta}{q_{i_{1}} \cdots q_{i_{m}}} \\
& \prod_{j=1}^{j=s}\left(\frac{\delta}{q_{i_{1}} \cdots q_{i_{m}}}\right)_{j}
\end{aligned}
$$

bestimmt, wenn der größfte gemeinsame Divisor zwischen $\frac{\delta}{q_{i_{1}} \cdots q_{i_{g_{b}}}}$ und der Gradzahl $n_{j}$ des Basiselementes $B_{j}$ mit dem Symbol $\left(\frac{\delta}{q_{i_{1}} \cdots q_{i_{m}}}\right)_{j}$ bezeichnet wird. Man erbält also auf Grund des eben gefundenen Ausdruekes (I) für die Anzahl $\bar{p}\left(\delta ; n_{1}, \ldots, n_{s}\right)$ der Elemente vom Grade $\delta$ innerhalb der Gruppe $H$ die Gleichung

(II) $\bar{\varphi}\left(\delta ; n_{1}, \ldots, n_{s}\right)=\prod_{j=1}^{j=s} \delta_{j}-\sum_{i} \prod_{j=1}^{j=s}\left(\frac{\delta}{q_{i}}\right)_{j}+\sum_{i, i^{\prime}} \prod_{j=1}^{j=s}\left(\frac{\delta}{q_{i} q_{i}^{\prime}}\right)_{j}-\cdots$

Aus der Bedeutung des Zeichens $\left(\frac{\delta}{q_{i_{1}} \cdots q_{i_{m}}}\right)_{j}$ ergibt sich unmittelbar die Relation

$$
\left(\frac{\partial}{q_{i_{1}} \cdots q_{i_{m}}}\right)_{j}=\frac{\grave{j}_{j}}{q_{i_{1}} \cdots q_{i_{t}}},
$$

wenn den Primzahlen $q_{i_{1}}, \ldots, q_{i_{m}}$ eine solche Anordnung gegeben wurde, dass die $t$ ersten Zahlen die einzigen dieser Reihe sind, welche in nicht geringerer Potenz in $n_{j}$, wie in $\delta$, aufgehen; die Anzahl $t$ derselben kann natürlich auch gleich Null sein, in welchem Falle der gesuchte größte gemeinsame Theiler gleich $\delta_{j}$ wird.

Führt man den gefundenen Ausdruck (III) in die Gleichung (II) ein, so siebt man, dass im Zähler eines jeden Addenden das 
Product $\prod_{j=1}^{j=s} \delta_{j}$ auftritt, während im Nenner die Primzahl $q_{i}$ $\left(i=1,2^{j}, \ldots, r\right)$ so oftmal bleibt, als die Anzahl $l_{q_{i}}$ derjenigen Zahlen $n_{j}(j=1,2, \ldots, s)$ beträgt, welche den Primfactor $q_{i}$ in nicht geringerer Potenz enthalten, wie der Exponent $\delta$.

Die Gleichung (II) nimmt demnach die Gestalt:

$$
\bar{\varphi}\left(\delta ; n_{1}, \ldots, n_{s}\right)=\prod_{j=1}^{j=s} \delta_{j}\left\{1-\sum_{q} \frac{1}{q^{l} q}+\sum_{q, q^{\prime}} \frac{1}{q^{l} q^{\prime l q^{\prime}}}-\cdots\right\}
$$

an und man ist im Einklange mit dem Satze (3) der Nr. 5 zu dem folgenden Theorem gelangt:

Ist die Zahl $\delta$ in ihre Primfactoren zerlegt gleich dem Producte $\prod_{i=1} q_{i}^{k_{i}}$, so wird die Anzahl der Elemente vom Grade $\delta$ innerhalbeiner Abel'schen Gruppe mit $s$ Basiselementen, deren Gradzahlen bezw. die Werte $n_{1}, \ldots, n_{s}$ besitzen, durch den Ausdruck

$$
\prod_{j=1}^{j=s} \delta_{j} \prod_{i=1}^{i=r}\left(1-\frac{1}{q_{i}^{l} q_{i}}\right)
$$

bestimmt, wo unter $\delta_{j}(j=1,2, \ldots, s)$ der größte gemeinsame Theiler zwischen $\delta$ und $n_{j}$, unter $\dot{l}_{q_{i}}$ $(i=1,2, \ldots, r)$ aber die Anzahl derjenigen der Zahlen $n_{1}, \ldots, n_{s}$ zu verstehen ist, welche den Primfactor $q_{i}$ in nicht geringerer Potenz enthalten, als der Exponent ò.

Die Anzahl $\bar{\varphi}\left(\delta ; n, \ldots, n_{s}\right)$ ist also gleich der jener Zahlen, $j=s$

welche kleiner als II $\delta_{j}$ und durch keine der Potenzen $q_{i}^{l} q_{i}$ $(i=1, \ldots, r)$ theilbar sind, und wird dann und nur dann gleich Null, wenn wenigstens ein Exponent $l_{q_{i}}$ gleich Null ist, d. h. wenigstens. ein Primfactor von $\delta$ ofter in $\delta$, als in einer jeden der Gradzahlen $n_{1}, \ldots, n_{s}$ aufgeht. Hieraus ergibt sich das folgende Theorem:

Es gibt innerhalb der Abel'schen Gruppe H dann und nur dann Elemente vom Grade $\delta$, wenn $\delta$ ein Theiler des kleinsten gemeinsamen Vielfachen $v$ von $n_{1}, \ldots, n_{s}$ ist. 
Die Zahl $v$ ist mithin der höehste Grad, welchen die Elemente von $H$ erreichen und es gibt kein Element vom Grade $n$, wenn nicht je zwei der Zahlen $n_{1}, \ldots, n_{s}$ theilerfremd sind; nur in diesem Falle ist also die Gruppe $\mathrm{H}$ regulär.

Nimmt man nun in der That je zwei der Zahlen $n_{1}, \ldots, n_{s}$ theilerfremd an, so wird für einen jeden Divisor ò von $n$ das Product $\prod_{j=1}^{j=s} \delta_{j}$ gleich $\delta$, die Anzahl $l_{q_{i}}$ aber gleich der Einheit und die Function $\bar{\varphi}\left(\delta ; n_{1}, \ldots, n_{s}\right)$ erhält die Gestalt

$$
\delta \prod_{i=1}^{i=r}\left(1-\frac{1}{q_{i}}\right)
$$

der Euler-Gauss'schen Function $0(0) .1$ )

Somit stellt die Function $\bar{\varphi}\left(\delta ; n_{1}, \ldots, n_{s}\right)$ eine Verallgemeinerung der Function $\varphi(\delta)$ dar und in der That bleiben auch für die erstere Function die beiden Hauptsätze der letzteren bestehen:

Sind die beiden Zahlen $\delta$ und $\delta^{\prime}$ theiler(3) fremd, so besteht die Gleichung

$$
\bar{\varphi}\left(\delta ; n_{1}, \ldots, n_{s}\right) \bar{\varphi}\left(\delta^{\prime} ; n_{1}, \ldots, n_{s}\right)=\bar{\varphi}\left(\delta \delta^{\prime} ; n_{1}, \ldots, n_{s}\right)
$$

und

Erstreckt sich die Summe $\sum_{\delta} \bar{\varphi}_{j=s}\left(\delta ; n_{1}, \ldots, n_{s}\right)$ aut (4) alle Theiler $\delta \underset{\substack{j=s \\ j=s}}{\text { Productes }} \prod_{j=1} n_{j}$, soerhält man das Product $\prod_{j=1} n_{j}$ selbst.

Der Satz (3) folgt unmittelbar aus der im Theoreme (1) angegebenen Form der Function $\bar{\varphi}\left(\delta ; n_{1}, \ldots, n_{s}\right)$; der Satz (4) hingegen aus der Bedeutung der Anzahl $\varphi\left(\delta ; n_{1}, \ldots, n_{s}\right)$ : Jedes Element der Gruppe $H$ besitzt einen und nur einen Grad, welcher die Gradzahl von $\mathrm{H}$, nämlich $\prod_{j=1}^{j=s} n_{j}$ theilt, wird also einmal und nur einmal in der Summe $\sum_{\delta} \bar{\varphi}\left(\hat{o} ; n_{1}, \ldots, n_{s}\right)$ gezählt. Übrigens braucht gemäß dem Satze (2) diese Summe bloß auf alle Theiler des kleinsten gemeinsamen Multiplums der Zahlen $n_{1}, \ldots, n_{s}$ erstreckt zu werden.

1) Die Specialisierung $s=1$, welche das nämliche Resultat ergibt, ist natïrlich nur nnwesentlich von der $\mathrm{i}$. T. angegebenen unterschieden. 
Dividiert man eine der Zahlen $n_{1}, \ldots, n_{s}$, z. B. $n_{s}$ durch einen ihrer Primfactoren $q$, so wird die Function $\bar{\varphi}\left(\delta ; n_{1}, \ldots, n_{s-1}, \frac{n_{s}}{q}\right)$ gleich $\bar{\varphi}\left(\hat{\delta} ; n_{1}, \ldots, n_{s}\right)$, oder gleich $\frac{1}{q} \bar{\varphi}\left(\hat{\delta} ; n_{1}, \ldots, n_{s}\right)$,

$$
\text { oder gleich } \frac{1-\frac{1}{q^{l_{q}-1}}}{q\left(1-\frac{1}{q^{l_{q}}}\right)} \bar{\varphi}\left(\hat{\delta} ; n_{1}, \ldots, n_{s}\right) \text {, }
$$

je nachdem die Zahl $n_{s}$ den Primfactor $q$ in höherer, oder niedrigerer, oder in derselben Potenz, wie der Exponent $\delta$ enthält.

Speciell folgt aus der ersten Gleichung der Satz:

Die Function $\bar{\varphi}\left(\delta ; n_{1}, \ldots, n_{s}\right)$ bleibt ungeändert, (5) wenn man die Zahlen $n_{i}(i=1, \ldots, s)$ mit irgend welchen zu o theilerfremden Zahlen multipliciert oder dividiert.

Die beiden Sätze (3) und (5) gestatten den Satz (4) auf arithmetischem Wege abzuleiten.

Auf Grund des Satzes (3) erhält man nämlich, wenn das kleinste gemeinsame Vielfache $v$ von $n_{1}, \ldots, n_{s}$ in seine Primfactoren zerlegt gleich dem Producte $\prod_{i=1}^{i=r} p_{i}^{m_{i}}$ ist, die Gleichung

$$
\sum_{\delta} \bar{\varphi}\left(\delta ; n_{1}, \ldots, n_{s}\right)=\prod_{i=1}^{i=r} \sum_{\sigma_{i}=0}^{m_{i}} \bar{\varphi}\left(p^{\sigma_{i}} ; n_{1}, \ldots, n_{s}\right),
$$

in welcher das $\delta$ alle Theiler von $v$ zu durchlaufen hat.

aber vermöge des Salzes (5) in die Form

Eine jede rechts auftretende Summe $\sum_{\sigma=0}^{n} \bar{\varphi}\left(p^{\alpha} ; n_{1}, \ldots, n_{s}\right)$ kann

$$
\sum_{\sigma=0}^{m} \bar{\varphi}\left(p^{\sigma} ; p^{\alpha_{1}}, \ldots, p^{\alpha_{i}}\right)
$$

gebracht werden, wo die nach der Kürzung eventuell auftretenden Zahlen $v_{i}=1$ unterdrückt und die Potenzen $p^{\alpha_{i}}$ ihrer Gröfe nach geordnet: $p^{\alpha_{1}} \geq p^{\alpha_{2}} \geq \cdots \geq p^{\alpha_{t}}$ gedacht sind; natürlich muss dann der höchste Exponent $\alpha_{1}$ gleich $m$ sein. Bezeichnet man nun mit

$$
\mu_{\sigma}(\sigma=1,2, \ldots, m)
$$


die Anzahl der Zahlen $\alpha_{1}, \ldots, \alpha_{t}$, welche nicht kleiner als $\sigma$ sind, so ergeben sich die Gleichungen

$$
\bar{\varphi}\left(p^{\sigma} ; p^{\alpha_{1}}, \ldots, p^{\alpha_{t}}\right)=p^{\mu_{\sigma} \sigma+\alpha_{a_{\sigma}}+1}+\ldots+\alpha_{t}\left(1-\frac{1}{p^{\mu_{\sigma}}}\right)(\sigma=1, \ldots, m)
$$

in Übereinstimmung mit dem Satze (2) der Nr. 2. Hierbei ist natürlich für die Summe $\alpha_{\mu_{\sigma}+1}+\cdots+\alpha_{t}$ im Falle, dass $\mu_{\sigma}=t$ ist, Null zu setzen, ein Fall, der stets für $\sigma=1$ eintritt.

Nach der Definition von $\mu_{j}$ haben genau $\mu_{\sigma-1}-\mu_{\sigma}$ der Zahlen $\alpha_{1}, \ldots, \alpha_{t}$ den $W$ ert $(\sigma-1)$, so dass die Summe $\alpha_{\mu_{\sigma}+1}+\cdots+\alpha_{t}$ den Wert $\left(\mu_{\sigma-1}-\mu_{\sigma}\right)(\sigma-1)+\left(\mu_{\sigma-2}-\mu_{\sigma-1}\right)(\sigma-2)+\cdots+\left(\mu_{1}-\mu_{2}\right) \cdot 1$, d. h. $-\mu_{\sigma} \sigma+\sum_{j=1}^{\sigma} \mu_{j}$ besitzt and die Function $\bar{\varphi}\left(p^{\sigma} ; p^{\alpha_{1}}, \ldots, p^{\alpha_{\ell}}\right)$ die einfache Gestalt

annimmt.

$$
p^{\sum_{j}^{\sigma} \mu_{j}}\left(1-\frac{1}{p^{\mu_{\sigma}}}\right)
$$

Man erhält also die Gleichung

$$
\sum_{\sigma=0}^{m} \bar{\varphi}\left(p^{\sigma} ; p^{\alpha_{1}}, \ldots, p^{\alpha_{t}}\right)=1+\left(p^{t}-1\right)+\sum_{\sigma=2}^{m}\left(p^{j=1}-p^{\sum_{j=1}^{\sigma} \mu_{j}}\right) \text {. }
$$

Auf der rechten Seite derselben bleibt aber nur das letzte positive Glied $p^{\sum_{j=1}^{m}{ }^{\prime \prime} j}$ Umformung auch das Product

$$
\prod_{\bar{i}}^{j=t} p^{\alpha_{j}}
$$

gesetzt werden kann, weil die Summe $\alpha_{1}+\cdots+\alpha_{t}$ sich in der Form $\mu_{m} m+\alpha_{\mu_{m}+1}+\cdots+\alpha_{t}$ schreiben lässt.

Das Product aller dieser Producte über alle Primtheiler $p$ von $v$ genommen, ist aber gleich dem Producte $\prod_{i=1}^{i=s} n_{i}$, wie es der Satz (4) verlangt.

Betrachtet man neben der Function $\bar{\varphi}\left(\hat{0} ; n_{1}, \ldots, n_{s}\right)$ die Function $\bar{\varphi}\left(\delta ; n_{1}^{\prime}, \ldots, n_{s^{\prime}}^{\prime}\right)$, wobei unter $n_{1}^{\prime}, \ldots, n_{s^{\prime}}^{\prime}$ die Gradzahlen der Basiselemente einer neuen Gruppe $\mathrm{H}^{\prime}$ zu verstehen sind, so er- 
hält man, falls die Zahlen $l_{q}^{\prime}$ für diese zweite Function die Rolle der Zahlen $l_{q}$ bei der ersteren vertreten, ohne Weiteres die Gleichung:

$$
\begin{gathered}
\bar{\varphi}\left(\delta ; n_{1}, \ldots, n_{s}\right) \bar{\varphi}\left(\delta ; n_{1}^{\prime}, \ldots, n_{s}^{\prime}\right)= \\
=\bar{\varphi}\left(\delta ; n_{1}, \ldots, n_{s}, n_{1}^{\prime}, \ldots, n_{s}^{\prime}\right) \mathbf{I I}_{q} \frac{\left(1-\frac{1}{q^{l_{q}}}\right)\left(1-\frac{1}{q_{s}^{\prime}}\right)}{1-\frac{1}{q^{\prime}+i_{q}^{\prime}}},
\end{gathered}
$$

in der das Productzeichen sich auf alle die Primfactoren der Grade $n_{j}$ und $n_{j^{\prime}}^{\prime}$ zu beziehen hat, für welche gleichzeitig Zahlen $n_{j}$ $(j=1,2, \ldots, s)$ and $n_{j^{\prime}}^{\prime}\left(j^{\prime}=1,2, \ldots, s^{\prime}\right)$ existieren, die in nicht geringerer Potenz den Primtheiler $q$ enthalten, wie der Exponent $\delta$.

Ist speciell jede der Zahlen $n_{1}, \ldots, n_{s}$ theilerfremd mit allen Graden $n_{1}^{\prime}, \ldots, n_{s^{\prime}}^{\prime}$, so folgt hieraus die einfache Gleichung

$$
\bar{\varphi}\left(0 ; n_{1}, \ldots, n_{s}\right) \bar{\varphi}\left(\hat{\delta} ; n_{1}^{\prime}, \ldots, n_{s^{\prime}}^{\prime}\right)=\bar{\varphi}\left(\delta ; n_{1}, \ldots, n_{s}, n_{1}^{\prime}, \ldots, n_{s^{\prime}}^{\prime}\right) .
$$

Schließlich sei noch der Fall hervorgehoben, dass die Zahl $\delta$ gemeinsamer Theiler aller der Grade $n_{1}, \ldots, n_{s}$ ist; dann erhält man für die Anzahl der Elemente vom Grade $\delta$ innerhalb der Gruppe $H$ das Product $\delta \prod_{i=1}^{i=r}\left(1-\frac{1}{q_{i}^{s}}\right)$, welches bekanntlich die Anzahl der s-zahligen Zahlsysteme angibt, deren Angehörige die Grenze $\delta$ nicht überschreiten und einen zu $\delta$ theilerfremden größten gemeinsamen Divisor besitzen. Der Grund dieser Thatsache wird übrigens in der nächsten Nummer klar zu Tage treten.

Aus dem Ausdrucke (I) kann man nicht nur die Anzahl, sondern auch das Produet $\overline{\mathfrak{P}}_{\delta}$ aller Elemente vom. Grade õ innerhalb der Gruppe $\mathrm{H}$ ableiten, wenn man nur einen jeden Complex $\mathrm{H}_{\tau}$ durch das Product $\mathfrak{F}_{\tau}$ aller seiner Elemente und die Symbole $\dot{f}$ und - durch das Multiplications-, bezw. Divisionszeichen ersetzt.

Der Ausdruck (I) nimmt dann die Form

$$
\begin{aligned}
& \mathfrak{P}_{\delta} \text { II } \mathfrak{F}_{\delta} \ldots \\
& \frac{\frac{q q^{\prime}}{q q^{\prime}}}{\coprod_{q} \Re_{\frac{\delta}{q}} \prod_{q q^{\prime} q^{\prime \prime}} \mathfrak{W}_{\frac{\delta}{q q^{\prime} q^{\prime \prime}}}}
\end{aligned}
$$

an. Da das Product $\Re_{\frac{\delta}{q_{i_{1}} \ldots} \overline{q_{i_{t}}}}$ nach den Ausführungen der vorigen Nummer davon abbängt, ob eine einzige unter den s-Zablen 
$\left(\frac{\delta}{q_{i_{1}} \ldots q_{i_{l}}}\right)_{j}(j=1,2, \ldots, s)$ gerade ist oder nicht, besteht im Falle, dass die Zahl o ungerade oder durch 4 theilbar vorausgesetzt wird die Gleichung

$$
\mathfrak{F}_{\delta}=\mathfrak{P} \frac{\delta}{\overline{q_{i_{1}} \cdots} \bar{q}_{i_{t}}},
$$

so dass sich der Ausdruck (I) auf die Einheit reduciert.

Diese Gleichung bleibt auch noch unter der Annahme $\delta=2(2 \mu+1)(\mu=1,2,3 \ldots)$ erhalten, wenn für $q_{i_{1}}, \ldots, q_{i_{t}}$ lauter ungerade Primfactoren von $\delta$ gesetzt werden. Andernfalls ist der Quotient $\frac{\hat{o}}{q_{i_{1}} \cdots q_{i_{t}}}$ ungerade, also $\frac{\mathfrak{P} \frac{\delta}{q_{i_{1}} \cdots q_{i_{t}}}}{}=1$.

Es tritt somit auch in diesem Falle das Product $\mathfrak{P}_{\delta}$ ebenso oft im Zähler, wie im Nenner des Ausdruckes (I) auf und man erhält wieder die Beziehung

$$
\overline{\mathfrak{P}_{\delta}}=1 \text {. }
$$

Nur wenn die Zahl ò gleich 2 ist, ergibt sich für $\overline{\mathfrak{F}}_{2}$ oder $\mathfrak{P}_{\mathrm{z}}$ das Element $B_{i}^{\frac{n_{i}}{2}}$ oder die Einheit, je nachdem $B_{i}$ das einzige Basiselement mit geradem Grade ist oder nicht. ${ }^{1}$ )

Man ist somit zu dem folgenden Satze gelangt:

Iultipliciert man alle einer Abel'schen (6) Gruppe angehorigen Elemente vom Grade $\delta$ mit einander, so erhält man stets die Einheit, falls $\delta$ größer als 2 ist.

Als Beispiel möge hier der schon früher herangezogene Fall dienen, dass die Gruppe $\mathrm{H}$ aus der Gesammtheit der $n^{\text {ten }}$ Einheitswurzeln und der Einheit gebildet sei. Setzt man dann in dem Ausdrucke (I) an Stelle des Complexes $\mathrm{H}_{\delta} \delta$ das Product der

betreffenden Wurzelfactoren, d. h. die Differenz $\left(\frac{\delta}{x^{\overline{q_{i_{1}} \cdots q_{i_{t}}}}-1}\right)$, so erbält man, wenn außerdem für das Zeichen $\dot{+}$, bezw. $\doteq$ das Multiplications-, bezw. das Divisionszeichen genommen wird, für das Product aller derjenigen Wurzelfactoren, deren Wurzeln erst

1) Vergl. Satz (6) der Nr. 6. 
anf die $\delta^{\text {te }}$ Potenz erhoben gleich der Einheit werden, den aus der Theorie der Kreistheilung bekannten irreducibelen Factor

$$
\frac{\left.\left(x^{\delta}-i\right) \prod_{q q^{\prime}}^{\frac{\delta}{q q^{\prime}}}-1\right) \ldots}{\prod_{q}\left(x^{\frac{\delta}{q}}-1\right) \ldots}
$$

\section{Nr. 8. Über eine Verallgemeinerung der Function $\bar{\varphi}\left(\hat{o} ; n_{1}, \ldots, n_{s}\right)$.}

Im Folgenden soll die Anzahl der Elemente vom Grade $\delta$ innerbalb der Gruppe $\mathrm{H}$ anf einem zweiten Wege ermittelt werden, der vielleicht darum nicht ganz des Interesses entbehrt, weil er für die Function $\bar{o}\left(\hat{o} ; n_{1}, \ldots, n_{s}\right)$ eine andere Bedeutung und damit zugleich den Fingerzeig zu einer Verallgemeinerung derselben liefern wird. Freilich kommt auch bei dieser Überlegung in letzter Linie die Methode des Ausscheidens und Hinzufügens in Anwendung.

Der Grad $\gamma$ des Elementes

$$
S=\prod_{j=1}^{j=s} B_{j}^{\alpha_{j}^{\prime}}
$$

muss die Bedingung $\alpha_{j}^{\prime}=\dot{\lambda}_{j}^{\prime} n_{j}$, d. h.

$$
\gamma=\lambda_{j} \frac{n_{j}}{\nu_{j}}
$$

für $j=1,2, \ldots, s$ erfüllen, wo unter $v_{j}$ der grölte gemeinsame Theiler ron $n_{j}$ und $\alpha_{j}^{\prime}$, unter $\lambda_{j}^{\prime}$, bezw. $\lambda_{j}$ aber eine ganze Zahl zu verstehen ist. Der Grad $\gamma$ muss also ein Multiplum des kleinsten gemeinsamen Vieltachen $v$ der $s$ Zahlen $\frac{n_{1}}{v_{1}}, \ldots, \frac{n_{s}}{v_{s}} \operatorname{sein}$ und zwar ist er gleich $v$, weil die Gleichung $S^{v}=1$ besteht.

Wählt man also speciell für $S$ ein Element, welches der Gruppe $\mathrm{H}_{\delta}$ angehört, mithin nach dem Ausdrucke (I) der $\mathrm{Nr} .6$ die Form

$$
\prod_{j=1}^{j=s} B_{j}^{a_{j}} \frac{n_{j}}{\delta_{j}}\left(\boldsymbol{\alpha}_{j}=1,2, \ldots, \delta_{j}\right)
$$

besitzt, so erhält man für $\nu_{j}$, den $\operatorname{Wert} \frac{n_{j} \tau_{j}}{\delta_{j}}(j=1,2, \ldots, s)$, wobei mit $\tau_{j}$ das größte gemeinsame $\mathrm{Mah}$ der Zahlen $\alpha_{j}$ und $\delta_{j}$ bezeichnet ist; und der Grad $\gamma$ des betrachteten Elementes wird der 
durchgeführten Überlegung zufolge gleich dem kleinsten gemeinsamen Vielfachen der $s$ Zahlen $\frac{\delta_{1}}{\tau_{1}}, \ldots, \frac{\delta_{s}}{\tau_{s}}$.

Soll demnach der Grad irgend eines Elementes von der Form (I) gleich $\delta$ werden, so leuchtet unmittelbar ein, dass jeder Primfactor von $\delta$ wenigstens in einer der Zahlen $\delta_{1}, \ldots, \delta_{s}$ in derselben Potenz aufgehen muss, wie in $\delta$; andernfalls existiert überhaupt kein der Gruppe $H$ angehöriges Element vom Grade $\delta$; ein Resultat, das vollständig mit den Ausführungen der vorigen Nr. im Einklange steht.

Angenommen, diese Forderung sei ertüllt, so entsteht die Frage: Welche unter den $\delta_{1}, \delta_{2} \ldots \delta_{s} s$ zahligen Wertesystemen

$$
\alpha_{1}, \alpha_{2}, \ldots, \alpha_{s}\left(\begin{array}{c}
\alpha_{j}=1,2, \ldots, \hat{o}_{j} \\
j=1,2, \ldots, s
\end{array}\right)
$$

haben die Eigenschaft, dass das kleinste gemeinsame Vielfache der zu jedem solchen Systeme gehörigen $s$ Zahlen $\frac{\delta_{1}}{\tau_{1}}, \ldots, \frac{\delta_{s}}{\tau_{s}}$ gleich $\delta$ wird?

Es seien

$$
\delta_{j}^{(i)}, \delta_{j}(i), \ldots, \delta_{j_{i}(i)}
$$

genau diejenigen der Zahlen $\delta_{1}, \delta_{2}, \ldots, \delta_{s}$, welche den Primfactor $q_{i}$ von $\delta$ in derselben Potenz, wie der Exponent $\delta$, enthalten; dann ist klar, dass das kleinste gemeinsame Vielfache von $\frac{\delta_{1}}{\tau_{1}}, \ldots, \frac{\delta_{s}}{\tau_{s}}$ dann und nur dann den Factor $q_{i}$ in der nämlichen Potenz, wie $\delta$, enthält, wenn wenigstens eine der Zahlen

$$
\tau_{j_{1}^{(i)}}, \tau_{j_{2}^{(i)}}, \ldots, \tau_{j_{i}^{(i)}}
$$

mit $q_{i}$ theilerfremd ist, $d$. $h$. wenn das System $\alpha_{1}, \alpha_{2}, \ldots, \alpha_{s}$ so gewählt wurde, dass das gröfte gemeinsame $\mathrm{Ma}$ des in ihm enthaltenen Theilsystemes

$$
\alpha_{j_{1}^{(i)}}, \alpha_{j_{2}^{(i)}}, \ldots, \alpha_{j_{l_{i}}^{(i)}}
$$

durch $q_{i}$ nicht theilbar ist.

Findet dies für alle verschiedenen Primfactoren $q_{1}, \ldots, q_{r}$ von $\delta$ statt, so besitzt das Element

$$
\prod_{j=1}^{j=s} B_{j}^{\alpha_{j}} \frac{n_{j}}{\delta_{j}}
$$

thatsächlich den Grad $\delta$. 
Um also alle jene Systeme $\alpha_{1}, \ldots, \alpha_{s} z u$ finden, welche $z u$ den Elementen vom Grade $\delta$ gehören, hat man bloß aus allen möglichen Systemen

$$
\alpha_{1}, \alpha_{2}, \ldots, \alpha_{s}\left(\begin{array}{c}
\alpha_{j}=1,2, \ldots, \delta_{j} \\
j=1,2, \ldots ; s
\end{array}\right)
$$

diejenigen auszuscheiden, bei welchen die Theilsysteme $\alpha_{j_{1}^{(i)}}, \ldots, x_{j_{l_{i}}^{(i)}}$ $(i=1,2, \ldots, r)$ einen größten gemeinsamen Theiler, der den Primfactor $q_{i}$ enthält, besitzen und daher von der Form

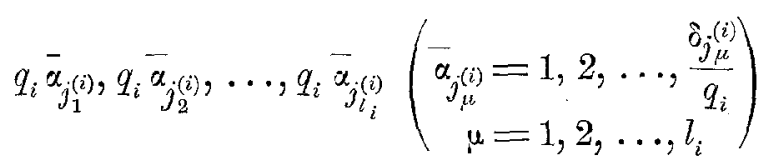

für $i=1,2, \ldots, r$ sind.

Obne größere Mühe kann diese Aufgabe mit Hilfe der Methode des Ausscheidens und Hinzufügens gleich ganz allgemein gelöst werden:

Aus dem Complexe $T$ aller möglichen $s$ zahligen positiven Zahlsysteme

$$
\alpha_{1}, \alpha_{2}, \ldots, \alpha_{s}\left(\begin{array}{l}
\alpha_{j}=1,2,3, \ldots \\
j=1,2, \ldots, s
\end{array}\right)
$$

sollen alle verschiedenen Zahlsysteme der $r$ speciellen Complexe $T_{\imath}$

$$
n_{i}^{(1)} \alpha_{11}, n_{i}^{(2)} \alpha_{2}, \ldots, n_{i}^{(s)} \alpha_{s} \quad(i=1,2, \ldots, r)
$$

ausgeschieden werden, wobei unter $n_{i}^{(1)}, \ldots, n_{i}^{(s)}$ beliebige ganze positive Zahlen, die auch gleich der Einheit sein können, zu verstehen sind.

Man erkennt ohne Schwierigkeit, dass der den Systemcomplexen $T_{i_{1}}, T_{i_{2}}, \ldots, T_{i_{t}}$ gemeinsame Complex $T_{i_{1}, \ldots, i_{t}}$ die Form

$$
\left(n_{i_{1}}^{(1)}, n_{i_{2}}^{(1)}, \ldots, n_{i_{t}}^{(1)}\right) \alpha_{1}, \ldots,\left(n_{i_{1}}^{(s)}, n_{i_{2}}^{(s)}, \ldots, n_{i_{t}}^{(s)}\right) \alpha_{s}
$$

besitzen muss, wo mit dem Symbol $\left(n_{i_{1}}^{(j)}, n_{i_{2}}^{(j)}, \ldots, n_{i_{t}}^{(j)}\right)$ das kleinste gemeinsame Vielfache der $t$ Zahlen $n_{i_{1}}^{(j)}, n_{i_{2}}^{(j)}, \ldots, n_{i_{t}}^{(j)}$ für $j=1,2, \ldots, s$ bezeichnet ist, und man erhält auf Grund des Ausdruckes (I) der Nr. 1 im I. Abschn. den gesuchten Complex in der Form

$$
T \cdot \dot{\Sigma} T_{i}+\dot{\Sigma} T_{i, i^{\prime}} T_{i, i^{\prime}} \dot{-} \ldots
$$


Bricht man also die laufenden Zahlen $\alpha_{1}, \ldots, \alpha_{s}$, bezw. bei den beliebig gewählten Grenzen $\gamma_{1}, \ldots, \gamma_{s}$ ab und zählt man nur die in jedem Complexe enthaltenen Systeme, so ergibt sich als Anzahl derjenigen Wertsysteme, welche wohl dem Complexe

$$
a_{1}, \ldots, \alpha_{s}\left(\begin{array}{l}
a_{j}=1,2, \ldots, \gamma_{j} \\
j=1,2, \ldots, s
\end{array}\right)
$$

angehören, aber nicht die Form (II) besitzen, der Ausdruck

(III)

$$
\begin{gathered}
\prod_{j=1}^{j=s} \gamma_{j}-\sum_{i} \prod_{j=1}^{j=s}\left[\frac{\gamma_{j}}{n_{i}^{(j)}}\right]+ \\
+\sum_{i, i^{\prime}} \prod_{j=1}^{j=s}\left[\frac{\gamma_{j}}{\left(n_{i}^{(j)}, n_{i^{\prime}}^{(j)}\right)}\right]-\sum_{i, i^{\prime}, i^{\prime \prime}} \prod_{j=1}^{j}\left[\frac{\gamma_{j}}{\left(n_{i}^{(j)}, n_{i^{\prime}}^{(j)}, n_{i^{\prime \prime}}^{(j)}\right.}\right]+\cdots
\end{gathered}
$$

Man ist damit zu einer weitgehenden Verallgemeinerung der Euler-Gauss'schen Function $o(\gamma)$ gelangt, welche auch die in der Nr. 2 des I. Abschn. angegebene Erweiterung umfasst.

Setzt man in dem Ausdrucke (III) erstens an die Stelle von $n_{i}^{(j)}(j=1, \ldots, s)$ den Primfactor $q_{i}$ oder die Einheit, je nachdem der Index $j$ gleich einer der Zahlen $j_{1}^{(i)}, \ldots, j_{l_{i}}^{(i)}$ ist oder nicht, für jeden Wert $i=1,2, \ldots, r$ und nimmt man ferner für $\gamma_{j}$ die Zahl $\delta_{j}(j=1,2, \ldots, s)$, so geht der Ausdruck (III) in die Function $\bar{\varphi}\left(\delta ; n_{1}, \ldots, n_{s}\right)$ über.

Zweitens soll der specielle Fall

$$
n_{i}^{(1)}=n_{i}^{(2)}=\ldots=n_{i}^{(s)}=n_{i}
$$

für $i=1,2, \ldots, r$ betrachtet werden, wobei überdies je zwei der Zahlen $n_{1}, \ldots, n_{r}$ als theilerfremd angenommen werden; ferner möge unter $N$ ein positives Multiplum der Zahlen $n_{1}, \ldots, n_{r}$, unter $m_{j}(j=1,2, \ldots, s)$ aber irgend ein Wert verstanden werden, welcher der Bedingung

Genüge leistet.

$$
\frac{N}{\gamma_{j}} \geqq m_{j}>\frac{N}{1+\gamma_{j}}
$$

Unter diesen Voraussetzungen nimmt der Ausdruck (III) die Gestalt

(IV) $\prod_{j=1}^{j=s}\left[\frac{N}{m_{j}}\right]--\sum_{i} \prod_{j=1}^{j=s}\left[\frac{N}{m_{j} n_{i}}\right]+\sum_{i, i^{\prime}} \prod_{j=1}^{j=s}\left[\frac{N}{m_{j} n_{i} n_{i^{\prime}}}\right]-\cdots$ 
an and bestimmt die Anzahl o ${ }^{*}\left(m_{1} \ldots m_{s}\right)(N)$ derjenigen Zahlsysteme

$$
a_{1}, \ldots, x_{s}\left(\begin{array}{c}
\alpha_{j}=1,2, \ldots\left[\frac{N}{m_{j}}\right] \\
j=1,2, \ldots, s
\end{array}\right),
$$

deren größter gemeinsamer Theiler weder durch $n_{1}$, noch durch $n_{2}, \ldots$, noch durch $n_{s}$ theilbar ist.

Der Ausdruck (IV) besitzt die Form der rechten Seite der Gleichung (III) der Nr. 2 im I. Abschn. und es gilt auf Grund des Umkehrproblemes, d. h. der Gleichung (II) l. c. der Satz

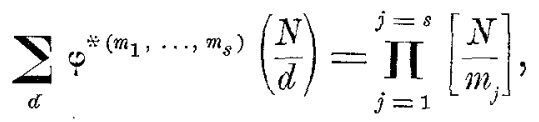

wo für $d$ alle Theiler von $N$ der Form $\prod_{i=1}^{i=r} n_{i}^{k_{i}}\left(k_{i}=0,1,2, \ldots\right)$ zu setzen $\sin d$.

Nimmt man endlich für $n_{1}, \ldots, n_{s}$ die verschiedenen Primfactoren von $N$, so geht die Function $\varphi^{*\left(m_{1}, \ldots, m_{s}\right)}(N)$ in die Function $\left.\varphi^{\left(n_{1}\right.}, \ldots, m_{s}\right)(N)$ iiber, welche die Anzahl der Zahlsysteme (V) angibt, deren größter gemeinsamer Theiler mit $N$ theilerfremd ist. ${ }^{1}$ )

1) Man vergl, bezüglich der Function $\left.\varphi^{\left(m_{1}\right.}, \ldots, m_{e}\right)(N)$ : Ableitung zahlenth. Relationen etc. von Herrn R. Daublebsky v. Sterneck, Monatsh. f. Mathem. u. Physik, V., p. 255 u. f.

Die dort aufgestellte Beziehung

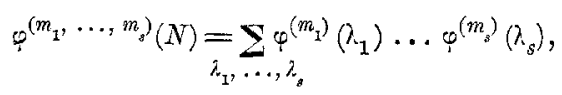

in der die Summe sich auf alle Wertesysteme $\lambda_{1}, \ldots, \lambda_{s}$ zu erstrecken hat, deren kleinstes gemeinsames Vielfache gleich $N$ ist, gilt in entsprechender Weise auch für die allgemeinere Function $\varphi^{*\left(m_{1}, \ldots, m_{s}\right)}(\boldsymbol{N})$.

Versteht man nämlich unter den ganzen Zahlen $d, \lambda_{1}, \ldots, \lambda_{s}$ stets solche $i=r$

Theiler von $N$, welche die Form $\prod n_{i}^{k_{i}}$ besitzen und betrachtet man die Function $i=1$

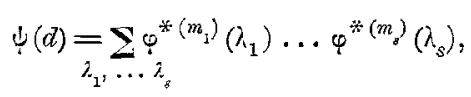

wo die Summe sich aut alle möglichen Systeme $\lambda_{1}, \ldots, \lambda_{3}$ zu erstrecken hat, deren kleinstes gemeinsames Vielfache gleich $d$ ist, so erkennt man auf Grüd der Relation

$$
\sum_{a} \varphi^{*\left(m_{j}\right)}(d)=\left[\frac{N}{m_{j}}\right] \quad(j=1,2, \ldots, s)
$$



gehoben:

Schließlich sei noch der folgende allgemeine Satz hervor-

Multipliciert man den Ausdruck (III) mit dem analogen, den man erhält, wenn man alle Buchstaben accentuiert, so ergibt sich wieder ein analog gebauter Ausdruck.

Derselbe stellt die Anzahl der $s+s^{\prime}$ zahligen Zahlsysteme des Complexes $T+T^{\prime}$ dar, welche nicht den $r+r^{\prime}$ Complexen $T \nmid T_{i^{\prime}}^{\prime}\left(i^{\prime}=1,2, \ldots, r^{\prime}\right)$ und $T_{i} \dagger T^{\prime \prime}(i=1,2, \ldots, r)$ angehören, wobei unter $T$ und $T_{i}$ die oben angeführten, nunmehr jedoch entsprechend begrenzten Complexe, unter $T^{\prime}$ und $T_{i^{\prime}}^{\prime}$ aber die analogen Gebilde zu verstehen sind.

\section{Nr. 9. Über die Multiplicatoren eines Systemes von Elementen.}

Nachdem in der Nr. 6 bereits zwei specielle Arten von Untergruppen untersucht worden sind, wenden wir uns der Betrachtung einer dritten Art zu, welche in der folgenden Weise gewonnen werden kann :

Es sei :

$$
A_{1}, A_{2}, \ldots, A_{k}
$$

ein System von irgend welchen $k$ der Gruppe $H$ angehörigen Elementen und es sei $M$ ein Element von H, welches die Eigenschaft besitzt, dass das System

$$
M A_{1}, M A_{2}, \ldots, M A_{k},
$$

wenn auch in geänderter Reihenfolge mit dem Systeme (I) vollständig übereinstimmt. Ohne weiteres erkennt man, dass die Gesammtheit aller derart definierten Elemente $M$ eine Untergruppe von $\mathrm{H}$ bildet, welche als Multiplicatorengruppe des Systemes. (I) bezeichnet werden soll, während ein Element derselben Multiplicator des Systemes (I) genannt werden möge.

(vergl. Gleichung (VI)) durch sucessive Summation mach $\lambda_{1}, \ldots, \lambda_{s}$ die Richtigkeit der Beziehung

$$
\sum_{d} \downarrow(d)=\prod_{j=1}^{j=8}\left[\frac{N}{m_{j}}\right] .
$$

Und zwar hat sich hier, wie in der unmittelbar vorangehenden Gleichung die Sumpme auf alle Theiler $d$ von $N$ der Form $\prod^{i} n_{i}^{k_{i}}$ zu beziehen.

Wendet man anf die Relation (VIII) das Umkehrproblem (vergl. a. a. 0. Gleichung (II) und (III)) an, so erhält man für $\downarrow(N)$ den Ausdruck (IV), d. h. die Function $\varphi^{*\left(m_{1}, \ldots, m_{s}\right)}(\boldsymbol{N})$, so dass die Gleichung (VII) die gesuchte Beziehung darstellt. 
Bezeichnet man die Elemente der Multiplicatorengruppe der Reihe (I) mit

$$
1, M_{1}, M_{2}, \ldots, M_{s-1} \text {, }
$$

so muss das System (I) neben dem Individuum $A_{1}$ auch das Product $A_{1} M_{i}(i=1,2, \ldots, \overline{s-1})$ enthalten. Alle diese $s$ verschiedenen Elemente mögen zu einer Klasse

$$
A_{1}, A_{1} M_{1}, \ldots, A_{1} M_{s-1}
$$

vereinigt werden.

Enthält diese Klasse das Element $A_{2}$ vom Systeme (I) nicht, so gelangt man auf dieselbe Weise zu einer zweiten Klasse von Individuen

$$
A_{2}, A_{2} M_{1}, \ldots, A_{2} M_{s-1},
$$

welche alle im Systeme (I) enthalten sind und sich sowohl untereinander, als auch von den Elementen der 1. Klasse unterscheiden.

In dieser Weise fortfahrend erkennt man, dass sich das System (I) in $\frac{k}{s}$ Klassen zu je $s$ Elementen theilen muss und man ist somit zu dem folgenden Theorem gelangt:

Der Grad der Multiplicatorengruppe eines: (1) Systemes ist ein Theiler der Anzahl der Elemente dieses Systemes.

Nimmt man speciell für das System (1) selbst eine Gruppe, so ist diese identisch mit ihrer Multiplicatorengruppe; denn der Grad der letzteren kann weder kleiner sein als der Grad der ersteren, weil jedes Element einer Gruppe auch ein Multiplicator der Gruppe ist, noch kann er ihn dem Theoreme (1) zutolge je. übertreffen.

Ferner ergibt sich aus dem Theoreme (1) unmittelbar der Satz:

Die Anzahl der Elemente eines Systemes ist ein Multiplum. des Grades eines jeden Multiplicators desselben.

Ist also die Zahl $k$ mit dem Grade $n$ von $H$ theilerfremd, so existiert kein System von $k$ Zahlen, welches einen von der Einheit verschiedenen Multiplicator besitzt.

Ebenso leicht lässt sich auch die Richtigkeit des folgenden Satzes erkennen:

Ein System von $k$ Zahlen bildet, wenn $k$ Primzahl ist, dann und nur dann eine Gruppe, wenn es einen von der Einheit verschiedenen Multiplicator enthält.

Unter den getroffenen Voraussetzungen kann nämlich die Mrltiplicatorengruppe, weil sie neben der Einheit mindestens noch ein Element enthält, gemäß dem Satze (1) nur den Grad $k$ haben, das System muss also die in diesem Falle reguläre Multiplicatorengruppe enthalten, also mit dieser identisch werden.

Monatsh. f. Mathematik u. Physik. VII. Jahrg. 
Wählt man speciell für $\mathrm{H}$ eine reguläre Gruppe und setzt man den Grad der Multiplicatorengruppe $s$ gleich $\frac{n}{\delta}$, so ist diese entsprechend dem Satze (4) der Nr. 5 nichts anderes, als die Untergruppe der $\delta^{\text {ten }}$ Potenzen von $\mathrm{H}$ und die eben angeführten Klassen des Systemes (I) stimmen vollständig mit den entsprechenden Klassen des Schemas $\left(\mathfrak{H}_{\delta}\right)$ in der Nr. 6 überein, talls man nur dort die Zahl $\delta$ als Theiler von $n$ voraussetzt.

Umgekehrt ist klar, dass beliebige Klassen des Schemas $\left(\mathfrak{A}_{\delta}\right)$ vereinigt ein System von Elementen geben, welches eine Multiplicatorengruppe besitzt, die entweder identisch mit der Klasse (0) ist, oder dieselbe doch als Untergruppe enthält.

Es soll zunächst die Frage, wie diese Klassen zu wählen sind, damit der erstere Fall eintrete, beantwortet und im Anschlusse hieran das Problem gelöst werden, alle der Gruppe $H$ angehörigen Elementensysteme aufzustellen, welche eine vorgegebene Gruppe zur Multiplicatorengruppe besitzen.

Aus der Bemerkung, dass die Klassen als solche durch Multiplication eines jeden Elementes des Schemas $\left(\mathfrak{A}_{\delta}\right)$ mit irgend einem Elemente $B^{\lambda \delta^{j+j}}$ blon eine $j$ malige cyclische Verschiebung erleiden, geht hervor, dass ein System von Elementen, dessen Multiplicatorengruppe genau mit der Klasse (0) übereinstimmen soll, insoferne beschränkt ist, als es nur aus einem Complex von solchen Klassen des Schemas $\left(\mathfrak{H}_{\delta}\right)$ gebildet sein kann, deren Zeiger sich nicht in modulo o geschlossene, arithmetische Progressionen der Form

(II) $i, i+j, i+2 j, \ldots, i+\left(\frac{\delta}{\Theta}-1\right) j \quad(i=0,1,2, \ldots, \overline{\theta-1})$

ordnen lassen, wobei mit $j$ eine bestimmte Zahl der Reihe

$$
1,2, \ldots,(\bar{b}-1)
$$

und mit $\Theta$ der größte gemeinsame Divisor zwischen $j$ und $\delta$ bezeichnet ist. Sonst wäre ja auch das Element $B^{j}$, welches nicht der Klasse (0) angehört, gegen die Annahme Multiplicator.

Umgelkehrt hat jeder Complex von Klassen des Schemas $\left(\mathfrak{A}_{\delta}\right)$, deren Zeiger die angegebene Anordnung nicht besitzen, die Klasse (0) zur Multiplicatorengruppe. Denn würde dann neben den Elementen der Klasse (0) noch das Element $B^{j}$ Multiplicator sein, so müssten sich eben die Klassenzeiger in die angegebene Anordnung (II) bringen lassen.

Man hat mithin, um den Complex $\bar{\Omega}$ und speciell die Anzahl der Systeme, welche eine gegebene Gruppe vom Grade $\frac{n}{\delta}$ zur Multiplicatorengruppe besitzen, zu erhalten, blob aus der Ge- 
sammtheit $\Omega$ aller überhaupt möglichen Klassenverbindungen alle diejenigen Complexe, deren Zeiger sich in die Form (II) setzen lassen, auszuscheiden, was mit Hilfe der Methode des Ausscheidens und Hinzufügens bewerkstelligt werden kann.

Bezeichnet man mit $q_{l}(l=1,2, \ldots, r)$ die verschiedenen Primfactoren von $\delta$, so genügt es für den angegebenen $Z_{w e c k}$ aus dem Complexe

$$
\text { Q, }
$$

welcher $\left(\begin{array}{l}\delta \\ 1\end{array}\right)+\left(\begin{array}{l}\delta \\ 2\end{array}\right)+\cdots+1$, d. h. $2^{\delta}-1$ Klassenverbindungen enthält, alle Complexe

$$
\Omega_{q_{l}}(l=1,2, \ldots, r)
$$

auszuscheiden, welche durch die $\left(2^{\frac{\delta}{q_{l}}}-1\right)$ möglichen Combinationen $\operatorname{der} \frac{\delta}{q_{l}}$ Klassensysteme

$$
\begin{gathered}
i^{\prime}, i^{\prime}+j_{q_{l}}, i^{\prime}+2 j_{q_{l}}, \ldots, i^{\prime}+\left(q_{l}-1\right) j_{q_{l}} \\
\left(i^{\prime}=0,1, \ldots,\left(\frac{o}{q_{l}}-1\right)\right)
\end{gathered}
$$

entstehen, wo für $j_{q_{l}}$ irgend eine der Zahlen

$$
\frac{\delta}{q_{l}}, 2 \frac{\delta}{q_{l}}, \ldots,\left(q_{l}-1\right) \frac{\delta}{q_{l}}
$$

gewählt werden kann, weil für alle diese Zahlen die Reihe (III) modulo os ungeändert bleibt.

In der That muss der größte gemeinsame Divisor $\theta$ von $\delta$ und $j$, der in der Reihe (II) auftritt, mindestens eine der Zahlen $\frac{\delta}{q_{l}}(l=1,2, \ldots, r)$ theilen, da ja $j$ kleiner als $\delta$ ist. Besteht nun die Gleichung

$$
\frac{\delta}{q_{l}}=\Theta \Theta^{\prime}
$$

wo $\Theta^{\prime}$ eine ganze Zahl bedeutet, so kann man die Reihe (II) modulo $\delta$ durch $\Theta^{\prime}$ Reihen (III) ersetzen, indem man dem Zeiger $i^{\prime}$ successive die Werte

$$
i, i+\Theta, i+2 \Theta, \ldots, i+\left(\Theta^{\prime}-1\right) \Theta
$$

ertheilt; denn in beiden Fällen erhält man, abgesehen von dem Addenden $i$ modulo $\delta$ genau diejenigen $q_{l} \theta^{\prime}$ Zahlen des Restsystemes

$$
0,1, \ldots,(\delta-1)
$$

welche durch $\Theta$ theilbar sind. 
Die Reihe (II) kommt also in jedem Complex $\Omega_{q_{l}}(l=1,2, \ldots, r)$, dessen Index ofter in $\delta$, wie in $\theta$ aufgeht, vor und nur in einem solchen Complex; denn innerhalb von $\Omega_{q_{l}}$ treten nur Verbindungen von je $q_{l}$ Klassen auf, so dass die in jeder Combination enthaltene Klassenzahl ein Multiplum von $q_{l}$ sein muss.

Aus dieser Überlegung folgt zugleich, dass der den Complexen

$$
\Omega_{q_{i_{1}}}, \Omega_{q_{i_{2}}}, \ldots, \Omega_{q_{i_{m a}}}
$$

gemeinsame Complex

$$
\Omega_{q_{i_{1}}, \ldots, q_{i_{m}}}
$$

genau durch den Complex $\operatorname{der}\left(2^{\frac{\delta}{q_{i_{1} \cdots q_{i_{m}}}}}-1\right)$ Verbindungen von Reihen (II) dargestellt wird, welche man erhält, indem man alle Werte des laufenden Buchstaben $i$ auf alle möglichen Arten combiniert, während man für $\Theta$ die Zahl $\frac{\grave{o}}{q_{i_{1}} \cdots q_{i_{m}}}$ nimmt.

Setzt man in dem Ausdrucke (I) der Nr. 1 im I. Abschn. an die Stelle von $T$ den Buchstaben $\Omega$, so erhält man den gesuchten Complex $\bar{\Omega}$ durch die Complexe

$$
\Omega, \Omega_{q_{1}}, \Omega_{q_{3}}, \ldots, \Omega_{q_{1}, q_{2}} \ldots \text { etc. }
$$

ausgedrückt. Speciell folgt hieraus das Theorem:

Es gibt innerhalb einer regulären Gruppe vom Grade $n$ genau

$$
\left.2^{\delta}-\sum_{q} 2^{\frac{\delta}{q}}+\sum_{q, q^{\prime}} 2^{\frac{\delta}{q q^{\prime}}}-\cdots 1\right) .
$$

Elementensysteme, deren Multiplicatorengruppe den Grad $\frac{n}{\delta}$ besitzt.

Ganz auf analogem Wege lässt sich sich die Anzahl der Elementensysteme derselben Eigenschaft bestimmen, deren Elementezahl $h$ aber uberdies noch vorgeschrieben ist.

Nach dem Satze (1) muss die Anzahl $k$ ein Vielfaches von $\frac{n}{\delta}$, etwa gleich $u \frac{n}{\delta}$ sein. Es kommen iann überhaupt nur $\left(\begin{array}{l}\hat{o} \\ \mu\end{array}\right)$ Combinationen $C$ im Complexe $\Omega$ in Betracht und es ist leicht einzusehen, dass nur in denjenigen Complexen $\Omega_{q_{i_{1}} \ldots q_{i_{m}}}$, bei denen

1) Man vergl, den Ausdruck von Gauss und Serret für die Anzahl der nach einem Primzahlmodul irreducibelen Functionen ìten Grades. 
die Primfactoren $q_{i_{1}}, \ldots, q_{i_{m}}$ auch Theiler von $\mu$ sind, derartige Combinationen $C$ enthalten sind; und zwar umfasst $\Omega_{q_{i_{1}} \ldots q_{i_{m}}}$ genau

$$
\left(\begin{array}{c}
\frac{\delta}{q_{i_{1}} \cdots q_{i_{m}}} \\
\frac{\mu}{q_{i_{1}} \cdots q_{i_{m}}}
\end{array}\right)
$$

solche Combinationen, woraus sich der folgende Satz ergibt:

Es kommen innerhalb einer regulären Gruppe vom Grade $n$ genau

$$
\left(\begin{array}{l}
\hat{\delta} \\
\mu
\end{array}\right)-\sum_{q}\left(\begin{array}{l}
\frac{\delta}{q} \\
\frac{\mu}{q}
\end{array}\right)+\sum_{q q^{\prime}}\left(\begin{array}{c}
\frac{\delta}{q q^{\prime}} \\
\frac{\mu}{q q^{\prime}}
\end{array}\right)-\cdots
$$

Systeme von je $\mu \frac{n}{\delta}$ Elementen $\vee$ or, deren Multiplicatorengruppe den Grad $\frac{n}{\delta}$ besitzt.

Nr. 10. Über das aus den Elementen einer Gruppe gebildete System von Combinationen zu einer bestimmten Klasse.

Es seien aus den der Gruppe $\mathrm{H}$ angehörigen Elementen

$$
A_{1}, A_{2}, \ldots, A_{k},
$$

welche eine von der Einheit verschiedene Multiplicatorengruppe vom Grade $s$ besitzen mögen, alle Combinationen ohne Wiederholung zur $\delta^{\text {ten }}$ Klasse derart gebildet, dass in jeder Complexion die einzelnen Elemente multiplicativ vereinigt worden sind. Die Gesammtheit der so entstandenen Elemente von $\mathrm{H}$ soll mit

$$
\mathfrak{c}_{\delta}
$$

und die Anzahl, welche angibt, wie oft ein bestimmtes Element $S$ sich im Systeme $\mathfrak{C}_{\delta}$ vorfindet, mit

$$
\mathfrak{c}_{\delta}(S)
$$

bezeichnet werden.

Im Folgenden soll diese Anzahl, auf welche ich durch Untersuchungen über wurzellose Congruenzen geführt wurde, einer eingehenden Betrachtung unterzogen werden. 


\section{§. 1.}

Bezeichnet man mit

\section{$M$}

einen Multiplicator der Reihe (I), so kann man bei der Bildung des Systemes $C_{\delta}$ an Stelle der Elemente (I) die Individuen

$$
M A_{1}, M A_{2}, \ldots, M A_{k}
$$

verwenden, ohne dass hierdurch das Resultat geändert würde, weil beide Reihen bis auf die Aufeinanderfolge der Elemente mit einander übereinstimmen und die Reihenfolge derselben bei der Bildung der Combinationen wegen der Giltigkeit des commutativen Gesetzes belanglos ist.

Es wird mithin die Zahl, welche angibt, wie oft bei der letzteren Bildungsart das Element

$$
M A_{i_{1}} \cdot M A_{i_{2}} \ldots M A_{i_{\delta}}, \text { d. h. } M^{\delta} J
$$

auftritt, einerseits mit dem schon bei der ersten Erzeugungsart eingeführten Symbol

$$
\mathfrak{C}_{\delta}\left(M^{\delta} J\right)
$$

zu. bezeichnen sein. Andererseits ergibt sich unmittelbar, dass das Element $M^{\delta} J$ bei der zweiten Bildungsweise ebensooft auftritt, wie das Individuum $J$ bei der ersten, ein Umstand, der die Gleichung:

$$
\mathbb{C}_{\delta}\left(M^{\delta} J\right)=\mathbb{E}_{\delta}(J)
$$

nach sich zieht.

Man erhält auf diese Weise den Satz:

Im Systeme $\widetilde{C}_{\delta}$ kommen alle Elemente, welche (1) sich voneinander blof um ite Potenzen der gleich oft ror.

Die Gesammtheit der $\delta^{\text {ten }}$ Potenzen der Multiplicatoren bildet aber nach dem Satze (7) der Nr. 6 selbst eine Untergruppe

$$
1, M_{1}, M_{2}, \ldots, M_{t-1}
$$

der Multiplicatorengruppe und es wird das System der versehiedenen Elemente von $\mathfrak{S}_{\delta}$ in Klassen

$$
\left\{\begin{array}{c}
J, M_{1} J, \ldots, M_{t-1} J \\
J^{\prime}, M_{1} J^{\prime}, \ldots, M_{t-1} J^{\prime} \\
\ldots \ldots \ldots \ldots \ldots
\end{array}\right.
$$


zerfallen, deren jede nur Elemente enthält, die in $\widetilde{C}_{\delta}$ gleich oft vorkommen.

Selbstverständlich kann man nach dem Satze (8) der Nr. 6 an Stelle der $\delta^{\text {ten }}$ die $\varepsilon^{\prime \text { ten }}$ Potenzen innerhalb der Multiplicatorengruppe setzen, wenn nur die beiden Zahlen $\delta$ und $\delta^{\prime}$ mit $s$ denselben größten gemeinsamen Theiler besitzen.

Es soll nunmehr der specielle Fall, dass das System (I) eine Abel'sche Gruppe $\mathrm{H}$ vom Grade $n$ ist, also mit seiner Multiplicatorengruppe übereinstimmt, näher untersucht werden.

Unter dieser Annahme enthält der Complex $\mathfrak{S}_{\delta}$ abgesehen von zwei bestimmten Ausuahmen alle Elemente von $\mathrm{H}$, falls rur die Zahl o kleiner als $n$ ist.

Dieser Satz, dessen Richtigkeit tür den Fall $\delta=1$ von selbst einleuchtet, kann mit Hilfe des Schlusses von $\overline{\delta-1}$ auf $\delta$ bewiesen werden $(\hat{0}-1<n-1)$.

Es wird rorausgesetzt, jedes Element $A$ von $H$ lasse sich als das Product

$$
A_{1} \cdot A_{2} \ldots A_{\delta-1}
$$

von $\hat{\delta}-1$ untereinander verschiedenen Elementen darstellen. $\mathrm{Zu}$ zeigen ist, dass dann das Element $A$ auch als das Proủuct von $\delta$ untereinander verschiedenen Factoren aufgefasst werden kann.

Zunächst ist klar, dass eines der Elemente $A_{1}, \ldots, A_{\delta-1}$, z. B. $A_{\delta-1}$ gleich der Einheit angenommen werden darf; sonst hätte man ja nur zum Producte $A_{1} \ldots A_{\delta-1}$ die Einheit als Factor hinzuzufügen, um thatsächlich das Element $A$ als das Product von $\delta$ verschiedenen Elementen dargestellt zu erhalten.

Da ferner die Zahl $\bar{\delta}-1$ nach Annahme den Wert $n-2$ nicht übertrifft, muss es wenigstens zwei von einander verschiedene der Gruppe $\mathrm{H}$ angehörige Elemente

$$
S \text { und } T
$$

geben, welche nicht in der Reihe

$$
1, A_{1}, A_{2}, \ldots, A_{\delta-2}
$$

enthalten sind.

Entsprechend dem Verhalten von $S$ und $T$ theilt sich die Untersuchung in zwei Hauptfälle, bei denen wieder mehrere specielle Annahmen zu unterscheiden sind.

1. Hauptfall: Es existiert wenigstens ein Element $S$, dessen Grad $\sigma$ größer als 2 ist.

Man hat hier, jenachdem das Element $T$ eine Potenz von $S$ ist, oder nicht, zwei Unterfälle zu unterscheiden. 
Im ersteren Falle kann man

$$
T=S^{-i} \quad(0<i<\sigma-1)
$$

setzen und hierbei den Exponenten $i$ so wählen, dass das Element

$$
S^{-i+1}
$$

dem Systeme (IV) angehört. Findet sich ja doch thatsächlich das Element $S^{-1+1}$, d. h. die Einheit in der Reihe (IV) vor.

Indem man nun in der Darstellung (III) das Element $S^{-i+1}$ durch das Product $S . T$ ersetzt, erhält man das Element $A$ als das Product von $\delta$ verschiedenen Factoren.

In dem letzteren Falle hingegen, in welchem das Element $I$ keine Potenz von $S$ ist, darf man von selbst noch die Voraussetzung, dass auch $S$ keine Potenz von $T$ sei, hinzufügen; denn man würde sich sonst, wenn der Grad $\tau$ von $T$ größer als 2 ist, nach Vertauschung von $S$ und $T$ im vorhin erledigten Unterfalle befinden, während für $\tau=2$ die Richtigkeit der gemachten Annahme von selbst einleuchtet.

Man darf ferner voraussetzen, dass sich das Element

\section{$S T$}

nicht in der Reihe (IV) vorfinde; andernfalls hätte man ja bloß für das Element $S T$ das Product der beiden Factoren $S$ und $T$ zu substituieren, um das Element $A$ in der gewünschten Form dargestellt zu erhalten.

Dasselbe gilt aber auch der Reihe nach von den Individuen

$$
S^{2} T ; S^{3} T ; \ldots ; S^{\sigma-1} T ;
$$

denn stets liebe sich das betreffende im Systeme (IV) enthaltene Element $S^{j} T$ durch das Product der beiden verschiedenen, der Annahme nach in der Reihe (IV) nicht vorkommenden Individuen $S$ und $S^{j-1} T$ ersetzen.

Eine analoge Schlussweise lehrt, dass man weiters annehmen darf, dass sich aufer dem Producte $S^{-1} T$ anch das Element

$$
S^{-1} T^{2} \text {, ferner } S^{-1} T^{3} \text { u. s. f. } S^{-1} T^{-1}
$$

in der Reihe (IV) nicht vorfindet.

Hat nun das Element $S T$ einen Grad, welcher größer als 2 ist, so lässt sich in der Darstellung. (III) die Einheit durch die beiden verschiedenen in der Reihe (IV) nicht enthaltenen Factoren $S T$ und $(S T)^{-1}$

ersetzen. 
Aus der Gleichung

$$
(S T)^{2}=1
$$

hingegen folgt, dass in der Darstellung (III) für die Einheit das Product der beiden von einander verschiedenen Elemente $S^{2} T$ und $T$ gesetzt werden kann, vorausgesetzt natürlich, dass das Element $S^{2} T$ nicht unter den Elementen der Reihe (IV) enthalten ist.

Eine Gleichung, wie

$$
A_{1}=S^{2} T
$$

zieht aber die Möglichkeit, für das Element $A_{1}$ das Product $S .(S T)$ zu substituieren, nach sich.

2. Hauptfall: Alle in der Reihe (IV) nicht enthaltenen Elemente der Gruppe $\mathrm{H}$ besitzen den Grad 2.

In diesem Falle kann man, wie vorhin, annehmen, dass außer den Elementen $S$ und $T$ sich auch ihr Product $(S T)$ nicht im Systeme (IV) vorfinde.

Existiert nun innerhalb der Gruppe $\mathrm{H}$ ein Element $B$, dessen Grad $\beta$ größer als 2 ist, so muss die Reihe (IV) neben dem Individuum $B$ der Voraussetzung gemäb auch die von $B$ verschiedene Potenz $B^{-1}$, deren Grad ebenfalls gleich $\beta$ ist, enthalten und man kann in der Darstellung (III) das Product der beiden Elemente

durch das Product

$$
B \text { und } B^{-1}
$$

\section{S.ST.T}

von 3 verschiedenen Factoren ersetzen, so dass auch in diesem Falle das Element $A$ in der verlangten Form ausgedrückt erscheint.

Besitzen hingegen alle Elemente von $\mathrm{H}$ den Grad 2, so ist das eben durchgeführte Verfahren unanwendbar und thatsächlich gilt unter dieser Voraussetzung der zu beweisende Satz in den beiden speciellen Fällen $\delta=2$ und $\delta=n-2$ nicht melr.

Man erkennt nämlich leicht, dass dann das System $\complement_{\delta}$ im Falle, dass $\delta=2$ ist, $z$ war alle anderen Elemente, aber niemals die Einheit enthält; denn die Relation $A_{1} A_{2}=1$ zieht die Gleichheit der beiden Elemente $A_{1}$, und $A_{2}$ nach sich.

Dasselbe gilt auch für das System $\mathbb{C}_{n-2}(n>4)$, weil die beiden Systeme $\mathfrak{C}_{2}$ und $\mathbb{C}_{n-2}$ identisch sind, da das Product aller Elemente von $\mathrm{H}$ gleich der Einheit ist.

Für alle anderen Zahlen $\delta$ jedoch bleibt der Satz bestehen. Ohne Mühe lässt sich derselbe nämlich für $\delta=\$$ nachweisen, während seine allgemeine Giltigkeit unter der Annahme

$$
2<\delta-1<n-3
$$

wieder mit Hilfe des Schlusses ron $\delta-1$ auf $\delta$ dargethan werden kann. 
Der Einfachheit halber mögen im Folgenden die vorhin benutzten Buchstaben ohne Änderung ihrer Bedeutung beibehalten werden.

Zunächst ist klar, dass man die Zahl $\delta-1$ stets ungerade annehmen darf; andernfalls lässt sich das Element $A$ durch das Product

$$
\text { 1. } S . S A_{1} \ldots S A_{\delta-2}
$$

von $\delta$ verschiedenen Factoren darstellen, weil dann die Gleichung

besteht.

$$
S^{\grave{\delta}-1}=1
$$

Sei also die Zahl $(\hat{\delta}-1)$ ungerade.

Man wähle die Basiselemente von $\mathrm{H}$ derart, dass man von den Factoren $A_{1}, A_{2}, \ldots, A_{\delta-2}$ des Elementes $A$ das Individuum $A_{i}$ dann und nur dann als Basiselement aufnimmt, wenn es sich nicht als Product von irgend welchen der vorangegangenen Elemente $A_{\curlywedge}, A_{2}, \ldots, A_{i-1}$ ausdrücken lässt.

Es seien auf diese Weise

$$
B_{1}, B_{2}, \ldots, B_{k}
$$

als die $k$ ersten Basiselemente der Gruppe $\mathrm{H}$ gewonnen worden. Wie die Reihe (V), falls sie nicht selbst schon ein vollständiges Basissystem vorstellt, zu einem solchen ergänzt wird, ist für das Folgende gleichgiltig.

Weil die Anzahl der Individuen $A_{1}, \ldots, A_{\delta-2}$ nach Voraussetzung gerade ist, umfasst die Reihe (IV) nicht alle $2^{k}-1$ aus den Elementen des Systemes (V) gebildeten Combinationen aller Klassen, enthält also mindestens eine derartige Combination

$$
S_{1}
$$

nicht, welche, wie leicht einzusehen ist, als das Product zweier Elemente $A_{l}$ und $A_{m}$ betrachtet werden kann $(l<m)$.

Aufer $S_{1}$ kommen in der Reihe (IV), da die Zahl $\delta-1$ kleiner als $n-3$ angenommen wurde, mindestens noch 3 andere Elemente

$$
S_{2}, S_{3}, S_{4}
$$

von $H$ nicht vor und man darf aus einem schon früher angegebenen Grunde das gleiche Verhalten ron einem jeden Producte je zweier dieser Elemente voraussetzen, also speciell auch von den beiden Producten $S_{2} S_{3}$ und $S_{2} S_{4}$. Diese beiden Elemente sind aber ungleich; es muss daher mindestens eines von ihnen, z. B. $S_{2} S_{8}$ von $S_{1}$ verschieden sein. 
Ersetzt man nun in der Darstellung (III) die beiden Factoren $A_{l}$ und $A_{m}$ durch ihr Product $S_{1}$ und die Einheit durch das Produet $S_{2} \cdot\left(S_{2} S_{3}\right) \cdot S_{3}$, so erhält man die Gleichung $A=S_{1} \cdot S_{2} \cdot\left(S_{2} S_{3}\right) \cdot S_{3} \cdot A_{1} \ldots A_{l-1} A_{l+1} \ldots A_{m-1} A_{m+1} \ldots A_{\delta-2}$ welche das Element $A$ als Product von $\delta$ untereinander verschiedenen Factoren darstellt.

Man ist somit zu dem folgenden Satze gelangt:

Unter den aus den $n$ Elementen einer Abelschen Gruppe H ohne Wiederholung gebildeten Combinationen zur $\delta^{\text {ten }}$ Klasse findet sich jedes Element von $\mathrm{H}$ vor, falls nur o kleiner als $n$ ist. Ausgenommen hiervon sind nur die beiden Fälle, dass alle Elemente von $H$ den Grad 2 besitzen und $\delta=2$ oder $=n-2$ ist, weil dann in den bezeichneten Systemen die Einheit nicht vorkommt.

Aus diesem Satze folgt ohne Weiteres die Übereinstimmung des für die Abel'sche Grappe $\mathrm{H}$ gebildeten Systemes $\left(\mathfrak{B Z}_{\delta}\right)$ mit $\mathrm{H}$ selbst, abgesehen natürlich von den beiden Ausnahmsfällen. Es ergibt sich somit entsprechend dem Satze (7) der Nr. 6 die Gleichung:

$$
\prod_{j=1}^{n} \sum_{j} \bigodot_{\delta}(J)=\left(\begin{array}{l}
n \\
\delta
\end{array}\right) .
$$

Hierbei hat sich das Summenzeichen auf alle Elemente $J$ der ersten verticalen Colonne im Schema $\left(\mathfrak{B}_{\delta}\right)$ zu erstrecken, ferner istr unter $\delta_{j}(j=1, \ldots, s)$ der größte gemeinsame Divisor zwischen $\delta$ und der Gradzahl des $j^{\text {ten }}$ Basiselementes der Gruppe $\mathrm{H}$ zu verstehen, endlich wird mit dem Symbol $\varepsilon_{\delta}(J)$ die Anzahl bezeichnet, welche angibt, wie oft das Element $J$ im Systeme der aus den Elementen von $\mathrm{H}$ gebildeten Combinationen zur $\delta^{\text {teu }}$ Klasse vorkommt.

Nimmt man speciell die Zahl o relativ prim zu $n$ an, so erhält man das folgende Theorem:

Das aus den Elementen von $H$ gebildete System $\mathfrak{C}_{\delta}$ enthält jedes Element ron $\mathrm{H}$

$$
\frac{1}{n}\left(\begin{array}{l}
n \\
\delta
\end{array}\right) \mathrm{mal}
$$

falls die beiden Zahlen o and $n$ theilerfremd sind.

So reducieren sich z. B., falls unter $p$ eine Primzahl verstanden wird, die aus der Einheit und den $p^{\text {ten }}$ Einheitswurzeln gebildeten elementar-symmetrischen Functionen, abgesehen natürlich von dem Producte aller Wurzeln auf ganzzahlige Vielfache der Summe aller Elemente, d. h. auf Null. 


$$
\text { §. } 2 \text {. }
$$

Die in dem vorangehenden Paragraphen durchgeführte Untersuchung der Combinationen zur $\delta^{\text {ten }}$ Klasse, gebildet aus den Elementen einer Gruppe, gestattet eine interessante Specialisierung durch die Einfuihrung der Annahme, dass die der Bildung der Combinationen zu Grunde gelegte Gruppe eine reguläre sei.

Es möge daher in den folgenden Betrachtungen die Gruppe $\mathrm{H}$ von der Form

$$
1, B, B^{2}, \ldots, B^{n-1}
$$

sein.

Das Schema $\left(\mathfrak{B}_{\delta}\right)$ im $\S .1$ geht dann in das Schema $\left(\mathfrak{H}_{\delta}\right)$ der Nr. 6 über and es kommt jedes Element irgend einer Klasse (j) $(j=1, \ldots, s)$ von $\left(\mathfrak{A}_{\delta}\right)$ im Systeme $\mathfrak{C}_{\delta}$ gleich oft vor.

Es lässt sich der Complex $\mathbb{C}_{\delta}$ in doppelter Weise bilden: Einmal, indem man die zu combinierenden Elemente in der Form (I) annimmt, ein andermal, indem man ihnen die Gestalt

$$
1, B^{u}, B^{2 \mu}, \ldots, B^{(n-1) \mu}
$$

ertheilt, wo der Exponent $\mu$ irgend eine zu $n$ theilerfremde Zahl vorstellt. Jeder dem Elemente $B^{j}(0<j<n)$ äquivalenten Combination $B^{\lambda_{1}} B^{\lambda_{2}} \ldots B^{\lambda_{\delta}}$ der ersten Bildungsart entspricht eine dem Elemente $B^{j \mu}$ äquivalente Combination $B^{\lambda_{1}{ }^{\mu}} B^{\lambda_{2, \mu}} \ldots B^{\lambda_{\delta} \mu}$ der zweiten Bildungsart und umgekehrt. Es besteht also die Gleichung.

$$
\widetilde{\complement}_{\delta}\left(B^{j}\right)=\widetilde{\mho}_{\delta}\left(B^{j \mu}\right),
$$

welche wohl zu unterscheiden ist von der Beziehung (II) des $\S .1$.

Nimmt der Exponent $\mu$ successive alle Zahlen, welche relativ prim zu $n$ und untereinander modulo $n$ incongruent sind, an, so durchlauft das Product $j u$ genau die Reihe der modulo $n$ incongruenten Zahlen, welche mit $n$ ein und denselben gröbten gemeinsamen Theiler, nämlich den größten gemeinschaftlichen Divisor zwischen $j$ und $n$ besitzen.

Man erschließt hieraus den Satz:

Alle Potenzen von $B$, deren Exponenten mit $n$ (1) denselben größten gemeinsamen Theiler haben, kommen im Systeme $C_{\delta}$ gleich oft vor.

Besitzen die beiden Zahlen $j$ und $\tau$ den gröbten gemeinschaftlichen Divisor $\Theta$, ist also

$$
=j^{\prime} \theta \text { und } \tau=\tau^{\prime} \theta \text {, }
$$


wo die beiden ganzen Zablen $j^{\prime}$ und $\tau^{\prime}$ theilerfremd sind, so lässt sich stets eine ganze Zahl $x$ derart bestimmen, dass die Summe

$$
j+x \tau
$$

oder $\left(j^{\prime}+x \tau^{\prime}\right) \cdot \Theta$ mit $n$ oder $\frac{n}{\tau} \tau^{\prime}, \Theta$ ebenfalls den größten gemeinsamen Divisor $\theta$ hat, wobei, 'wie im Schema $\left(\mathfrak{H}_{\delta}\right)$, mit $\tau$ der größte gemeinschaftliche Theiler zwischen $\delta$ und $n$ bezeichnet wird. Man braucht zu diesem Zwecke für $x$ nur das Product aller der Primfactoren von $\frac{n}{\tau} \mathrm{zu}$ nehmen, welche nicht in $j^{\prime}$ aufgehen.

Versteht man nun unter $j_{1}$ eine andere von $j$ verschiedene Zahl, welche aber mit $\tau$ denselben größten gemeinsamen Theiler $\Theta$ besitzt, und unter $x_{1}$ die dem $x$ entsprechende Zahl, so erhält man, weil die beiden Summen

$$
j+x \tau \text { und } j_{1}+x_{1} \tau
$$

mit $n$ denselben größten gemeinsamen Theiler besitzen, auf Grund des eben abgeleiteten Theoremes (1) die Beziehung

$$
\mathfrak{\mho}_{\delta}\left(B^{j+x \tau}\right)=\widetilde{S}_{\delta}\left(B^{j_{1}+x_{1} \tau}\right) \text {. }
$$

Die linke, beziehungsweise die rechte Seite dieser Gleichung ist. aber nach dem Satze (1) des $\S .1$ nichts anderes, als $\xi_{\delta}\left(B^{j}\right)$, beziehungsweise $\mathfrak{E}_{\delta}\left(B^{j_{\iota}}\right)$ und es ergibt sich mithin die Gleichung

$$
\mathbb{C}_{\delta}\left(B^{j}\right)=\mathbb{C}_{\delta}\left(B^{j_{1}}\right),
$$

welche in Verbindung mit dem zuletzt benützten Satze das folgende Theorem vermittelt:

Alle Angehörigen derjenigen Klassen $(j)$ des Schemas $\left(\mathfrak{A}_{\delta}\right)$, deren Zeiger $j$ mit $\tau$ denselben größten gemeinschaftlichen Divisor besitzen, finden sich im Systeme $\mathfrak{C}_{\delta}$ gleich oft vor.

Thatsächlich sagt dieses Theorem mehr aus, als der Satz (1) in Verbindung mit dem Satze (1) des vorigen Paragraphen; denn wenn zwei Zahlen mit einer dritten denselben größten gemeinsamen Divisor besitzen, so stehen sie nothwendig auch mit jedem Theiler, aber keineswegs mit jedem Multiplum dieser dritten Zahl in demselben Verhältnis.

Wendet man den Satz (2) auf die Gleichung (VI) des vorausgehenden Paragraphen an, so erhält man die folgende Beziehung:

$$
\frac{n}{\tau} \sum_{t} \aleph_{\delta}\left(B^{t}\right) \varphi\left(\frac{\tau}{t}\right)=\left(\begin{array}{l}
n \\
\delta
\end{array}\right)
$$

in der $t$ alle Theiler von $\tau$ zu durchlaufen hat. 
Für $\tau=1$ gibt diese Gleichung natürlich das dem Satze (3) des §. 1 entsprechende Resultat. Wird hingegen für - eine Primzahl $p$ genommen, so ergibt sich die Relation

$$
\Theta_{\delta}(P)+(p-1) \aleph_{\delta}(N)=\frac{p}{n}\left(\begin{array}{l}
n \\
\delta
\end{array}\right),
$$

in welcher unter $P$ irgend eine $\delta^{\text {to }}$ Potenz, unter $N$ aber ein Element, das keine $\delta^{\text {te }}$ Potenz ist, zu verstehen ist.

\section{§. 3 .}

Es ist klar, dass ein jedes Element $D$ der regulären Abelschen Gruppe $\mathrm{H}$ einen und nur einen Exponenten in Beziehung: auf die Basis $B$ bestimmt, welcher zwischen 0 und $(n-1)$, die Grenzen eingeschlossen, liegt.

Umgekehrt definiert auch jeder solche Exponent ein bestimmtes Element der Gruppe.

Bezeichnet man den durch die Gleichung

$$
S=B^{i} \quad(0 \leqq i \leqq(n-1))
$$

stets bestimmten Exponenten $i$ des Elementes $S$ als den bezüglich der Basis $B$ genommenen Index des Elementes $S$, in Zeichen

$$
i=\text { ind } S \text {, }
$$

so leuchtet ein, dass der Index des Productes ron 2 Elementen modulo $n$ congruent mit der Summe der Indices beider Elemente ist und dass sich vermöge der daraus fließenden Congruenz

$$
\text { ind }\left(A_{1} A_{2} \ldots A_{\delta}\right) \equiv \text { ind } A_{1}+\text { ind } A_{2}+\cdots+\text { ind } A_{\delta}(\bmod n)
$$

jeder vorhin über das System $\mathfrak{E}_{\delta}$ abgeleitete Satz auf dasjenige bezüglich des Moduls $n$ genommene Zahlsystem

$$
\overline{G_{\delta}}
$$

übertragen wird, welches man erhält, wenn man aus den Elementen

$$
0,1, \ldots,(n-1)
$$

alle Combinationen ohne Wiederholung zur $\bar{\delta}^{\text {ten }}$ Klasse bildet und die einzelnen Elemente einer jeden Combination additiv vereinigt, eine Thatsache, deren wahrer Grund in der Nr. 2 des nächsten Abschnittes zu Tage treten wird.

Gremäß dieser Bemerkung nimmt z. B. der Satz (2) des vorigen Paragraphen die folgende Gestalt an: 
Bezeichnet man mit $\tau$ den gröbten gemein(1) samen Divisor zwischen oud $n$, so erzeugt das System $\overline{c_{\delta}}$ modulo $n$ jede Zahl gleich oft, welche mit $\tau$ denselben größten gemeinsamen Divisor besitzt.

Einem jeden'derartigen Satz über den Complex $\overline{{\widetilde{S_{\delta}}}_{\delta}}$ entspricht ein Satz über die Lösungssysteme $x_{1}, x_{2}, \ldots, x_{\delta}$ der Congruenzen der Form

$$
x_{1}+x_{2}+\cdots+x_{\delta} \equiv l \quad(\bmod n),
$$

wobei keine zwei Unbekannten $x_{i}$ und $x_{i^{\prime}}\left(i, i^{\prime}=1,2, \ldots, \delta\right.$; $i$ nicht $=i^{\prime}$ ) einander gleich sein sollen und unter $l$ eine beliebige ganze Zahl zu verstehen ist.

So folgt aus dem Theoreme (1) der Satz:

Wenn die Zahlen $\delta$ und $n$ theilerfremd sind, so besitzt die Congruenz (I) für jeden Wert $l$ genau

$$
(n-1)(n-2) \ldots(n-\delta+1)
$$

Wurzelsysteme.

Umgekehrt kann man natürlich die Congruenz (I) zum Aus. gangspunkt für Untersuchungen über das System $\overline{\mathbb{E}_{\delta}}$ machen. ${ }^{1}$ )

Im Folgenden soll nun der Fall näher untersucht werden, dass der gröbte gemeinsame Theiler $\tau$ zwischen $\delta$ und $n$ eine Primzahl $p$ sei.

1) Von dem Systeme der $n$ Congruenzen

$$
a_{1} x_{1}+a_{2} x_{2}+\cdots+a_{\delta} x_{\delta} \equiv l \quad(\bmod n) \quad(l=0,1, \ldots, \overline{n-1})
$$

gelten nämlich, ob man nun die Gleichheit zweier Unbekannten zulässt oder nicht, allgemein die beiden folgenden Sätze:

1. Alle Congruenzen, deren rechte Seiten mit $n$ denselben größten gemeinsamen Theiler besitzen, haben die gleiche Anzahl von Lösungssystemen $x_{1}, x_{2}, \ldots, x_{\delta}$.

2. Alle Congruenzen, deren rechte Seiten sich modulo $n$ nur uin ganzzahlige Vielfache der Summe

$$
a_{1}+a_{2}+\cdots+a_{\delta}
$$

unterscheiden, besitzen die gleiche Anzahl von Lösungssystemen $x_{1}, x_{2}, \ldots, x_{\delta}$.

Bezeichnet man nämlich eine Congruenz (II), deren rechte Seite mit irgend einer Zahl $k$ congruent ist, der Kürze halber als Congruenz $(k)$, so wird je einem Lösungssystem $x_{1}, x_{2}, \ldots, x_{\delta}$ einer Congruenz $(l)$, wie man durch Multiplication derselben mit $\mu$ erkennt, sich je ein Lösungssystem $\mu x_{1}, \mu x_{2}, \ldots, \mu x_{\delta}$ der Congruenz $(\mu, \zeta)$ zuordnen und umgekehrt, falls nur die beiden Zahlen $\mu$ und $n$ theilerfremd sind.

Das Gleiche gilt auch ron den beiden den Congruenzen $(l)$ und $\left(l+p \overline{a_{1}+a_{2}+\cdots+a_{\delta}}\right)$ angehörigen Lösungssystemen

$$
x_{1}, x_{2}, \ldots, x_{\delta} \text { und } x_{1}+\rho, x_{2}+\rho, \ldots, x_{\delta}+p \text {. }
$$


Dann enthält nach dem Satze (1) das System $\overline{\mathbb{S}_{\delta}}$ jede Zahl

$$
j p\left(j=0,1, \ldots,\left(\frac{n}{p}-1\right)\right)
$$

modulo $n$ genan $\mathfrak{S}_{\delta}(P)$ mal, d. h. so oft, als in dem Systeme $\widetilde{C}_{\delta}$ eine $\delta^{\text {te }}$ Potenz $P$ der Abel'schen Gruppe $H$ vorkommt, jede andere Zahl

$$
j p+r\left(r=1,2, \ldots, \overline{p-1} ; j=0,1, \ldots,\left(\frac{n}{p}-1\right)\right)
$$

bingegen $\mathfrak{C}_{\partial}(N)$ mal, wo mit $N$ irgend ein Element von $H$ bezeichnet wird, das keine s $^{\text {te }}$ Potenz ist.

Bezeichnet man also mit $\rho$ eine $p^{\text {te }}$ Einheitswurzel und setzt. man in der Summe

$$
\sum_{i_{1}, \ldots, i_{\delta}}^{i_{1}+i_{2}+\cdots+i_{\delta}}
$$

welche sich auf alle aus den Elementen $0,1, \ldots, \overline{n-1}$ ohne Wiederholung gebildeten Combinationen $i_{1}, i_{2}, \ldots, i_{\delta} \mathrm{zu}$ erstrecken hat, für einen jeden Exponenten $i_{1}+i_{2}+\cdots+i_{\delta}$ seinen kleinsten nicht negativen Rest modulo $n$ genommen, so erhält man unter Berücksichtigung der Bezichung

$$
\rho^{\mu p}=1 \quad(\mu=0,1,2, \ldots)
$$

die Gleichung

$$
\sum_{i_{1}, \ldots, i_{\delta}} p^{i_{1}+i_{2}+\cdots+i_{\delta}}=\mathfrak{\Xi}_{\delta}(P) \frac{n}{p}+1-\Xi_{\delta}(N) \sum_{j=0}^{\frac{n}{p}-1} \sum_{r=1}^{p-1} p^{j p+r},
$$

deren rechte Seite den Wert

besitzt.

$$
\frac{n}{p}\left[c_{\delta}(P)-c_{\delta}(N)\right]
$$

Der Wert der linken Seite der Gleichung (III) kann hingegen. auf folgendem Wege ermittelt werden:

Bedeutet $x$ eine variable Gröfíe und werden in dem Producte.

$$
(x-1)(x-p)\left(x-p^{2}\right) \ldots\left(x-p^{n-1}\right)
$$

je $p$ unmittelbar aufeinanderfolgende Factoren zu einem einzigen Factor $\left(x^{p}-1\right)$ vereinigt, so ergibt sich die folgende Identität nach $x$ :

$$
\prod_{i=0}^{i=n-1}\left(x-\rho^{i}\right)=\left(x^{p}-1\right)^{\frac{n}{p}} \text {. }
$$


Entwickelt man beide Seiten derselben nach fallenden Potenzen der Variabelen $x$ und vergleicht man hierauf die Coefficienten der Potenz $x^{n-\delta}$ beiderseits, so erhält man die Relation

$$
(-1)^{\delta} \sum_{i_{1}, \ldots, i_{\delta}} p^{i_{1}+i_{2}+\cdots+i_{\delta}}=(-1)^{\frac{\delta}{p}}\left(\begin{array}{c}
\frac{n}{p} \\
\frac{\delta}{p}
\end{array}\right),
$$

welche in Verbindung mit der Gleichung (III) die Beziehung

$$
\varsigma_{\delta}(P)-\varsigma_{\delta}(N)=\eta \frac{p}{n}\left(\begin{array}{c}
\frac{n}{p} \\
\frac{\delta}{p}
\end{array}\right)
$$

liefert, wo das Zeichen $\eta$ die negative oder positive Einheit bedeutet, je nachdem der Fall $p=2, \delta=2(2 \mu+1)$ eintritt, oder nicht.

Das Resultat (IV) gibt im Zusammenhalte mit der Gleichung: (III) des vorigen Paragraphen den folgenden Satz:

Das System $\mathbb{C}_{\delta}$, beziehungsweise $\overline{\mathbb{C}_{\delta}}$, erzeugt jede $\delta^{\text {te }}$ Potenz, beziehungsweise jedes Multiplum von $p$

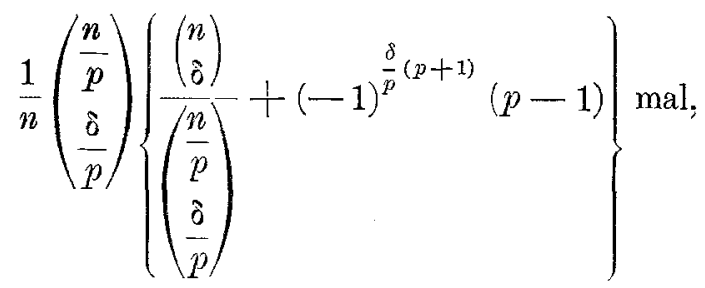
(2) jedes andere Element, beziehungsweise jede
andere Zahl aber

$$
\frac{1}{n}\left(\begin{array}{l}
\frac{n}{p} \\
\frac{\delta}{p}
\end{array}\right)\left\{\frac{\left(\begin{array}{l}
n \\
\delta
\end{array}\right)}{\left(\begin{array}{c}
\frac{n}{p} \\
\frac{\delta}{p}
\end{array}\right)}-(-1)^{\frac{\delta}{p}(p+1)}\right\} \mathrm{mal},
$$

falls der gröbte gemeinsame Theiler $p z w i s c h e n$ $s$ und $n$ eine Primzahl ist.

Aus diesem Satze folgt ohne Weiteres die Bestimmung der Anzahl der Lösungssysteme $x_{1}, x_{2}, \ldots, x_{\delta}$ der Congruenz (I) in dem 
Falle, dass die Zahlen $\delta$ und $n$ die Primzahl $p$ zum größten gemeinsamen Theiler besitzen.

Wenn der Grad $n$ der regulären Gruppe $H$ gerade ist, so existiert ein und nur ein Element $B^{\frac{n}{2}}$, das auf das Quadrat erhoben die Einheit zum Resultat ergibt. Dieses Element wird daher passend als negative Einheit der Gruppe $\mathrm{H}$ mit -1 bezeichnet werden. Element

Für das Product aller Elemente von $H$ ergibt sich das

$$
E=B^{\frac{n(n-1)}{2}}
$$

d. h. +1 oder -1 , je nachdem $n$ ungerade oder gerade ist. Es ordnet sich mithin einer jeden Combination

$$
B^{i_{1}} B^{i_{2}} \ldots B^{i_{\delta}}
$$

des Systemes $\mathfrak{C}_{f}$ eine Combination

$$
B B_{1}^{i_{1}} B i_{2}^{\prime} \ldots B^{i_{n-\delta}^{\prime}}
$$

des Systemes $\mathbb{E}_{n-\delta}$, welche die iubrigen der Elemente $1, B, \ldots, B^{n-1}$ umfasst, derart zu, dass die Gleichung

$$
\left(B^{i_{1}} \ldots B^{i_{\delta}}\right)\left(B^{i_{1}^{\prime}} \ldots B^{i_{n}^{\prime}-\delta}\right)=E
$$

besteht. Erzeugt demnach die Combination $B^{i_{1}} \ldots B^{i_{\delta}}$ das Element $E B^{\alpha}$, so erzeugt die ergänzende Combination $B^{i_{1}^{\prime}} \ldots B^{i_{n}^{\prime}-\delta}$ das Element $B^{n-\alpha}$ and umgekehrt; es besteht somit die Relation $\mathbb{E}_{\delta}\left(E B^{i}\right)=\mathbb{C}_{n-\delta}\left(B^{n-i}\right)$, aus welcher nach dem Satze (1) des vorigen Paragraphen die Gleichung:

folgt.

$$
\mathfrak{\subseteq}_{\delta}\left(E B^{i}\right)=\mathfrak{\complement}_{n-\delta}\left(B^{i}\right)
$$

Es ergibt sich daher das Theorem:

Ist die Zahl $n$ ungerade, so stimmt das System $\mathbb{S}_{n-\delta}$ mit dem System $\widetilde{C}_{\delta}$ vollständig über(3) ein. Ist jedoch die Zahl $n$ gerade, so hat man alle Elemente von $c_{\delta}$ mit der negativen Einheit zu multiplicieren, um diejenigen des Systemes $\mathfrak{C}_{n-\delta}$ zu erhalten. 
§. 4.

Im Folgenden soll eine Methode angegeben werden, welche gestattet, zu jedem numerisch vorgegebenen Wertepaar is und : die Anzahl $\widetilde{\Xi}_{\delta}\left(B^{j}\right)$ als Function des Grades $n$ auszudrücken, sobald die Zahl $n$ nur eine bestimmte Grenze überschreitet.

Einerseits wird eine Combination des Systemes $\varepsilon_{\delta}$, welche sich aus $k_{0}$ Elementen der Klasse (0), aus $k_{1}$ Elementen der Klasse (1), .., aus $k_{\tau-1}$ Elementen der Klasse $(\tau-1)$ des Schemas $\left(\mathfrak{H}_{\delta}\right)$ in Nr. 6 zusammensetzt, nur dann der Klasse $(j)$ angehören, wenn die Bedingung:

$$
\begin{gathered}
k_{0} .0+k_{1} \cdot 1+\cdots+k_{\tau-1}(\tau-1) \equiv j(\bmod \tau) \\
\left(k_{i} \geq 0 ; i=0,1, \ldots, \tau-1\right)
\end{gathered}
$$

erfüllt ist, zu welcher von selbst noch die beiden folgenden Nebenbedingungen :

$$
k_{0}+k_{1}+\cdots k_{\tau-1}=\dot{0}
$$

und

$$
k_{0} ; k_{1} ; \ldots ; k_{\tau-1} \leq \frac{n}{\tau}
$$

treten.

Hat man andererseits umgekehrt irgend ein System ganzer Zahlen $k_{0}, k_{1}, \ldots, k_{\tau-1}$ gefunden, welches die Forderungen (I), (II) und (III) erfüllt und vereinigt man multiplicativ $k_{0}$ beliebige Elemente der Klasse (0), $k_{1}$ beliebige Elemente der Klasse (1)..., $k_{x \rightarrow 1}$ beliebige Elemente der Klasse $(\tau-1)$ des Schemas $\left(\mathfrak{A}_{\delta}\right) \mathrm{zu}$ einer Combination, so gehört dieselbe der Klasse $(j)$ an.

Einer jeden Darstellung der Zahl $j$ modulo $\tau$ durch die ohne Rücksicht auf den Modul $\tau$ zu nehmenden ganzen Zahlen $k_{0}, k_{1}, \ldots, k_{z-1}$, wie sie die Bedingung (I) mit den Nebenbedingungen (II) und (III) vorschreibt, entsprechen somit

$$
\left(\begin{array}{c}
\frac{n}{\tau} \\
k_{0}
\end{array}\right)\left(\begin{array}{c}
\frac{n}{\tau} \\
k_{1}
\end{array}\right) \ldots\left(\begin{array}{c}
\frac{n}{\tau} \\
k_{\tau-1}
\end{array}\right)
$$

verschiedene Combinationen des Systemes $\mathfrak{C}_{\delta}$, welche alle der Klasse $(j)$ angehören; und jede deratige Combination wird auf die geschilderte Art erhalten.

Da aber durch das System $\mathfrak{C}_{\delta}$ alle Elemente einer Klasse nach dem Satze (1) des §. 1 gleich oft erzeugt werden, erhält man die gesuchte Anzahl jener Combinationen des Systemes $\vec{E}_{\delta}$, 
welche mit einem vorgegebenen Element $B^{j}$ übereinstimmen, durch die Gleichung:

$$
\mathfrak{E}_{\delta}\left(B^{j}\right)=\frac{\Sigma}{n} \sum_{k_{0}, \ldots, k_{\tau}-1}\left(\begin{array}{c}
\frac{n}{\tau} \\
k_{0}
\end{array}\right)\left(\begin{array}{c}
\frac{n}{\tau} \\
k_{1}
\end{array}\right) \ldots\left(\begin{array}{c}
\frac{n}{\tau} \\
k_{\tau-1}
\end{array}\right)
$$

bestimmt, in welcher sich die Summe über alle den Bedingungen (I), (II) und (III) genügenden Systeme $k_{0}, k_{1}, \ldots, k_{\tau-1}$ zu erstrecken hat.

Es ist zu bemerken, dass die Bedingung (III) tür alle Zahlen

$$
n \geq \delta \tau
$$

erfüllt ist, und dass die Bedingungen (I) und (II) unabhängig von dem Grade $n$ sind, sobald die beiden Zahlen $\delta$ und $\tau$ als gegeben betrachtet werden. Bestimmt man demnach für ein numerisch gegebenes Wertepaar $\delta$ und $\tau$ alle Lösungssysteme $k_{0}, \ldots, k_{\tau-1}$, so erhält man auf Grund der Gleichung (IV) die gesuchte Anzahl als Function des Grades $n$ ausgedrückt, vorausgesetzt, dass für $n$ ein solches Multiplum von $\tau$ genommen wird, welches mit $\delta$ den größten gemeinsamen Theiler $\tau$ besitzt und nicht kleiner als $\delta \tau$ ist.

Bei einer wirklichen Berechnung der Anzahlen $C_{\delta}\left(B^{j}\right)$ $(j=0,1, \ldots, \overline{\tau-1})$ lässt sich einer der zu suchenden Werte der Relation (II) des \&. 2 entnehmen. Ferner kann man sich auf Zahlen $j$ beschränken, welche Theiler von $\tau$ sind; es folgt dies aus dem Satze (2) l. c., der übrigens auch im Vorstehenden seine Bestätigung findet.

Maltipliciert man nämlich die Relation (I) mit einer zu $\tau$ theilerfremden Zahl $\mu$, so erhält man wieder eine Congruenz derselben Form

$$
k_{0}^{\prime} \cdot 0+k_{1}^{\prime} \cdot 1+\cdots+k_{\tau-1}^{\prime}(\tau-1) \equiv \mu j(\bmod \tau),
$$

weil die Reihe

$$
0 . \mu, 1 . \mu, \ldots, \overline{\tau-1} \cdot \mu
$$

ebenfalls ein vollständiges Restsystem modulo $\tau$ darstellt.

Aus einem jeden Lösungssystem $k_{0}, \ldots, k_{\tau-1}$ der Congruenz (I) lässt sich also ein Lösungssystem $k_{0}^{\prime}, \ldots, k_{\tau-1}^{\prime}$ der Congruenz $\left(I^{\prime}\right)$ herleiten, das einfach durch Änderung der Aufeinanderfolge der Werte $k_{0}, \ldots, k_{\tau-1}$ erhalten wird. Da auch das Umgekehrte gilt, folgt, dass die Lösungssysteme der beiden Congraenzen (I) und ( $\left.I^{\prime}\right)$ bis auf die Reihentolge ihrer Elemente mit einander übereinstimmen. Die Aufeinanderfolge der Werte $k_{0}, \ldots, k_{t-1}$ ist aber auf die Bedingungen (II) und (III) und auf die Gleichung (IV) ohne Einfluss. Die Anzahl $\mathfrak{C}_{\delta}\left(B^{j}\right)$ ist also gleich der Zahl $\mathbb{E}_{\delta}\left(B^{j \mu}\right)$. 
Als Beispiele mögen die Fälle $\delta=2,3$ und 4 dienen, wobei noch überdies $\tau=\delta, n=K \delta$ und die ganze Zahl $K>\hat{o}$ vorausgesetzt wird.

Das System $\mathfrak{C}_{2}$ erzeugt jedes Quadrat $\frac{n-2}{2}$ mal, jedes andere Element $\frac{n}{2}$ mal.

Das System $\tilde{S}_{3}$ erzeugt jeden Cubus $\left(\frac{n(n-3)}{6}+1\right)$ mal, jedes andere Element $\frac{n(n-3)}{6}$ mal.

Das System $\mathfrak{C}_{4}$ erzeugt jedes Biquadrat $\frac{n-4}{4}\left(\frac{n(n-2)}{6}+1\right)$ mal, jedes Element der Klasse (1) und (3) des Schemas $\left(\mathfrak{A}_{4}\right)$ (3)

$$
\begin{aligned}
& \frac{n(n-2)(n-4)}{1.2 .3 .4} \text { mal, jeden Angehörigen der Klasse } \\
& \frac{n}{4}\left(\frac{n-2)}{6}+1\right) \text { mal. }
\end{aligned}
$$

Der letzte Satz des vorigen Paragraphen bestimmt hierzu von selbst die betreffenden Anzahlen für die Systeme $\mathbb{C}_{n-2}, \widetilde{E}_{n-3}$ und $\widetilde{C}_{n-4}$. Die Sätze (1) und (2) geben natürlich zugleich Beispiele für das allgemeine Theorem (2) I. c.

Nachdem im Vorstehenden die Anzahl $\mathbb{E}_{\delta}\left(B^{j}\right)$ einer eingehenden Betrachtung unterzogen worden ist, mögen hier noch einige Bemerkungen über die mit ihr verwandte Anzahl

$$
\vartheta(j, p)
$$

Platz finden, welche angibt, wie viele der dem Elemente $B^{j}$ äquivalenten Combinationen des Systemes $\mathcal{C}_{\delta}$ die Bedingung erfüllen, dass genau $c$ der sie constituierenden Gruppenelemente der Klasse (p) des Schemas $\left(\mathfrak{H}_{\delta}\right)$ angehören.

Wie schon im $\S .1$ ausführlich auseinandergesetzt wurde, kann man die Bildung des Systemes $\widetilde{C}_{\delta}$ in doppelter Weise bewerkstelligen : elemente

Einmal unter Zugrundelegung der ungeänderten Gruppen-

$$
1, B, \ldots, B^{n-1}
$$

ein andermal mit Hilfe der Reihe

$$
S, S B, \ldots, S B^{n-1}
$$

wo unter $S$ ein beliebiges Gruppenelement verstanden werden kann. Setzt man also für $S$ speciell die $\delta^{\text {ts }}$ Potenz $B^{\delta}$, so erhält man, 
weil alle Elemente einer und derselben Klasse sich von einander nur um ${ }^{\text {te }}$ Potenzen multiplicativ unterscheiden, die Gleichung

$$
\vartheta(j, \rho)=\vartheta(j+\delta . \delta \mu, \rho)
$$

für $u=0,1,2, \ldots$, welche, sobald man für $\frac{n}{\tau}$ eine mit $\tau$ theilerfremde Zahl annimmt, in die der Beziehung (II) des \&. 1. entsprechende Relation

$$
\vartheta(j, p)=\vartheta(j+\mu \delta, p) \quad(\mu=0,1,2, \ldots)
$$

übergeht.

Nun lässt sich die Anzahl derjenigen Combinationen des Systemes $C_{\delta}$, welche $c$ bestimmte und sonst keine der Klasse ( 0 )

angehörige Elemente enthalten, leicht angeben; sie beträgt $\left(\begin{array}{l}n-\frac{n}{\tau} \\ \delta-c\end{array}\right)$, so dass sich für die Gesammtzahl der Combinationen mit genau $c$ beliebigen, der Klasse ( $\rho$ ) angehörigen Elementen der Ausdruck $\left(\begin{array}{c}\frac{n}{\tau} \\ c\end{array}\right)\left(\begin{array}{c}\frac{n}{\tau}(\tau-1) \\ \delta-c\end{array}\right)$

die Gleichung

$$
\frac{n}{\tau} \sum_{j=0}^{\tau-1} \vartheta(j, \rho)=\left(\begin{array}{c}
\frac{n}{\tau} \\
c
\end{array}\right)\left(\begin{array}{c}
\frac{n}{\tau}(\tau-1) \\
\delta-c
\end{array}\right)
$$

folgt. Man vergleiche mit ihr die Relation (VI) des \&. 1.

Ferner leuchtet ein, dass die Überlegungen, welche zur Gleichung (IV) geführt haben, fast wörtlich auf den vorliegenden Fall übertragen werden können. Man erhält auf diese Weise mit Hilfe der Relation (V) die Beziehung:

(VI) $=\frac{\tau}{n}\left(\begin{array}{c}n \\ \tau \\ c\end{array}\right)_{k_{0}, \ldots, k_{\varrho-1}, k_{\varrho+1}, \ldots, k_{\tau-1}}\left(\begin{array}{c}\frac{n}{\tau} \\ k_{0}\end{array}\right) \ldots\left(\begin{array}{c}\frac{n}{\tau} \\ k_{\varrho-1}\end{array}\right)\left(\begin{array}{c}\frac{n}{\tau} \\ k_{\varrho+1}\end{array}\right) \ldots\left(\begin{array}{c}\frac{n}{\tau} \\ k_{\tau-1}\end{array}\right)$,

wo sich die Summe auf alle jene ganzzahligen Wertesysteme $k_{0}, \ldots, k_{\varrho-1}, k_{\varrho+1}, \ldots, k_{\tau-1}$ zu erstrecken hat, welche nach Hinzufügung des constanten Wertes $k_{\varrho}$ gleich $c$ die Bedingungen (I), (II) und (III) befriedigen. 
Durch Addition der beiden Beziehungen (I) und (II) erkennt man sofort, dass sich aus jedem den Forderungen (I) und (II) genügenden Zahlsystem

$$
k_{0}, \ldots, k_{\varrho-1}, k_{\varrho}=c, k_{\varrho+1}, \ldots, k_{\tau-1}
$$

eine dieselben Bedingungen erfüllende Wertereihe

$k_{0}^{\prime}=k_{\tau-1}, k_{1}^{\prime}=k_{0}, \ldots, k_{\varrho}^{\prime}=k_{\varrho-1}, k_{\varrho+1}^{\prime}=c, k_{\varrho+2}^{\prime}=k_{\varrho+1}, \ldots, k_{\tau-1}^{\prime}=k_{\tau-2}$ ergibt, und es ist klar, dass auch die Umkehrung hiervon gilt. ${ }^{1}$ )

1) Bezeichnet man die Congruenz (I) kurz als Congruenz (j), so ordnet sich jedem ohne Rücksicht auf den Modul $\tau$ zu nehmenden Lösungssystem

$$
k_{0}, \ldots, k_{\varrho}, \ldots, k_{\tau-1}
$$

der Congruenz (j), welches die Bedingung (II) und noch überdies die Forderung erfüllt, ein Liösungssystem

$$
k_{\varrho} \geqq c
$$

$$
\begin{aligned}
k_{0}^{\prime}=k_{0}, \ldots, k_{\varrho-1}^{\prime}=k_{\varrho-1}, k_{\varrho}^{\prime}=k_{\varrho}-c, k_{\varrho+1}^{\prime}=k_{\varrho+1}+c \\
k_{\varrho+2}^{\prime}=k_{\varrho+2}, \ldots, k_{\tau-1}^{\prime}=k_{\tau-1}
\end{aligned}
$$

der Congruenz $(j-+-c)$ zu, welches zwar ebenfalls der Bedingung (II), jedoch außerdem der Forderung

$$
h_{\varrho+1}^{\prime} \geqq c
$$

Genüge leistet. Da auch die Umkehrung hiervon gilt, ist die Anzahl der beiden Lösungssysteme die gleiche.

Für die Forderung $k_{\varrho+1}^{\prime} \geqq c$ kann aber nach der i. T. gegebenen Überlegung auch die Bedingung $k_{\varrho}^{r} \geqq c$ gesetzt werden, ohne dass die betreffende Anzahl geändert wird.

Man erhält also den Satz:

Die Zahl der ohne Rücksicht auf den Modul $\tau$ genommenen den Congruenzen $(j)$, bezw. $(j+c)$, bezw. $(j+2 c)$ u. s. f. genügenden Wertsysteme $k_{0}, \ldots, k_{\tau-1}$, welche die Bedingung (II) nebst der Forderung $k_{\varrho} \geqq c$ erfüllen, ist constant.

Nimmt man speciell den Wert $c$ relativ prim zu $\tau$ an, so stellt die Reihe $j, j+c, \ldots, j+\overline{\tau-1} c$ ein vollständiges Restsystem modulo $\tau$ dar und die be* wusste Anzahl wird in diesem Falle gleich

$$
\frac{\mathfrak{n}^{(c)}}{\tau}
$$

wenn die Anzahl der der Gleichung (II) nebst der Bedingung $k_{o} \geqq c$ genügenden nicht negatizen ganzzahligen Wertesysteme $k_{0}, \ldots, k_{\tau-1}$ mit $\mathfrak{n}^{(c)}{ }^{0}$ bezeichnet wird.

Man erkennt also, dass die Zahl $n^{(c)}$ durch $\tau$ theilbar ist; und weil die Differenz $\left(\mathfrak{n}^{(1)}-\mathfrak{n}^{(2)}\right)$ die Anzahl der Darstellungen von $(\delta-1)$ durch $(\tau-1)$ nicht negative ganzzahlige Addenden angibt, erhält man den Satz:

Bezeichnetmanmit $\mu$ einebeliebige, mit abereine ungerade, positive ganze Zahl, so ist die Anzahl der Darstellungen von $\mu \tau-1$ als Summeron $\tau-1$ nicht negativen Zahlen ein MuItiplum von. 
Eine cyclische Vertauschung der Werte $k_{0}, k_{1}, \ldots, k_{\tau-1}$ lässt aber die rechte Seite der Gleichung (VI) ungeändert; man erhält mithin die Beziehung:

$$
\vartheta(j, p)=\vartheta(j, p+1)
$$

aus welcher sich unter Zuziehung der Gleichung (V) der folgende Satz ergibt:

Die Anzahl derjenigen dem Elemente $S$ äquivalenten Combinationen des Systemes $\mathbb{C}_{\delta}$, deren constituierende $\delta$ Gruppenelemente genau $c$ der Klasse (p) angehörige Individuen enthalten, (4) bleibt sowohl für jedes derselben Klasse angehörige Element $S$, als auch für jede Wahl der Klasse (p) ungeändert, vorausgesetzt, dass jeder Primfactor von $\tau$ in derselben Potenz in $\tau$, wie in $n$ a ufgeht.

Zum Schlusse mag noch ohne auf diesen Gegenstand näher einzugehen darauf hingewiesen werden, dass sich aus der Gleichung (IV) auf Grund des Theoremes (2) des vorigen Paragraphen zwei Relationen zwischen Binomialcoefficienten ergeben, je nachdem $j=0$ angenommen wird oder nicht. Setzt man das Zahlenpaar $\delta$ and $\tau$ als gegeben voraus, so werden beide Seiten einer jeden dieser Gleichurgen nach Kürzung mit $\frac{1}{n}$ ganze rationale Functionen von $n$. Beide Beziehungen müssen also, weil sie für unendlich viele ganze Zahlen $n$ gelten, Identitäten nach $n$ sein; und durch Gleichsetzen der Coefficienten der gleichhohen Potenzen von $u$ auf beiden Seiten werden sich Beziehungen zwischen den Lösungssystemen $k_{0}, \ldots, k_{\tau-1}$ ergeben.

\section{§.}

Mit den vorstehenden Untersuchungen über die Combinationen $\mathfrak{C}_{\delta}$ ohne Wiederholung sind zugleich die Variationen $V_{\delta}$ ohne Wiederholung erledigt; denn die letzteren werden durch Permutation der ersteren erhalten, so dass das System $V_{\delta}$ aus dem Systeme $\mathbb{C}_{\delta}$ dadurch hervorgeht, dass man das letztere $\delta !$ mal anschreibt.

Was das System der Combinationen mit Wiederholung betrifft, hat man die Untersuchung analog, wie vorhin, zu führen, um zu ähnlichen Resultaten zu gelangen.

Die Variationen mit Wiederholung endlich geben zu keinen weiteren Betrachtungen Anlass, indem durch sie jedes Element 
der Gruppe gleich oft erzeugt wird. Man erhält nämlich die Variationen zur $\delta^{\text {ten }}$ Klasse aus denen zur $(\delta-1)^{\text {ten }}$ dadurch, dass man jede Complexion der letzteren mit allen Gruppenelementen verbindet, also durch die Gruppe selbst ersetzt.

\section{Abschnitt. Über einige in der Zahlentheorie auftretende Abel'sche Gruppen.}

\section{Nr. 1. Die $k$ zahligen Zahlsysteme.}

Der Complex von irgend welchen $k$ ganzen Zahlen

$$
a_{1}, a_{2}, \ldots, a_{k}
$$

möge mit $S_{k}\left(a_{i}\right)$ bezeichnet werden. Aus je zwei Zahlsystemen $S_{k}\left(a_{i}\right)$ und $S_{k}\left(a_{i}^{\prime}\right)$ lässt sich entsprechend der additiven, bezw. multiplicativen Verbindung der einzelnen Elemente $a_{i}$ und $a_{i}^{\prime}$ $(i=1,2, \ldots, k)$ ein neues System $S_{k}\left(a_{i}+a_{i}^{\prime}\right)$, bezw. $S_{k}\left(a_{i} a_{i}^{\prime}\right)$. herleiten, was durch die Gleichungen

und

$$
S_{k}\left(a_{i}\right) \pm S_{k}\left(a_{i}^{\prime}\right)=S_{k}\left(a_{i} \pm a_{i}^{\prime}\right)
$$

$$
S_{k}\left(a_{i}\right) S_{k}\left(a_{i}^{\prime}\right)=S_{k}\left(a_{i} a_{i}^{\prime}\right)
$$

ausgedrückt werden möge. Aus diesen Beziehungen folgt von selbst nicht nur die Giltigkeit des commutativen und associativen, sondern auch die des distributiven Gesetzes:

$$
S_{k}\left(b_{i}\right)\left[S_{k}\left(a_{i}\right) \pm S_{k}\left(a_{i}^{\prime}\right)\right]=S_{k}\left(b_{i}\right) S_{k}\left(a_{i}\right) \pm S_{k}\left(b_{i}\right) S_{k}\left(a_{i}^{\prime}\right)
$$

Nach diesen Festsetzungen bedarf es keiner weiteren Auseinandersetzung, was man unter Ausdrücken, wie die Summe oder das Product zweier Systeme u. s. f. zu verstehen haben wird. Weiters leuchtet ein, dass falls die Zahl $d_{i}$ der gröbte gemeinsame Theiler zwischen $a_{i}$ und $a_{i}^{\prime}(i=1,2, \ldots, k)$ ist, das System $S_{k}\left(d_{i}\right)$ als der größte gemeinsame Divisor der beiden Complexe $S_{k}\left(a_{i}\right)$ and $S_{k}\left(a_{i}^{\prime}\right)$ zu bezeichnen sein wird. Nach dieser Definition existiert nur dann ein größter gemeinsamer Theiler zweier Systeme $S_{k}\left(a_{i}\right)$ und $S_{k}\left(a_{i}^{\prime}\right)$, wenn keine zwei Zahlen $a_{i}$ and $a_{i}^{\prime}$ mit dem nämlichen Index gleichzeitig Null sind.

Zwei Systeme heilien theilerfremd, wenn sie das System $S_{k}(1)$ zum größoten gemeinsamen Divisor besitzen.

Der größte gemeinsame Theiler zweier Systeme kann auch hier durch einen dem gewöhnlichen entsprechenden Algorithmus 
bestimmt werden; nur ist zu beachten, dass einige Elemente der bei der Durchführung der Rechnung als Reste auftretenden Zahlsysteme auch Null werden können, so dass schließlich für den gesuchten größten gemeinsamen Theiler der größte gemeinsame Divisor zweier aufeinanderfolgender Restsysteme, $d$. h. bei genügend weit fortgesetztem Verfahren die Summe derselben zu nehmen ist.

Es ist klar, dass alle Gesetze der Theilbarkeit erhalten bleiben. So gilt z. B. der Satz: Ist ein Product zweier Systeme durch ein drittes theilbar, welches mit dem einen Factor theilerfremd ist, so muss der andere Factor durch dasselbe theilbar sein. Hieraus folgt die Eindeutigkeit der Zerlegung eines Systemes $S_{k}\left(a_{i}\right)$, dessen sämmtliche Elemente $a_{i}$ von Null verschieden sind, in Primsysteme, wobei unter einem Primsystem natürlich den Primzahlen entsprechend eine Reihe (I) zu verstehen ist, welche aus lauter Einheiten und einer einzigen Primzahl besteht.

Zwei Systeme $S_{k}\left(a_{i}\right)$ und $S_{k}\left(a_{i}^{\prime}\right)$ sollen als congruent nach dem Modul $S_{k}\left(m_{i}\right)$ bezeichnet werden, wenn die $k$ Congruenzen

$$
a_{i} \equiv a_{i}^{\prime}\left(\bmod m_{i}\right) \quad(i=1,2, \ldots, k)
$$

stattfinden und es soll dann, wie gewöhnlich,

$$
S_{k}\left(a_{i}\right) \equiv S_{k}\left(a_{i}^{\prime}\right) \quad\left(\bmod S_{k}\left(m_{i}\right)\right)
$$

geschrieben werden. Wie ohne Weiteres einleuchtet, erleiden bei dieser Erweiterung die fundamentalen Sätze über Congruenzen keine Änderung.

Nimmt man für die Vorschrift $\mathfrak{B}$ in den Forderungen 1. bis 4. der Nr. 1 des vorigen Absehnittes die Addition, so leistet die Gesammtheit von irgend welchen II $m_{i}$ modulo $S_{k}\left(m_{i}\right)$ incon$i=1$

gruenten Systemen diesen Bedingungen Genüge und bildet demnach hinsichtlich der Addition eine Abel'sche Gruppe.

Diese Gruppe ist wohl zu unterscheiden von einer zweiten bezüglich der Multiplication als Vorschrift $\mathfrak{B}$ gebildeten Abelschen Gruppe, welche sich aus der Gesammtheit von irgend welchen $i=k$

II $\varphi\left(m_{i}\right)$ nach dem Madul $S_{k_{k}}\left(m_{i}\right)$ incongruenten, mit $S_{k}\left(m_{i}\right)$ $i=1$
theilerfremden Systemen zusammensetzt. Hier ist wohl zu beachten, dass den Systemen nothwendig die einschränkende Eigenschaft, mit $S_{k}\left(m_{i}\right)$ theilerfremd zu sein, beigelegt werden musste, weil sonst der Forderung 2. 1. c. nicht Genüge geleistet worden wäre.

Obwohl nun natürlich für beide Gruppen die im vorigen Abschnitte für jede Vorschrift $\mathfrak{B}$ entwickelten Sätze Geltung besitzen, zeigen dieselben in beiden Fällen einen vollständig verschiedenen 
Charakter, weil eben als Vorschrift $\mathfrak{B}$ das einemal die Addition, das anderemal die Multiplication zu nehmen ist und darin liegt auch, wie man bei der speciellen Annahme $k=1$ und $m_{1}$ gleich einer Primzahl $p$ erkennt, der Grund, weshalb das vollständige Restsystem modulo $p$

$$
0,1,2, \ldots, \overline{p-1}
$$

sich wesentlich von dem Restsysteme

$$
1,2, \ldots, \overline{p-1}
$$

ohne die Null unterscheidet.

Es mag hier bemerkt sein, dass sich für die eben angeführten Gruppen ein weit über die im II. Abschn. entwickelten Sätze hinausgehendes Gebiet eröffinet, wenn man Ausdrücke betrachtet, die zu ihrer Bildung beide Operationen, sowohl die Multiplication, als auch die Addition benöthigen, eine Möglichkeit, welche den erwähnten Gruppen im Gegensatze zu andern, z B. den Substitutionsgruppen, einen eigenthümlichen Charakter verleiht.

So besteht z. B. der Satz, dass die Congruenz

$$
x^{n}+S_{k}\left(a_{i}^{(1)}\right) x^{n-1}+\cdots+S_{k}\left(a_{i}^{(n)}\right) \equiv 0 \quad\left(\bmod S_{k}\left(m_{i}\right)\right)
$$

$i=k$

II $n_{i}$ modulo $S_{k}\left(m_{i}\right)$ verschiedene Wurzeln besitzt, wenn die $i=1$

Congruenzen

$$
x^{n}+a_{i}^{(1)} x^{n-1}+\cdots+a_{i}^{(n)} \equiv 0 \quad\left(\bmod m_{i}\right) \quad(i=1,2, \ldots, k)
$$

der Reihe nach $n_{1}, n_{2}, \ldots, n_{k}$ verschiedene Wurzeln haben. Sind also die Module $m_{1}, \ldots, m_{k}$ Primzahlen, so kann die Congruenz (II) nicht mehr, als $n^{k}$ Wurzeln besitzen.

Selbstverständlich fallen aber diese und ähnliche Sätze über 7. zahlige Zahlsysteme bereits außerhalb des Rahmens dieser Arbeit, weshalb hier nicht näher auf sie eingegangen wird. Es sollen vielmehr in den folgenden Nummern die Entwickelungen des vorigen Abschnittes an der Hand des einfachsten Falles $k==1$ erläutert werden, wobei sich noch hinreichend Belege für die eben angeführte Bemerkung ergeben werden.

\section{Nr. 2. Das vollständige Restsystem.}

Die Abel'sche Gruppe, welche nach der vorigen Nummer irgend ein vollständiges Restsystem modulo $n$ in Bezug auf die Addition als Vorschrift $\mathfrak{B}$ bildet, ist regulär. Alle Elemente derselben lassen sich nämlich als ganzzahlige Vielfache eines einzigen von ibnen darstellen, da die Reihe

$$
0 . a, 1 . a, \ldots,(n-1) a
$$


falls $a$ und $n$ theilerfremd sind, ein vollständiges Restsystem modulo $n$ constituiert.

Die Null ist hier dasjenige Element, welches im vorigen Abschnitt als Einheit der Gruppe bezeichnet worden ist; denn das Hinzufügen der Null lässt jede Zahl ungeändert.

Die Wurzeln der Gleichung $X^{\delta}=1$ der Nr. 6 werden bei der betrachteten Gruppe nichts anderes als die Wurzeln der Congruenz $\delta x \equiv 0(\bmod x)$, d. h. die Zahlen

$$
0 \cdot \frac{n}{\tau}, 1 \cdot \frac{n}{\tau}, \cdots,(\tau-1) \frac{n}{\tau}
$$

sein, wenn mit $\approx$ der größte gemeinsame Theiler zwischen $n$ und $\delta$ bezeichnet wird und es ist nach dem Satze (4) der Nr. 5 klar, dass dieselben die einzige in einem vollständigen Restsystem modulo $n$ enthaltene Untergruppe vom Grade $\tau$ bilden, falls die Addition als Vorschrift $\mathfrak{B}$ genommen wird.

Ferner stimmen, falls der Modul $n$ durch $\delta$ theilbar vorausgesetzt wird, die $\varphi(\delta)$ Elemente, deren Grad $\delta$ ist, mit denjenigen Zahlen der Reihe $0,1, \ldots, \overline{n-1}$ überein, welche mit $n$ die Zahl $\frac{n}{\delta}$ zum gröbten gemeinsamen Divisor besitzen.

Endlich sei bemerkt, dass die in den Paragraphen 1 und 2 der Nr. 10 entwickelten Sätze über Combinationen auf die vorliegende Gruppe angewandt, die Theoreme des \$. 3 derselben Nr. bezüglich des Systemes $\overline{\mathbb{C}_{\delta}}$ ergeben.

Das Angeführte möge genügen, um die Art der Übertragung der allgemeinen Sätze des vorigen Abschnittes auf diesen speciellen Fall zu kennzeichnen.

Nr. 3. Über das vollständige mit $m$ theilerfremde Restsystem.

§. 1 .

Diejenige Abel'sche Gruppe $\mathrm{H}$, welche nach der $\mathrm{Nr} .1$ die Gesammtheit der $\varphi(m)$ zu $m$ theilerfremden Zahlen der Reihe

$$
0,1, \ldots, \overline{m-1}
$$

in Bezug auf die Multiplication bildet, ${ }^{1}$ ) ist bei beliebig zusammengesetztem Modul nicht regulär, und es handelt sich zunächst darum, ein Basissystem für diese Gruppe aufzustellen.

$\mathrm{Zu}$ diesem $\mathrm{Zwecke}$ zerlege man den vorerst als ungerade Zahl angenommenen Modul $m$ in seine Primfactoren, setze also

$$
m=\prod_{i=1}^{i=s} p_{i}^{\pi_{i}},
$$

1) Diese Abel'sche Gruppe findet sich bei Bachmann: Die Elemente der Zahlentheorie, 1892, pag. 58 angegeben. 
wo unter $p_{1}, \ldots, p_{s}$ ungerade Primzahlen zu verstehen sind. Sodann bestimme man eine Zahl $g_{i}(i=1,2, \ldots, s)$, welche den Congruenzen

$$
g_{i}=g_{i}^{\prime} \quad\left(\bmod p_{i}^{\pi_{i}}\right)
$$

und

(II) $\quad g_{i}=1 \quad\left(\bmod p_{j}^{\pi_{j}}\right)(j=1,2, \ldots, \overline{i-1}, \overline{i+1}, \ldots, s)$

Genüge leistet, in denen $g_{i}^{\prime}$ eine primitive Congruenzwurzel nach dem Modul $p_{i}^{\pi_{i}}$ bedeuten möge. Eine diese Bedingung erfüllende Zahl wird ersichtlich durch die Gleichung

$g_{i}=g_{i}^{\prime} \frac{m}{p_{i}^{\pi_{i}}} \mathrm{~A}_{i}+\frac{m}{p_{1}^{\pi_{1}}} \mathrm{~A}_{1}+\cdots+\frac{m}{p_{i=1}^{\pi_{i}-1}} \mathrm{~A}_{i-1}+\frac{m}{p_{i+1}^{\pi_{i}} \mathrm{~A}_{i+1}}+\cdots+\frac{m}{p_{s}^{\pi_{s}}} \mathrm{~A}_{s}$ angegeben, in welcher mit $A_{l}(l=1,2, \ldots, s)$ irgend eine Wurzel der Congruenz

$$
\frac{m}{p_{l}^{\pi_{l}}} x \equiv 1 \quad\left(\bmod p_{l}^{\pi_{l}}\right)
$$

bezeichnet ist.

Nach diesen Annanmen ist die Gesammtheit irgend welcher $\varphi(m)$ mit $m$ theilerfremden und modulo $m$ incongruenten Zahlen, oder wie kurz gesagt werden soll, ein vollständiges mit $m$ theilerfremdes Resteystem modulo $m$ in der Form

$$
\prod_{j=1}^{j=s} g_{j}^{\alpha_{j}} \quad\left(\alpha_{j}=0,1, \ldots, \overline{n_{j}-1} ; j=1,2, \ldots, s\right)
$$

darstellbar, wo unter $n_{j}$ die Gradzahl $\stackrel{p}{j=s}\left(p_{j}^{\pi_{j}}\right)$ des Basiselementes $g_{j}$ zu verstehen ist. Alle $\prod_{j=1} n_{j}$ oder $\varphi(m)$ Zahlen dieses Systemes sind nämlich erstens mit $m^{j=1}$ theilerfremd, zweitens untereinander in Bezug auf den Modul $m$ incongruent, weil aus der Annahme

$$
\prod_{j=1}^{j=s} g_{j}^{\alpha_{j}} \equiv \prod_{j=1}^{j=s} g_{j}^{\alpha^{\prime}}(\bmod m)
$$

dieselbe Congruenz auch modulo $p_{j}^{\pi} j$, also auf Grund der Relationen (II) die Beziehung $g_{j}^{\alpha} \equiv g_{j}^{\alpha}{ }^{{ }^{\prime}}\left(\bmod p_{j}^{\pi_{j}}\right), \mathrm{d}$, h. die Gleichung

für $j=1,2, \ldots, s$ folgt.

$$
\alpha_{j}=\alpha_{j}^{\prime}
$$


Natürlich bildet die Form (III) der Gruppe H eine Verallgemeinerung der Darstellung eines vollständigen Restsystemes ohne die Null in Bezug auf einen Primzahlmodul durch die Potenzen einer primitiven Congruenzwurzel.

Diese Betrachtungen erleiden eine bloß unwesentliche Modification im Falle eines geradzahligen Moduls.

Nimmt man erstens an, der Modul sei das Doppelte der ungeraden Zahl $m$, so hat man nur darauf zu achten, dass jede der primitiven Congruenzwurzeln $g_{1}, \ldots, g_{s}$ in dem Systeme (III) ungerade ist, eine Forderung, der, falls sie nicht schon von selbst erfüllt ist, unbeschadet der Bedingungen (I) und (II) stets dadurch Genüge geleistet werden kann, dass man die betreffenden primitiven Congruenzwurzeln um $m$ vermehrt. Der Complex (III) stellt dann nicht nur die obige Gruppe $H$ dar, sondern constituiert zugleich modulo $2 m$ ein vollständiges mit $2 m$ theilerfremdes Restsystem.

Besitzt zweitens der Modul die Form $4(2 \mu+1)$, so ändert sich an den vorstehenden Ausführungen ïberhaupt nichts, da die negative Einheit primitive Congruenzwarzel modulo 4 ist.

Hat aber endlich der Modul die Gestalt $2^{\lambda}(2 \mu+1)$, wo die Zahl $\lambda$. nicht kleiner als 3 angenommen wird, so muss der Umstand berủcksichtigt werden, dass es modulo $2^{2}$ keine primitiven Congruenzwurzeln gibt, sondern dass vielmehr zwei, zu den Exponenten 2, beziehungsweise $\frac{1}{2} \varrho\left(2^{\lambda}\right)$ gehörige Basiselemente, nämlich -1 und 5 nöthig sind, um alle ungeraden modulo $2^{2}$ incongruenten Zahlen in der bekannten Weise darzustellen. Dementsprechend hat man natürlich auch bei der Herleitung des dem Complexe (III) analogen Systemes für die Primzahlpotenz $2^{\lambda}$ zwei Basiselemente aufzustellen, welche jedoch im Übrigen analogen Bedingungen, wie sie für die anderen Basiselemente gelten, zu genügen haben.

Nachdem auf diese Weise für einen beliebigen Modul ein Basissystem gewonnen worden ist, können alle im vorigen $\mathrm{Ab}$ schnitte aufgestellten Sätze ohne Weiteres auf die vorliegende Abel'sche Gruppe $H$ übertragen werden. Es hat nur an die Stelle des Grades eines Elementes der Exponent, zu dem eine Zahl modulo $m$ gehört, zu treten und für jede Gleichung ist eine Congruenz mit dem Modul $m$ zu setzen. $Z u$ welchen Resultaten eine derartige Übertragung führt, mag die im Folgenden gegebene $\mathrm{Zu}$ sammenstellung von Sätzen darthun, wobei jedoch der einfacheren Ausdrucksweise halber der Modul $m$ als ungerade Zahl gleich $j=s$

$\prod_{j=1} p_{j}^{\pi_{j}}$ angenommen wird. 


$$
\text { §. } 2 .
$$

Während aus dem Satze (1) der Nr. 6 des vorigen Abschnittes folgt, dass jede lineare Congruenz, deren Coefficienten mit $m$ theilerfremd sind, eine und nur eine ebenso beschaffene Wurzel besitzt, entsprechen die beiden Sätze (2) und (6) a. a. O. dem verallgemeinerten Fermat'schen, bezw. Wils o n'schen Satze. Ferner erhält man, wenn man mit $\delta_{j}(j=1,2, \ldots, s)$ den größten gemeinsamen Theiler zwischen $\delta$ und $\varphi\left(p_{j}^{J_{j}}\right)$ bezeichnet, auf Grund der Sätze (3) and (5) 1. c. die beiden folgenden Theoreme:

Die Anzahl der Wurzeln der Congruenz

beträgt $\prod_{j=1}^{j=s} \delta_{j}$.

$$
x^{\delta} \equiv 1 \quad(\bmod m)
$$

Die gemeinsamen Wurzeln der beiden Congruenzen

$$
x^{\delta} \equiv 1 \text { und } x^{\delta \jmath^{\prime}} \equiv 1 \quad(\bmod m)
$$

(2) stimmen vollständig mit den Wurzeln der Congruenz

$$
x^{x} \equiv 1 \quad(\bmod m)
$$

überein, wenn unter $\tau$ der gröbte gemeinsame Divisorzwischen $\delta$ und $\delta^{\prime}$ verstanden wird.

Das Product aller Wurzeln der Congruenz (I) ist modulo $m$ mit der Einheit congruent; nur wenn für den Modul eine Primzahlpotenz und für $\delta$ eine gerade Zahl angenommen wird, ergibt sich dasselbe congruent mit -1 .

Die Summe derselben Wurzeln findet man mit dem Producte

$$
\prod_{=1}^{j=s} \frac{g_{j}^{\varphi\left(p_{j}^{\pi_{j}}\right)}-1}{\frac{\varphi^{\left(p_{j}^{\pi_{j}}\right)}}{g_{j}}-1}
$$

modulo $m$ congruent, d. h. durch $m$ theilbar, wenn $\delta$ mit keiner der Zahlen $p_{j}-1(j=1,2, \ldots, s)$ theilerfremd ist. Speciell für einen geraden Exponenten o trifft dies immer zu und man kann in diesem Falle sogar den Wert der Summe der Wurzeln der Congruenz (I), die kleiner als $m$ sind, angeben. Ordnet man nämlich einer jeden solchen Wurzel $p$ eine andere mit ihr incongruente $m-\rho \mathrm{zu}$, so erhält man für die gesuchte Summe den Ausdruck

$$
\frac{1}{2} m \prod_{j=1}^{j=s} \delta_{j}
$$


welcher für den besonderen Fall $\delta=\varphi(m)$ in die bekannte Summe $\frac{m \varphi(n)}{2}$ aller zu $m$ theilerfremden positiven Zahlen, die kleiner als $m$ sind, übergeht.

Die beiden Sätze (1) und (2) reichen nach den Ausführungen der Nr. 7 im II. Abschn. aus, um mit Hilfe der Methode des Ausscheidens und Hinzufügens die Anzahl $\bar{\varphi}\left(\delta ; p_{1}^{\pi_{1}-1}\left(p_{1}-1\right)\right.$, $\left.p_{2}^{\pi_{2}-1}\left(p_{2}-1\right), \ldots, p_{s}^{\tau_{s}-1}\left(p_{s}-1\right)\right)$ der Zahlen, die zum Exponenten $\delta$ modulo $m$ gehören, zu bestimmen.

Auf Grund des Satzes (1) 1. c. erhält man das folgende Theorem:

Ist die Zahl $\delta$ in ihre Primfactoren zerlegt, gleich dem Producte $\prod_{i=1}^{i=r} q_{i}^{k_{i}}$, so wird die Anzahl der modulo m incongruenten Zahlen, die zum Exponenten $\delta$ gehören, durch den Ausdruck

$$
\prod_{j=1}^{j=s} \delta_{j} \cdot \prod_{i=1}^{i=r}\left(1-\frac{1}{q_{i}^{l} q_{i}}\right)
$$

bestimmt, wo unter $l_{q_{i}}(i=1,2, \ldots, r)$ die Anzahl derjenigen der Zahlen $\varphi\left(p_{1}^{\pi_{1}}\right), \ldots, \varphi\left(p_{s}^{\pi_{s}}\right)$ zu verstehen ist, welcheden Primfactor $q_{i}$ in nicht geringerer Potenz enthalten, wie der Exponent ò.

Speciell folgt hieraus der bekannte Satz: Es gibt keine Zahl, die modulo $m$ zum Exponenten $\rho(m)$ gehört, d. h. die Gruppe $H$ ist nicht regulär, wenn der ungerade Modul $m$ verschiedene Primfactoren enthält.

Das Product aller Zahlen, die zum Eponenten ò gehören, ist nach dem Satze (6) 1. c. congruent mit der Einheit modulo m, ausgenommen in dem Falle $\delta=2$ and $m=p_{1}^{\pi_{1}}$, in welchem nur die negative Einheit zum Exponenten 2 gehört.

Mit Hilfe des Ausdruckes (I) a. a. O. lässt sich die Summe aller Zahlen, die zum Exponenten ô gehören, unter der Voraussetzung modulo $m$ bestimmen, dass der Quotient $\frac{\delta}{i=r}$ mit keiner

$$
\prod_{i=1} q_{i}
$$

der Zahlen $\overline{p_{1}-1}, \ldots, \overline{p_{s}-1}$ theilerfremd ist. Dann wird dieselbe auf Grund des Ausdruckes (II) dieses Paragraphen durch $m$ theilbar. Speciell für die Summe aller positiven Zahlen, die zum Exponenten $\delta$ gehören und kleiner als $m$ sind, erhält man, vorausgesetzt, dass die Zahl $\delta$ den Divisor 4 besitzt, entsprechend dem Producte (III) 
den Wert $\frac{1}{2} m \bar{\varphi}\left(\bar{\delta} ; p_{1}^{\pi_{1}-1}\left(p_{1}-1\right), \ldots, p_{s}^{* \tau_{s}-1}\left(p_{s}-1\right)\right)$, ein Resultat, welches sich in einfacher Weise auch durch die Bemerkung bestätigen lässt, dass bei der gemachten Annahme die beiden Zahlen $p$ und $-p$ gleichzeitig zum Exponenten $\delta$ gehören oder nicht.

\section{§. 3.}

Bekanntlich heißt die mit $m$ theilerfremde Zahl $a$ ein $\delta^{\text {ter }}$ Potenzrest oder kurz ein ${ }^{\text {ter }}$ Rest modulo $m$, wenn die Congruenz

$$
x^{\delta} \equiv a \quad(\bmod m)
$$

wenigstens eine Wurzel besitzt.

Die Gesammtheit der $\delta^{\text {ten }}$ Potenzreste modulo $m$ stimmt also mit der Untergruppe der $\delta^{\text {ten }}$ Potenzen der Gruppe $\mathrm{H}$ überein und man erhält entsprechend den Theoremen (7), (8) and (9) der Nr. 6 die drei folgenden Sätze:

Die Anzahl der $\delta^{\text {ten }}$ Potenzreste modulo $m$ be(1) $\operatorname{trägt} \frac{\varphi(m)}{j=s}$.

$$
\prod_{j=1} \hat{o}_{j}
$$

Die $\delta^{\text {ten }}$ und $\delta^{\prime \text { ten }}$ Reste sind identisch, wenn (2) die beiden Zahlen $\delta$ und $\delta^{\prime}$ mit $\varphi(m)$ denselben größten gemeinsamen Theiler besitzen. überein,

So stimmen z. B. die $\delta^{\text {ten }}$ und $(-\delta)^{\text {ten }}$ Reste miteinander

Diejenigen Zahlen, welche gleichzeitig $\delta^{\text {te }}$ und $\delta^{\prime \text { te }}$ Reste sind, bilden die Gesammtheit der $\left(\frac{\delta \delta^{\prime}}{\tau}\right)^{\text {ten }}$

Reste, wenn mit $\tau$ der größte gemeinsame Divisor $z$ wischen $\delta$ und $\delta^{\prime}$ bezeichnet wird.

Das Product der $\delta^{\text {ten }}$ Potenzreste ist modulo $m$ congruent mit +1 , vorausgesetzt, dass nicht einer der beiden folgenden Fälle vorliegt:

1. Der Modul $m$ ist eine Primzahlpotenz $p_{1}^{\pi_{1}}$ und die Differenz $\overline{p_{1}-1}$ enthält den Factor 2 in höherer Potenz, wie der Exponent $\delta$.

2. Der Modul $m=\prod p_{j}^{\pi_{j}}$ setzt sich aus 2 oder mebreren Primfactoren $p_{1}, \ldots, p_{s} \begin{gathered}j=1 \\ \text { von den Formen }\end{gathered}$

$$
1+2^{\alpha_{1}}\left(1+2 \mu_{1}\right) ; \text { bezw. ... } 1+2^{\alpha_{s}}\left(1+2 \mu_{s}\right)
$$

zusammen und es bestehen die Relationen

$\alpha_{1}>\alpha_{2} \geq \alpha_{3} \geq \cdots \geq \alpha_{s} ; \delta \equiv 0\left(\bmod 2^{\alpha_{2}}\right) ; \delta$ nicht $\equiv 0\left(\bmod 2^{\alpha_{1}}\right)$.

Monatsh. f. Mathematik u. Physik, VIJ. Jahrg. 
In diesen beiden Föllen wird das Product congruent mit -1 modulo $p_{1}^{\pi_{1}}$ und congruent mit +1 modulo $\frac{m}{p_{1}^{\pi_{1}}}$.

Die Summe der $\delta^{\text {ten }}$ Potenzreste findet man modulo $m$ mit dem Producte

$$
\prod_{j=1}^{j=s} \frac{g_{j}^{\varphi\left(p_{j}^{\tau j}\right)}-1}{g_{j}^{\delta_{j}}-1}
$$

congruent, d. h. durch $m$ theilbar, sobald $\delta$ weder ein Vielfaches von $\overline{p_{1}-1}$, noch von $\overline{p_{2}-1}$, noch von $\ldots \overline{p_{s}-1}$ ist. Diese Bedingung ist sicher für jede ungerade Zahl $\delta$ erfüllt, und da in diesem Falle die negative Einheit unter den $\delta^{\text {ten }}$ Resten vorkommt, bilden die positiven $\delta^{\text {ten }}$ Reste, welche kleiner als $m$ sind, ihrer Größe nach geordnet eine Reihe der Form

$$
r_{1}, \ldots, r_{k}, \overline{m-r_{k}}, \ldots, \overline{m-r_{1}} \quad\left(\begin{array}{r}
k=\frac{\varphi(m)}{j=s} \\
2 \prod_{j=1}^{j=s} \delta_{j}
\end{array}\right)
$$

und haben mithin den Wert $\frac{m \varphi(m)}{j=s}$ zur Summe.

$$
2 \prod_{j=1} \delta_{j}
$$

Nimmt man in dem Ausdrucke (IV) 1. c. die Zahlen $\bar{\delta}_{1}, \ldots, \overline{\delta_{r}}$ ungerade an, so erkennt man auf Grund der eben durchgeführten Betrachtungen, dass die Summe der Zahlen, die weder $\bar{\delta}_{1}^{\text {te }}$, noch $\ldots \bar{\delta}_{r}^{\text {te }}$ Reste sind, congruent mit Null, ihr Product hingegen bei zusammengesetztem Modul congruent mit der positiven Einheit modulo $m$ ist, vorausgesetzt natürlich, dass überhaupt solche Zahlen existieren.

Soll die Analogie, welche zwischen der Gesammtheit $\Gamma_{\delta}$ der $\delta^{\text {ten }}$ Potenzreste einerseits, und der Gesammtheit der Wurzeln einer Congruenz der Form $x^{\delta} \equiv 1(\bmod m)$ andererseits besteht, eine vollständige sein, so müssen sich auch zu den Zahlen, die zum Exponenten ò gehören, analoge Individuen innerhalb der Gruppe $\Gamma_{\delta}$ auffinden lassen.

Um dieser Frage näher treten zu können, sollen im Folgenden nur solche Gruppen $\Gamma_{\delta}$, bei denen der Index $\delta$ ein Theiler von $\varphi(m)$ ist, betrachtet werden. Dies kann ohne Einschränkung der Allgemeinheit auf Grund des Satzes (2) geschehen; existieren ja doch nach diesem Satze nur soviele verschiedene Arten von $\delta^{\text {ten }}$ Resten, als es Theiler $\delta$ von $\varphi(m)$ gibt. 
Es leuchtet ohne Weiteres ein, dass jeder $(k \hat{\delta})^{\text {te }}$ Rest von selbst gleichzeitig $\delta^{\text {ter }}$ Rest ist. Es können mithin $\delta^{\text {te }}$ Reste zugleich höhere Reste sein, so dass das Problem entsteht:

Es soll die Gesammtheit $P_{\delta}$ derjenigen Elemente angegeben werden, welche zwar $\delta^{\text {te }}$, aber keine höheren Potenzreste sind.

Hierbei ist wohl zu beachten, dass unter "höheren" Potenzresten solche $(k \delta)^{\text {te }}$ Reste $z u$ verstehen sind, bei denen die Zahl $k \delta$ Divisor von $\varphi(m)$ ist. Die Individuen des gesuchten Complexes $P_{\delta}$ lassen sich nun dadurch charakterisieren, dass sie wobl der Gruppe $\Gamma_{\delta}$, aber keiner der Gruppen $\Gamma_{\delta q_{i}}(i=1,2, \ldots, r)$ angehören, wo mit $q_{1}, \ldots, q_{s}$ die verschiedenen Primfactoren des Quotienten $\frac{\stackrel{(m)}{\delta}}{\delta}$ bezeichnet worden sind. Jede solche Zahl $a$ ist nämlich stets und nur dann zugleich $\delta^{\prime \text { ter }}$ Rest, wenn $\delta^{\prime}$ Theiler von $\delta$ ist. Denn sie gehört unter dieser Annahme simultan den beiden Complexen $\Gamma_{\delta}$ und $\Gamma_{\delta^{\prime}}$, also nach dem Satze (3) auch der Gruppe $\Gamma_{\frac{\delta \delta^{\prime}}{\tau}}$ an. Wäre nun $\tau$ nicht gleich $\delta^{\prime}$, so wïrde das Individuum $a$ gegen die Annahme mindestens einer der Gruppen $\Gamma_{\delta q_{i}}(i=1, \ldots, r)$ angehören müssen.

Auf Grund dieser Bemerkung findet man mit Hilfe der Methode des Ausscheidens und Hinzutügens gemäß dem Satze (3) die Beziehung

$$
P_{\delta}=\Gamma_{\delta} \cdot \dot{\Sigma} \Gamma_{\delta q} \dot{i} \dot{\Sigma}_{q q^{\prime}} \Gamma_{\delta q q^{\prime}} \doteq \cdots
$$

Hieraus ergibt sich nach dem Satze (1) für die Anzahl $\psi\left(\delta ; p_{1}^{\pi_{1}-1}\left(p_{1}-1\right), \ldots, p_{s}^{\pi_{s}-1}\left(p_{s}-1\right)\right)$ der Individuen von $P_{\delta}$ der Ausdruck

$$
\varphi(m)\left\{\frac{1}{j=s}-\sum \frac{1}{j=s}+\sum_{j=1} \frac{1}{\prod_{j=1}(\delta q)_{j}}-\cdots\right\}
$$

aus welchem man das folgende Theorem erschließt:

Die Anzahl derZahlen, die modulo $m \delta^{\text {te }}$ und keine höheren Potenzreste sind, wird durch den A usdruck

$$
\prod_{j=1}^{\varphi(m)} \prod_{j}^{i=s} \delta_{i=1}^{i=r}\left(1-\frac{1}{q_{i}^{h} q_{i}}\right)
$$

bestimmt, wo $h_{q_{i}}$ die Anzahl der Zahlen $p_{j}^{\pi_{j}-1}\left(p_{j}-1\right)$ $(j=1, \ldots, s)$ angibt, welche den Primther er $q_{i}(i=1, \ldots, r)$ 
von $\frac{\vartheta(m)}{\delta}$ in höherer Potenz enthalten, wie der Exponent $\delta$.

Bezeichnet man also mit $w$ das kleinste gemeinsame Vielfache der $s$ Zahlen $\varphi\left(p_{1}^{\pi_{1}}\right), \ldots, \varphi\left(p_{s}^{\pi_{s}}\right)$ und mit $t$ denjenigen größten Divisor von $\delta$, der zu $\frac{\rho(m)}{\delta}$ relativ prim ist, so gibt es dann und nur dann derartige Zahlen, wenn das Product $\frac{\grave{\delta}}{t} q_{1} \cdots q_{r}$ ein Theiler von $w$ ist. Dieser Fall tritt immer ein, wenn die beiden Zahlen $\delta$ und $\frac{\varphi(m)}{\delta}$ theilerfremd sind.

Weil jede Zahl sich in einem und nur in einem Complexe $P_{\delta}$ vorfindet, besteht analog dem Satze (4) in der Nr. 7 die Gleichung

$$
\sum_{\delta} \psi\left(\grave{o} ; p_{1}^{\pi_{1}-1}\left(p_{1}-1\right), \ldots, p_{s}^{\pi_{s}-1}\left(p_{s}-1\right)\right)=\emptyset(m),
$$

in der sich o über alle Theiler von $\varphi(m)$ zu erstrecken hat.

Aus dem Ausdrucke (I) lassen sich ohne Weiteres auch die Summe und das Product der ôten und nicht höheren Potenzreste modulo $m$ bestimmen. Man findet die erstere durch $m$ theilbar, falls keine der $s$ Zahlen $\overline{p_{1}-1}, \ldots, \overline{p_{s}-1}$ ein Divisor des Productes $\delta q_{1} \ldots q_{r}$ ist, das letztere hingegen congruent mit +1 , wenn sich nicht der Quotient $\frac{\varphi(m)}{\delta}$ auf eine Potenz von 2 reduciert.

Schließlich sei noch bemerkt, dass im Falle $m=p^{\pi}$ die Zahlen $a$, welche $\delta^{\text {te }}$ und nicht höhere Reste sind, vollständig mit den Zahlen, die zum Exponenten $\frac{\varphi(m)}{\delta}$ gehören, übereinstimmen; denn dann besteht erstens die Gleichung

$$
\psi\left(\delta ; p^{\pi-1}(p-1)\right)=\varphi\left(\frac{\varphi\left(p^{\pi}\right)}{\delta}\right),
$$

zweitens die Beziehung

$$
a^{\frac{\varphi\left(p^{\pi}\right)}{\delta}} \equiv 1 \text {, aber nicht } a^{\frac{\varphi\left(p^{\pi}\right)}{\delta q_{i}}} \equiv 1\left(\bmod p^{\pi}\right)(i=1,2, \ldots, r),
$$

da sonst der Index von $a$ durch $\delta q_{i}$ theilbar, also die Zahl $a$ gegen die Annahme ein $\left(\delta q_{i}\right)^{\text {ter }}$ Potenzrest wäre.

Nach diesen Bemerkungen über die Zahlen, , welche $\hat{o}^{\text {te }}$ und nicht höhere Potenzreste sind, möge mit der Übertragung der Sätze der $\mathrm{Nr}$. 6 des vorigen Abschnittes fortgefahren werden. 
Dem Theoreme (10) 1. c. zufolge sind die $\delta^{\text {ten }}$ Potenzreste, falls unter $v$ das kleinste gemeinschaftliche Vielfache von $\frac{n_{1}}{\delta_{1}}, \cdots, \frac{n_{s}}{\delta_{s}}$ verstanden wird, dann und nur dann identisch mit den Wurzeln der Congruenz $x^{v} \equiv 1(\bmod m)$, wenn jeder Primtheiler von $\delta$, der wenigstens in zwei der Zahlen $p\left(p_{1}^{\pi_{1}}\right), \ldots, p\left(p_{s}^{\pi_{s}}\right)$ in verschiedener Potenz aufgeht, die Zahl $\delta$ in einer Potenz theilt, welche nicht kleiner ist, als die höchste Potenz, mit der er in $w$ aufgeht. Unter dieser Annahme hat die Congruenz

$$
x^{\delta} \equiv a \quad(\bmod m)
$$

entweder genau $\prod_{j=1}^{j=s} \delta_{j}$ Wurzeln oder überhaupt keine, je nachdem die Beziehung

besteht, oder nicht.

$$
a^{v} \equiv 1 \quad(\bmod m)
$$

Der bezeichnete Fall tritt immer ein, sobald der Modul $m$ eine Primzahlpotenz $p^{z}$ ist. Die Gruppe $\mathrm{H}$ and damit auch die Untergruppe $\Gamma_{\delta}$ werden dann regulär, und zwar bilden die $\delta^{\text {ten }}$ Potenzreste die einzige Untergruppe von $\mathrm{H}$ bezüglich der Multiplication vom Grade $\frac{p^{\pi t}(p-1)}{\tau}$, wo $\tau$ den größten gemeinsamen Theiler zwischen $p\left(p^{\pi t}\right)$ and $\delta$ bedeutet.

Setzt man der Einfachheit halber wieder $\tau=\delta$ voraus, so nimmt das System $\left(\mathfrak{U}_{\delta}\right)$ l. c. die Gestalt

$$
\begin{aligned}
& 1, g^{\delta}, \ldots, g^{\left(\frac{\varphi\left(p^{\delta}\right)}{\delta}-1\right) \delta} \\
& \cdot \cdot \cdot \cdot \cdot \cdot \cdot \cdot \cdot \cdot \cdot \\
& g^{\delta-1}, g^{\overline{\delta-1}+\delta}, \ldots, g^{\delta-1}+\left(\frac{\varphi\left(p^{\tau}\right)}{\delta}-1\right) \delta
\end{aligned}
$$

an, falls unter $g$ eine primitive Congruenzwurzel modulo $p^{3 x}$ verstanden wird. Schreibt man in diesem schon ron Gauss in der Theorie der Kreistheilung im Faile $\pi=1$ verwendeten Schema für jede Zahl ihren kleinsten positiven Rest modulo $p^{\pi}$ und ordnet man in den einzelnen Klassen die Reste ihrer Größe nach, so erhält man eine völlig bestimmte Eintheilung

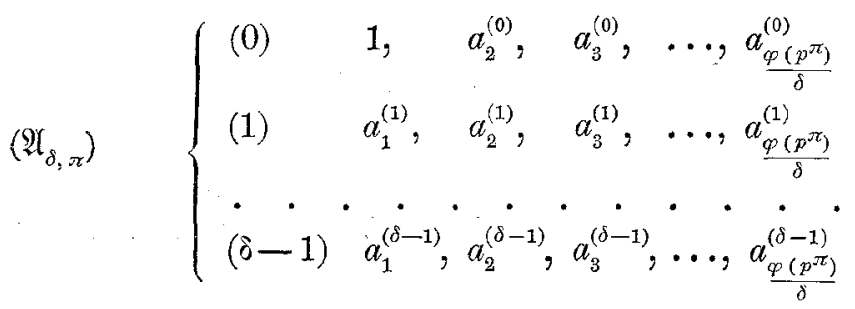


der Zahlen

$1,2, \ldots, \overline{p-1}, \overline{p+1}, \overline{p+2}, \ldots, \overline{2 p-1}, \overline{2 p+1}, \ldots, \overline{p^{\tau}-1}$

in $\delta$ Klassen, deren Anordnung freilich von der Wahl der primitiven Congruenzwurzel abhängt (vergl. Nr. 6).

Während sich die Betrachtungen in der eben erwähnten Nr. auf das Verhältnis zweier Systeme $\left(\mathfrak{A}_{\delta, \pi}\right)$ und $\left(\mathfrak{A}_{\delta^{\prime}, \pi}\right)$ bei gleichbleibendem Modul beziehen, möge hier die Abhängigkeit des Schemas $\left(\mathfrak{U}_{\delta, \pi}\right)$ von dem Potenzexponenten $\pi$ des Moduls näher untersucht werden und zwar unter der Voraussetzung, dass die Zahl $\delta$ den Primfactor $p$ nicht enthält.

Zunächst ist klar, dass jede Zahi der Klasse $(i)$ im Schema $\left(\mathfrak{A}_{\delta, \pi}\right)$ einer Zahl derselben Klasse des für den Modul $p$ und dieselbe primitive Congruenzw urzel gebildeten Schemas $\left(\mathfrak{A}_{\delta, 1}\right)$ modulo $p$ congruent sein muss. Es folgt nämlich unmittelbar aus der Congruenz

$$
\left(a_{k}^{(i)}\right)^{\frac{p^{r t-1}(p-1)}{\delta}} \equiv g^{i \frac{p^{\pi-1}(p-1)}{\delta}}\left(\bmod p^{\pi}\right)
$$

auf Grund der für jede Zahl $z$ giltigen Beziehung $z^{p} \equiv z(\bmod p)$ auch die Congruenz

$$
\left(a_{k}^{(i)}\right)^{\frac{p-1}{\delta}} \equiv g^{i \frac{p-1}{\delta}}(\bmod p)
$$

welche den Nachweis für die gemachte Behauptung liefert, weil bekanntlich jede primitive Congruenzwurzel modulo $p^{\pi}$ auch primitive Congruenzwurzel modulo $p$ ist.

Umgekehrt gehört aber auch jede Zahl $\bar{a}_{k}^{(i)}$ der $i^{\text {ten }}$ Klasse des Schemas $\left(\mathfrak{H}_{\delta, 1}\right)$ derselben Klasse im Schema $\left(\mathfrak{T}_{\delta, \pi}\right)$ an. Denn ans der Beziehung

d. h.

$$
\left(\bar{a}_{k}^{(i)}\right)^{\frac{p-1}{\delta}} \equiv g^{i \frac{p-1}{\delta}}(\bmod p)
$$

$$
\left(a_{k}^{(i)}\right)^{\frac{p-1}{\delta}}=g^{i \frac{p-1}{\delta}}+M p
$$

folgt zunächst die Relation

$$
\left(\bar{a}_{k}^{(i)}\right)^{\frac{p}{\overline{p-1}}}=g^{i p \frac{p-1}{\delta}}+\mathrm{M}^{\prime} p^{2}
$$

in der $M^{\prime}$ eine ganze Zahl bedeutet, wie man sofort erkennt, wenn man die Gleichung (I) auf die $p^{\text {te }}$ Potenz erhebt und die rechte Seite nach dem binomischen Satz entwickelt. Verfährt man mit der 
Gleichung (II) ebenso, wie mit der Gleichung (I) u. s. f., so gelangt man schließlich zur Congruenz

$$
\left(a_{k}^{(i)}\right)^{p^{\pi-1} \frac{p-1}{\delta}} \equiv g^{i p^{\pi-1} \frac{p-1}{\delta}}\left(\bmod p^{\pi}\right),
$$

welche das Kriterium dafür abgibt, dass die Zahl $\vec{a}_{k}^{(i)}$ der $i^{\text {ten }}$ Klasse im Schema $\left(\mathfrak{A}_{\delta, \pi}\right)$ angehört. Es lassen sich somit die Zahlen der $i^{\text {ten }}$ Zeile im Schema $\left(\mathfrak{A}_{\delta, \pi}\right)$ in die Anordnung

$a_{1}^{(i)}+k p, a_{2}^{(i)}+k p, \ldots, a_{\frac{p-1}{\delta}}^{(i)}+k p \quad\left(k=0,1, \ldots,\left(p^{\pi-1}-1\right)\right)$ bringen, wobei die Reihe $a_{1}^{(i)}, \ldots, a_{\frac{p-1}{\delta}}^{(i)}$ genau die $i^{\text {te }}$ Zeile im Schema
$\left(\mathfrak{H}_{\delta, 1}\right)$ ausmacht.

Man kann sich daher, wie dies auch im Folgenden geschehen soll, auf Primzahlmoduln beschränken.

Das Schema $\left(\mathfrak{U}_{\delta, 1}\right)$ bildet eine Verallgemeinerung der für $\delta=2$ allgemein gebrauchten Klassificierung des vollständigen Restsystemes modulo $p$ (excl. 0 ) in quadratische Reste und quadratische Nichtreste. Wie in diesem speciellen Falle gemäß dem Euler'schen Kriterium das Bestehen der Congruenz

$$
a^{\frac{p-1}{2}} \equiv 1 \text { oder } a^{\frac{p-1}{2}} \equiv g^{\frac{p-1}{2}}(\bmod p)
$$

entscheidet, ob die Zahl a quadratischer Rest oder Nichtrest ist, ebenso gehört nach den Ausführungen der Nr. 6 die Zahl a dann und nur dann im Schema $\left(\mathfrak{H}_{\delta, 1}\right)$ der Klasse $(j)$ an, wenn die Congruenz

$$
a^{\frac{p-1}{\delta}} \equiv g^{j \frac{p-1}{\delta}}(\bmod p)
$$

erfüllt ist. Man kann auch hier ein dem in der Theorie der quadratischen Reste auftretenden Legendre'schen Symbol analoges Zeichen

$$
\left(\frac{a}{p}\right)_{\delta}
$$

einführen; und zwar wird unter diesem Zeichen entsprechend der Congruenz (III) die Zahl $r_{j}$ zu verstehen sein, wenn die dem Betrage nach den Wert $\frac{p}{2}$ nicht übertreffenden positiven und negativen Reste aller $\left(\frac{p-1}{\delta}\right)^{\text {ten }}$ Potenzreste

$$
1, g^{\frac{p-1}{\delta}}, \ldots, g^{(\delta-1) \frac{p-1}{\delta}}
$$

der Reihe nach mit $r_{0}, r_{1}, \ldots, r_{\delta-1}$ bezeichnet werden. 
Ohne auf diesen Gegenstand weiter einzugehen, sei hier nur hervorgehoben, dass für dieses das Legendre'sche Symbol verallgemeinernde Zeichen die beiden folgenden Sätze erhalten bleiben:

1. Aus der Congruenz

folgt die Gleichung

$$
a \equiv a^{\prime}(\bmod p)
$$

$$
\left(\frac{a}{p}\right)_{\delta}=\left(\frac{a^{\prime}}{p}\right)_{\delta}
$$

2. Es besteht die Congruenz

$$
\left(\frac{a a^{\prime}}{p}\right)_{\delta} \equiv\left(\frac{a}{p}\right)_{\delta}\left(\frac{a^{\prime}}{p}\right)_{\delta}(\bmod p)
$$

Aus der letzten Bemerkung ergibt sich der Satz:

Der Klassenzeiger einer zusammengesetzten Zahl für das Schema $\left(\mathfrak{H}_{\delta, 1}\right)$ ist modulo $\delta$ gleich der Summe der Klassenzeiger aller ihrer gleichen und ungleichen Primfactoren.

\section{§. 4 .}

$\mathrm{Zu}$ den in der Nr. 9 angeführten Sützen kann man bei der hier behandelten Gruppe das folgende Theorem hinzufügen:

Besitzen die modulo $m$ incongruenten und mit $m$ theilerfremden Zahlen

$$
a_{1}, a_{2}, \ldots, a_{k}
$$

einen von der Einheit verschiedenen Multiplicator $\mathfrak{m}$, (1) welcher bezüglich des Primfactors $p_{j}$ von $m \mathrm{zum}$ Exponenten $\Theta_{j}(j=1,2, \ldots, s)$ gehört, so ist jede aus diesen Elementen gebildete homogene symmetrische Function, deren Grad durch keineder Zahlen $\theta_{1}, \ldots, \theta_{s}$ theilbar ist, ein Multiplum ron $m .{ }^{1}$ )

Es möge die zu untersuchende homogene, symmetrische Function der Elemente der Reihe (I) mit $f\left(a_{1}, \ldots, a_{k}\right)$, ihr Grad aber mit $\lambda$ bezeichnet werden. Dann besteht nach der Definition eines Multiplicators des Systemes (I) (vergl. Nr. 9) die Congruenz $f\left(\mathfrak{n t} a_{1}, \ldots, \mathfrak{n t} a_{k}\right) \equiv f\left(a_{1}, \ldots, a_{k}\right)$ oder

2.

$$
\mathfrak{m}^{\lambda} f\left(a_{1}, \ldots, a_{k}\right) \equiv f\left(a_{1}, \ldots, a_{k}\right) \quad(\bmod m),
$$

1) Ein analoger Satz besteht natiírich für die aus der Einheit und den $n_{\text {ten }}$ Einheitswurzeln zusammengesetzten symmetrischen Functionen. 
aus welcher, falls unter $t$ der gröbte gemeinsame Theiler zwischen der Zahl $f\left(a_{1}, \ldots, a_{k}\right)$ und dem Modul $m$ verstanden wird, die Beziehung

$$
\mathfrak{H t}^{2} \equiv 1 \quad\left(\bmod \frac{m}{t}\right)
$$

folgt. Enthielte num $\frac{m}{t}$ einen Primfactor $p_{j}$, so müsste wegen der Congruenz $\mathfrak{m}^{\lambda} \equiv 1\left(\bmod p_{j}\right)$ die Zahl $\lambda$ ein Vielfaches des Exponenten $\Theta_{j}$ sein, zu welchem der Multiplicator $\mathfrak{n}$ modulo $p_{j}$ gehört, was gegen die Annahmen des Satzes (1) verstößt. Der Quotient $\frac{m}{t}$ muss also gleich der Einheit und damit die Function $f\left(a_{1}, \ldots, a_{k}\right)$ durch $m$ theilbar sein.

So wird z. B., falls die Zahl $\delta$ als Theiler von $\overline{p-1}$ vorausgesetzt wird, jede aus den Zahlen irgend welcher Klassen des Schemas $\left(\mathfrak{H}_{\delta, \pi}\right)$ im vorigen Paragraphen gebildete, homogene, symmetrische Function, deren Grad nicht durch $\frac{p-1}{\delta}$ theilbar ist, ein Multiplum von $p^{\pi}$ sein müssen; denn jede Klasse von $\left(\mathfrak{A}_{\delta, \pi}\right)$ besitzt den Multiplicator $g^{\delta}$, der modulo $p$ zum Exponenten $\frac{p-1}{\delta}$ gehört. Speciell wird also jede aus den Elementen

$$
k p+r \quad\left(k=0,1, \ldots,\left(p^{\pi-1}-1\right) ; r=1,2, \ldots,(p-1)\right)
$$

gebildete homogene symmetrische Function, deren Grad kein Multiplum von $\overline{p-1}$ ist, durch $p^{\pi}$ theilbar sein.

Ferner muss eine jede aus den Elementen eines vollständigen mit $m$ theilerfremden Restsystemes gebildete homogene symmetrische Function ungeraden Grades durch die ungerade Zahl $m$ theilbar sein; denn das angeführte Zahlsystem hat -1 zum Multiplicator.

Den Bedingungen des Satzes (1) kann übrigens, falls die Differenz $\mathfrak{H}-1$ relativ prim zu $m$ ist, dadurch Genüge geleistet werden, dass der Grad $\lambda$ der Function $f\left(a_{1}, \ldots, a_{k}\right)$ theilerfremd mit dem größten gemeinsamen Divisor $\Theta$ zwischen der Anzahl $k$ der Elemente und dem kleinsten gemeinschaftlichen Vielfachen der Zahlen $\overline{p_{1}-1}, \ldots, \overline{p_{s}-1}$ angenommen wird; der Exponent $\theta_{\mathcal{J}}$ theilt nämlich nicht nur die Differenz $\overline{p_{j}-1}$, sondern anch die Anzahl $k$, weil nach dem Satze (1) l. c. die Zahl $k$ sogar ein Multiplum desjenigen Exponenten ist, zu dem der Multiplicator m modulo $m$ gehört; außerdem muss natürlich jede Zahl, welche simultan in $\overline{p_{j}-1}$ und $k$ autgeht, auch $\Theta$ theilen.

Zum Schlusse dieser Nr. mag noch bemerkt sein, dass die in der Nr. 10 entwickelten Betrachtungen über Combinationen in 
ihrer Anwendung auf die vorliegende Gruppe $H$ auch als Untersuchungen über die Lösungssysteme $x_{1}, \ldots, x_{\delta}$ der Congruenz

$$
x_{1} x_{2} \ldots x_{\delta} \equiv j(\bmod m)
$$

aufgefasst werden können, wobei keine zwei Unbekannten $x_{i}$ und $x_{i^{\prime}}$ $\left(i, i=1,2, \ldots, \delta ; i\right.$ nicht $\left.=i^{\prime}\right)$ einander gleich angenommen werden.

Nr. 4. Die $\delta^{\text {ten }}$ Potenzreste werden als Gruppe für sich betrachtet.

$\S .1$.

Während im §. 3 der vorigen $\mathrm{Nr}$. die Gesammtheit $\Gamma_{\delta}$ der $\delta^{\text {ten }}$ Potenzreste modulo $m$ wesentlich in ihrem Verhältnis zu dem vollständigen mit $m$ theilerfremden Restsystem betrachtet worden ist, soll dieselbe in der vorliegenden Nr. für sich einer Untersuchung unterzogen werden; es ist nämlich klar, dass sich alle im vorigen Abschnitte entwickelten Sätze auch auf dieses System übertragen werden, weil es als Untergruppe einer Abel'schen Gruppe selbst eine solche bezüglich der Multiplication darstellt.

Unter Berücksichtigung des Umstandes, dass bei Beibehaltung der Bezeichnungen der vorigen Nr. die Elemente $g_{1}^{\delta_{1}}, \ldots, g_{s}^{\delta_{s}}$ mit den Gradzahlen $\frac{\varphi\left(p_{1}^{\pi_{1}}\right)}{\delta_{1}}$, bezw. … $\frac{\varphi\left(p_{s}^{\pi_{s}}\right)}{\delta_{s}}$ ein Basissystem der Gruppe $\Gamma_{\delta}$ constituieren, wird man ohne Weiteres die auf das vollständige Restsystem bezugnehmenden elementaren Sätze über binomische Congruenzen auch auf das System $\Gamma_{\delta}$ ausdebnen können. Der Versuch, dasselbe mit der Theorie der allgemeinen Congruenzen durchzuführen, wird hingegen daran scheitern, dass die Summe zweier $\delta^{\text {ter }}$ Reste durchaus nicht wieder ein solcher zu sein braucht. Doch soll dieser Gegenstand hier nicht weiter ausgeführt werden, vielmehr soll im Folgenden die Theorie der quadratischen Reste, so weit dies ohne Mühe möglich ist, für die $\delta^{\text {ten }}$ Potenzreste entwickelt werden.

Den quadratischen Resten des vollständigen mit $m$ theilerfremden Restsystemes entsprechen innerhalb der Gruppe $\Gamma_{\delta}$ die $(2 \delta)^{\text {ten }}$ Potenzreste, d. h. die Untergruppe $\Gamma_{2 \delta}$. Man hat, nachdem für den Modul $m$ die ungerade Primzahl $p$ und für ò ein Theiler von $\overline{p-1}$ (vergl. \$. 3 der vorigen $\mathrm{Nr}$.) angenommen worden ist, 2 Fälle zu unterscheiden:

1. Der Grad $\frac{p-1}{\delta}$ der Gruppe $\Gamma_{\delta}$ ist eine ungerade Zahl $2 \mu+1$ oder die Primzahl $p$ hat die Form $2 \mu \delta+\delta+1$. 
Dann existiert nach dem Satze (2) 1. c. überhaupt kein Unterschied zwischen $\delta^{\text {ten }}$ und $\left(2 \delta^{\text {ten }}\right.$ Resten. Dieser Fall, bei dem nebenbei bemerkt, die negative Einheit der Congruenz

$$
(-1)^{\frac{p-1}{\delta}} \equiv g^{\frac{\delta}{2} \frac{p-1}{\delta}}(\bmod p)
$$

entsprechend der Klasse $\left(\frac{\delta}{2}\right)$ im Schema $\left(\mathfrak{A}_{\delta, 1}\right)$ 1. c. angehört, gibt somit zu keinen weiteren Betrachtungen Anlass.

2. Die Zahl $\frac{p-1}{\delta}$ ist gerade; der Modul $p$ besitzt also die Form $2 \mu \delta+1$.

Dann gibt es innerhalb der Gruppe $\Gamma_{\delta}$, welcher auch die negative Einheit angehört, ebensoviele $(2 \delta)^{\text {te }}$ Reste, wie Nichtreste. Denn der Grad der Gruppe $\Gamma_{2 \delta}$ beträgt $\frac{p-1}{2 \delta}$. Beide Systeme bleiben nach Multiplication aller ihrer Elemente mit einem Rest ungeändert, vertauschen sich jedoch gegenseitig, wenn man alle Zahlen von $\Gamma_{\delta}$ mit einem Nichtrest multipliciert. Satz:

Dem Euler'schen Kriterium entspricht hier der folgende

Je nachdem die Congruenz

$$
a^{\frac{p-1}{2 \delta}} \equiv-1, \text { oder } \equiv-1 \quad(\bmod p)
$$

besteht, ist der $\delta^{\text {te }}$ Rest $a$ zugleich $(2 \hat{o})^{\text {ter }}$ Rest oder nicht.

Ferner werden, weil die negative Einheit $\delta^{\text {ter }}$ Rest ist, die Zahlen von $\Gamma_{\delta}$, sobald man sie kleiner als $p$ annimmt, ihrer Größe nach geordnet, eine Reihe von der Form

$$
a_{1}, a_{2}, \ldots, a_{\frac{p-1}{2 \delta}}, \overline{p-a}_{\frac{p-1}{2 \delta}}, \ldots, \overline{p-a}_{1}
$$

bilden, wobei der Rest $a_{\frac{p-1}{2 \delta}}$ kleiner als $\frac{p}{2}$ sein wird, so dass sich die $(2 \delta)^{\text {ten }}$ Reste durch die Quadrate $a_{1}^{2}, a_{2}^{2}, \ldots, a_{\frac{p-1}{2 \delta}}^{2}$ darstellen lassen werden.

Auch das Gauss'sche Lemma lässt sich auf die Gruppe $\Gamma_{\delta}$ vollständig übertragen:

Werden die kleinsten ihrem Betrage nach den Wert $\frac{p}{2}$ nicht übertreffenden positiven und negativen Reste der $\delta^{\text {ten }}$ Potenzen

$$
a a_{1}, a a_{2}, \ldots, a a_{\frac{p-1}{2 \delta}}
$$


der Reibe nach mit

$$
r_{1}, r_{2}, \ldots, r_{\frac{p-1}{2 \delta}}
$$

bezeichnet, so stimmt diese Reihe, wenn das Zeichen von einem jeden ihrer negativen Elemente geändert worden ist, vollstiindig mit dem Systeme

$$
a_{1}, a_{2}, \ldots, a_{\frac{p-1}{2 \delta}}
$$

überein. Denn die Beziehung $r_{j} \equiv \pm r_{j}$ zöge die Congruenz $a a_{j} \equiv \pm a a_{j^{\prime}}(\bmod p)$ d. h. die Gleichung $a_{j}=\left\{\begin{array}{l}a_{j^{\prime}} \\ p-a_{j^{\prime}}\end{array}\right.$ nach sich.

Es folgt also aus der Congruenz

$$
\prod_{i=1}^{j=\frac{p-1}{2 \delta}} a_{j} \equiv \prod_{j=1}^{j=\frac{p-1}{2 \delta}} r_{j}(\bmod p)
$$

nach Kürzung mit dem Producte II $a_{j}$ die Beziehung

$$
a^{\frac{p-1}{2 \delta}} \equiv(-1)^{k} \quad(\bmod p)
$$

wenn unter $k$ die Anzahl der negativen Zahlen der Reihe (I) verstanden wird.

Addiert man die Gleichungen

$$
a a_{j}=\left[\frac{a a_{j}}{p}\right] p+r_{j}^{\prime} \quad\left(j=1,2, \ldots, \frac{p-1}{2 \delta}\right),
$$

in denen der Rest $r_{j}^{\prime}$ natürlich, je nachdem $r_{j}$ das positive oder negative Zeichen besitzt, gleich $r_{j}$ oder $p+r_{j}$ ist, so erhält man die Beziehung

$$
a \sum_{j=1}^{\frac{p-1}{2 \delta}} a_{j}=p \sum_{j=1}^{\frac{p-1}{2 \delta}}\left[\frac{a a_{j}}{p}\right]+k p+\sum_{j=1}^{\frac{p-1}{2 \delta}} r_{j} .
$$

Nimmt man zunächst an, dass $2 \delta^{\text {ter }}$ Rest ist, und setzt man in der gefundenen Gleichung für $a$ die Zahl 2, für $\delta$ aber eine ungerade Zahl, so bekommt man die Relation

$$
\sum_{j=1}^{\frac{p-1}{28}} a_{j} \equiv k \quad(\bmod 2)
$$


aus welcher sich unter Berücksichtigung des Umstandes, dass die Zahl 2 bei den gemachten Annahmen gleichzeitig quadratischer und $(2 \delta)^{\text {ter }}$ Rest ist oder nicht, der folgende Satz auf Grund der bekannten Gleichung $\left(\frac{p}{2}\right)=(-1)^{\frac{p^{2}-1}{8}}$ ergibt:

Die Summeder positiven ${ }^{\text {ten }}$ Reste, welche kleiner als $\frac{p}{2}$ sind, ist gleichzeitig mit $\frac{p^{2}-1}{8} \mathrm{ge}-$ rade oder ungerade, wenn $\delta$ ungerade ist und die Zah1 2 sich unter den $\delta^{\text {ten }}$ Resten vorfindet.

Natürlich wird die Anzahl der $\delta^{\text {ten }}$ Reste, welche kleiner als $\frac{p}{4}$ sind, durch die Differenz $\frac{p-1}{2 \delta}-k$ bestimmt, falls 2 unter den $\delta^{\text {ten }}$ Resten vorkommt und $k$ auf die Zahl 2 bezogen wird.

Aus der Gleichung (II) kann man ferner, wenn man $a$ ungerade voraussetzt, ohne Weiteres die Congruenz

$$
k \equiv \sum_{j=1}^{\frac{p-1}{2 \delta}}\left[\frac{a a_{j}}{p}\right]
$$

herleiten, welche eine bekannte Relation erweitert.

Wie man sieht, herrscht bis zu diesem Punkte vollständige Analogie mit der Theorie der quadratischen Reste. Während aber in dem Falle $\delta=1$ die Formel (III) die Grundlage zu einer Reihe von sehr einfachen Beweisen für das Reciprocitätsgesetz bildet, scheinen die Verhältnisse im allgemeinen Falle sich verwickelter zu gestalten, worauf jedoch hier nicht weiter eingegangen werden soll.

$$
\text { §. } 2 .
$$

Fügt man zu den Elementen des Schemas $\left(\mathfrak{A}_{\delta, 1}\right)$ im $\S .3$ der vorigen Nr. alle positiven Multipla von $p$ hinzu und setzt man die erhaltenen Zahlen der Größse nach geordnet in die entsprechenden Klassen, so hat man das Gebiet aller natürlichen dureh $p$ nicht theilbaren Zahlen in $\delta$ Klassen eingetheilt. Es soll im Folgenden die Klasse $(0)$, d. $\mathrm{h}$. die Gesammtheit der modulo $p$ congruenten und incongruenten $\delta^{\text {ten }}$ Potenzreste

$$
a_{1}=1, a_{2}, \ldots, a_{\frac{p-1}{\delta}}, a_{\frac{p-1}{\delta}+1}=p+1, a_{\frac{p-1}{\delta}+2}=p+a_{2}, \ldots
$$

in Bezug auf einige Theilbarkeitsfragen untersucht werden.

Man erkennt zunächst, dass sich zwar das Product je zweier Individuen ron (0) wieder in der Reihe $(0)$ vorfindet, dass dies aber keineswegs mit jedem Factor irgend einer Zahl dieses Systemes der Fall $z u$ sein braucht. Man hat zwischen Primzahlen, die $\delta^{\text {te }}$ 
Reste theilen und selbst $\delta^{\text {te }}$ Reste sind, and zwischen Primzahlen, die zwar $\delta^{\text {te }}$ Reste theilen, aber anderen Klassen vọn $\left(\mathfrak{A}_{\delta, 1}\right)$ angehören, zu unterscheiden. Bliebe man bei den innerhalb des Systemes ( 0 ) unzerlegbaren Zahlen als Elementarzahlen stehen, so würde die Erscheinung auftreten, dass gewisse Individuen von (0) sich auf mehrere Arten in solche unzerlegbare Elemente spalten lieben.

Zu einer jeden Klasse $(i)$ des Schemas $\left(\mathfrak{A}_{\delta, 1}\right)$ gehört nämlich eine und nur eine Klasse $(\delta-i)$ der Art, dass das Product irgend eines Individuums der einen in irgend ein Individuum der zweiten Klasse einen $\delta^{\text {ten }}$ Rest als Resultat ergibt. (Vergl. Nr. 6 im II. Abschn.) Es soll dann eine Klasse kurz „die ergänzende Klasse" der anderen genannt werden.

Gehören nun die beiden Primzahlen $q$ und $q^{\prime}$ der nämlichen Klasse, die Primzahlen $r$ und $r^{\prime}$ aber der diese ergänzenden Klasse an, so sind die Producte $q r$ und $q^{\prime} r^{\prime}$ beide $\delta^{\text {to }}$ Reste und zwar im Gebiete (0) nicht weiter zerlegbar. Das Gleiche gilt jedoch auch von den beiden Zahlen $q r^{\prime}$ und $q^{\prime} r$. Man hat also den Rest $\left(q q^{\prime} r r^{\prime}\right)$ auf doppelte Weise in im Gebiete (0) nicht weiter spaltbare Factoren zerlegt:

$$
\left(q q^{\prime} r r^{\prime}\right)=(q r)\left(q^{\prime} r^{\prime}\right)=\left(q r^{\prime}\right)\left(q^{\prime} r\right) \text {. }
$$

Um diese Erscheinung, welche bekanntlich $\mathrm{Kummer}$ in einem anderen Gebiete zur Einführung der Idealzahlen veranlasst hat, zu beseitigen, braucht man hier natürlich nur alle anderen Primzahlen, also auch alle anderen Klassen neben der Klasse (0) zu beriicksichtigen.

Speciell kommt bei Theilbarkeitsfragen der Klasse (0) das Verhältnis von zwei sich ergänzenden Klassen in Betracht. So erkennt man ohne Weiteres, dass die Reihe

$$
k a_{1}^{\left(i_{k}\right)}, k a_{2}^{\left(i_{k}\right)}, k a_{3}^{\left(i_{k}\right)}, \ldots
$$

genau die Zahlen des Systemes (0), welche durch $k$ theilbar sind, darstellt, wenn, wie diess auch immer im Folgenden geschehen soll, unter $i_{k}$ der Zeiger derjenigen Klasse verstanden wird, welche die das Indjviduum $k$ enthaltende Klasse ergänzt. Mithin wird die Anzabl derjenigen $\delta^{\text {ten }}$ Reste, die durch $k$ theilbar sind and den Wert $\alpha$ nicht iubertreffen, dureh die Anzahl

$$
\left[\frac{\alpha}{\bar{k}}\right]_{i_{\bar{k}}}
$$

derjenigen Individuen der Klasse $\left(i_{k}\right)$, welche nicht größer als $\frac{a}{b}$ sind, bestimmt werden. 
Die hier auftretende Anzahl $[\alpha]$; der den Wert $\alpha$ nicht übertreffenden Individuen der Klasse $(j)$ ist mit der gröbten ganzen noch in $\alpha$ enthaltenen Zahl $[\alpha]$ verwandt.

Es ist zunächst klar, dass die Anzahl $[\alpha]_{0}$ unter der speciellen Annahme $\delta=1$ mit der Differenz $[\alpha]-\left[\frac{a}{p}\right]$, also im Gebiete $1,2, \ldots, \overline{p-1}$ mit $[a]$ selbst übereinstimmt, während im allgemeinen Falle die Gleichung

$$
\sum_{j=0}^{\delta-1}[\alpha]_{j}=[\alpha]-\left[\frac{\alpha}{p}\right]
$$

besteht. Der Subtrahend $\left[\frac{\alpha}{p}\right]$ tritt auf der rechten Seite dieser Gleichung aus dem Grunde auf, weil auf der linken die Vielfachen von $p$ nicht gezählt werden.

Ferner erkennt man ohne Weiteres, das die Anzahl $\left[\frac{\alpha}{h k}\right]_{i_{h k}}$ der $\delta^{\text {tan }}$ Reste, die durch das Product $h k$ theilbar sind und den Wert a nicht überschreiten, auch dadurch gewonnen werden kann, dass man abzählt, wie viele Individuen der Reihe

$$
a_{1}^{\left(i_{i}\right)}, a_{2}^{\left(i_{k_{2}}\right)}, \ldots, a_{s}^{\left(i_{k}\right)} \quad\left(s=\left[\frac{a}{k}\right]_{i_{k}}\right)
$$

durch $h$ theilbar sind. Man erhält auf diese Art die Gleichung.

$$
\left[\frac{a}{h k}\right]_{i_{i k k}}=\left[\frac{a_{s}^{\left(i_{k}\right)}}{h}\right]_{i_{h k}},
$$

welche unter Berücksichtigung der Vertauschbarkeit von $h$ und $k$ den folgenden Satz ergibt:

$$
Z_{\text {wischen den drei Werten }} \frac{\alpha}{h \bar{k}}, \frac{a_{s}^{\left(i_{k}\right)}}{h}\left(s=\left[\begin{array}{l}
\alpha \\
\bar{k}
\end{array}\right]_{i_{k}}\right)
$$

$$
\text { und } \frac{a_{s^{\prime}}^{\left(i_{h}\right)}}{k}\left(s^{\prime}=\left[\frac{a}{h}\right]_{i_{h}}\right) \text { liegtkeine Zahl der Klasse } i_{h k} \text {. }
$$

Die Relation (I) geht unter der Annahme $\delta=1$ in die bekannte Gleichung

$$
\left[\frac{\alpha}{h \bar{k}}\right]=\left[\frac{\left[\frac{a}{k}\right]}{h}\right]
$$

über. 
Es bedart keiner weiteren Auseinandersetzung, dass dis höchste noch in dem Producte $a_{1} . a_{2} \ldots a_{s}$ enthaltene Potenz der Primzahl $q$ durch die Summe

bestimmt wird.

$$
\left[\frac{a_{r}}{q}\right]_{i_{q}}+\left[\frac{\alpha_{r}}{q^{2}}\right]_{i_{q^{2}}}+\cdots
$$

Weiters findet man anch hier genau, wie im gewöhnlichen Falle, mit Hilfe der Methode des Ausscheidens und Hinzufügens für die Anzahl derjenigen Individuen von (0), welche nicht größer als $\alpha$ und durch keine der untereinander theilerfremden Zahlen $n_{1}, \ldots, n_{t}$ theilbar sind, den Ausdruck

$$
[\alpha]_{0}-\sum_{n}\left[\frac{\alpha}{n}\right]_{i_{n}}+\sum_{n n^{\prime}}\left[\frac{\alpha}{n n^{\prime}}\right]_{i_{n n^{\prime}}}-\cdots
$$

Bedeutet die Function $\varphi_{\lambda}(m)$ die Anzahl derjenigen Individuen der Klasse $(\lambda)$, welche nicht größer als $m$ und mit $m$ theilerfremd sind, so besteht die Gleichung

$$
\sum_{\tau} i_{i}\left(\frac{\alpha}{\tau}\right)=[a]_{0}
$$

in der $\tau$ alle Theiler der ganzen Zahl $\alpha$ zu durchlaufen hat. Denn jedes Individuum $a_{j}$ hat mit $\alpha$ einen bestimmten gröften gemeinsamen Theiler $\tau$ und die Anzahl der Individuen von (0) dieser Eigenschaft beträgt $s_{i}\left(\frac{a}{\tau}\right)$.

Da zwischen je 2 aufeinanderfolgenden Vielfachen von $p$ genau $\frac{p--1}{\partial}$ Individuen einer jeden Klasse gelegen sind, führt die Gleichung

$$
[\alpha]_{j}=\left[\frac{\alpha}{p}\right] \frac{p-1}{o}+[r]_{j} \quad(0 \leqq r<p)
$$

die Anzahl $[\alpha]_{j}$, bei der die Zahl $\alpha$ nicht kleiner als $p$ ist, auf den Fall $[r]_{j}$, zurück, wo der bei der Division von $\alpha$ durch $p$ auftretende Rest $r$ kleiner als $p$ ist.

Wird mit $f(u)$ eine willkürliche Function von $\mu$, mit $\varepsilon(\mu)$ aber die bekannte zahlentheoretische Function bezeichnet, welche 1 oder 0 bedeutet, jenachdem $\mu \geq 1$ oder $<1$ ist, so besteht unter der Annahme $\alpha<p$ die Gleichung

$$
\sum_{j=1}^{a}\left[\frac{\alpha}{j}\right]_{i j} f(j)=\sum_{j=1}^{[a]_{0}}\left\{\varepsilon\left(\frac{a}{a_{j}}\right) \sum_{a} f(d)\right\},
$$


in der $d$ alle Theiler von $a_{j}$ zu durchlauten hat. Anf der linken Seite der Gleichung (III) lässt sich nämlich die Zahl $\left[\frac{a}{j}\right]_{i_{3}}$ durch die Summe

$$
\sum_{\sigma=a_{1}^{(i j)}, a_{2}^{(i, j)} \ldots} \varepsilon\left(\frac{a}{j \sigma}\right)
$$

ersetzen; das Product $j . \sigma$ gibt stets einen $\delta^{\text {ten }}$ Rest, so dass man die auftretende Doppelsumme nach den $\delta^{\text {ten }}$ Resten ordnen kann, wodurch man den Ausdruck auf der rechten Seite der Relation (III) erhält.

Es mögen insbesondere die folgenden drei speciellen Fälle der Gleichung (III) hervorgehoben werden:

Setzt man erstens für $f(j)$ die bekannte zahlentheoretische Function $\mu(j)$, so erhält man die Gleichung

$$
\sum_{j=1}^{a}\left[\frac{a}{j}\right]_{i j} \mu(j)=1
$$

die sich auch ohne Weiteres aus der Bedeutung des Ausdruckes (II) ergibt.

Nimmt man zweitens für $f(j)$ stets die Einheit, so erhält man für die Summe der Anzahlen aller Theiler eines jeden $\delta^{\text {ten }}$ Restes, der den Wert a nicht übersteigt, den Ausdruck

$$
\sum_{i=1}^{\alpha}\left[\frac{a}{j}\right]_{i_{j}} .
$$

Endlich ergibt die Specialisierung $f(j)=\varphi(j)$ die Relation

$$
\sum_{j=1}^{a}\left[\begin{array}{l}
\alpha \\
j
\end{array}\right]_{i j} \rho(j)=\sum_{j=1}^{[\alpha]_{0}} a_{j},
$$

aus welcher man mittelst der Annahme $\alpha=\overline{p-1}$ im Falle, dass $\frac{p-1}{\delta}$ gerade, also $(-1) \delta^{\text {ter }}$ Rest ist, die Gleichung

$$
\sum_{j=1}^{p-1}\left[\frac{p-1}{j}\right]_{i_{j}} \rho(j)=\frac{p(p-1)}{2 \delta}
$$

herleitet. (Vergl. \$. 3 in Nr. 3.)

Schlieblich sei noch bemerkt, dass die im Vorstehenden für die $\delta^{\text {ten }}$ Potenzreste entwickelten Sätze für jede Klasse des Schemas $\left(\mathfrak{A}_{\delta, 1}\right)$ gelten, wenn nur der Begriff der „ergänzenden Klasse" in entsprechender Weise modificiert wird. 
Notiz über die Zerlegung der ganzen Zahlen. Von Karl Glösel in Bielitz.

Tch werde in den folgenden Zeilen einen verhältnismäßig sehr einfachen Ausdruck für $C_{5}(\sigma)$ ableiten.

Die in meiner Arbeit über die Zerlegung der Zahlen ${ }^{1}$ ) angegebene Rolation

$$
S=C_{3}^{1}(x)+C_{3}^{1}(x-5)+C_{3}^{1}(x-10)+\cdots=\left\{\frac{\left(\begin{array}{c}
x+4 \\
4
\end{array}\right)}{5}\right\}-C_{3}^{2}(x+5)
$$

lässt sich, wie man leicht findet, auf die Gestalt

$$
\begin{gathered}
S=\frac{1}{720}\left((x+4)(x+2)(x-2)(x-4)-144\left(\left[\frac{x-5}{5}\right]-\left[\frac{x+4}{5}\right]\right)+\right. \\
\left.\quad+80\left(\left[\frac{x+6}{3}\right]-\left[\frac{x+5}{3}\right]\right)-45\left(\left[\frac{x+7}{2}\right]-\left[\frac{x+6}{2}\right]\right)\right)
\end{gathered}
$$

bringen, woraus, da der bei der Division von

$$
144\left(\left[\frac{x+5}{5}\right]-\left[\frac{x+4}{5}\right]\right)-80\left(\left[\frac{x+6}{3}\right]-\left[\frac{x+5}{3}\right]\right)+45\left(\left[\frac{x+7}{2}\right]-\left[\frac{x+6}{2}\right]\right)
$$

durch 720 verbleibende Rest absolut genommen kleiner als $\frac{1}{2}$ ist, folgt

$$
S=\left\{\frac{(x+4)(x+2)(x-2)(x-4)}{720}\right\}
$$

Nun bat man mach den von mir a. a. O. aufgestellten Formeln

$$
\begin{gathered}
C_{4}(\sigma)=C_{3}^{1}\left(\left[\frac{\sigma+2}{2}\right]\right)+C_{3}^{1}\left(\left[\frac{\sigma-1}{2}\right]\right) \\
\lambda=\left[\frac{\sigma-\left(\begin{array}{c}
r+1 \\
2
\end{array}\right)}{r}\right] \\
C_{r}(\sigma)=\sum_{\lambda=1} C_{r-1}(\sigma-\lambda r)
\end{gathered}
$$

die Gleichung

$$
\begin{aligned}
& \left.C_{\sigma}(\sigma)=C_{3}^{1}\left(\frac{\sigma-3}{2}\right]\right)+C_{3}^{1}\left(\left[\frac{\sigma-13}{2}\right)\right]+C_{3}^{1}\left(\left[\frac{\sigma-23}{2}\right]\right)+\cdots+C_{3}^{1}\left(\left[\frac{\sigma-8}{2}\right]\right)+ \\
& \quad+C_{3}^{1}\left(\left[\frac{\sigma-18}{2}\right]\right)+C_{3}^{1}\left(\left[\frac{\sigma-28}{2}\right]\right)+\cdots+C_{3}^{1}\left(\left[\frac{\sigma-6}{2}\right]\right)+C_{3}^{1}\left(\left[\frac{\sigma-16}{2}\right]\right)+ \\
& +C_{3}^{1}\left(\left[\frac{\sigma-26}{2}\right]\right)+\cdots+C_{3}^{1}\left(\left[\frac{\sigma-11}{2}\right]\right)+C_{3}^{1}\left(\left[\frac{\sigma-21}{2}\right]\right)+C_{3}^{1}\left(\left[\frac{\sigma-31}{2}\right]\right)+\cdots
\end{aligned}
$$

und daher erhält man unter Benützung von (1) die Beziehung:

$$
\begin{aligned}
& C_{5}(\sigma)=\left\{\frac{\left\{\left(\left[\frac{\sigma-3}{2}\right]\right)^{2}-16\right)\left(\left(\left[\frac{\sigma-3}{2}\right]\right)^{2}-4\right)}{720}\right\}+\left\{\frac{\left.\int\left(\left[\frac{\sigma-6}{2}\right]\right)^{2}-16\right)\left(\left(\left[\frac{\sigma-6}{2}\right]\right)^{2}-4\right)}{720}\right\}+ \\
& \left.+\left\{\frac{\left(\left(\left[\frac{\sigma-8}{2}\right]\right)^{2}-16\right)\left(\left(\left[\frac{\sigma-8}{2}\right]\right)^{2}-4\right)}{720}\right\}+\left\{\left(\left[\frac{[-11]}{2}\right]\right)^{2}-16\right)\left(\left(\left[\frac{\sigma-11}{2}\right]\right)^{2}-4\right)\right\}
\end{aligned}
$$

1) Diese Monatshefte, VII. Jahrg., S. $130 \mathrm{ff}$. 